\title{
Regularization around a generic codimension one fold-fold singularity
}

\author{
Carles Bonet-Reves*, Juliana Larrosa ${ }^{\dagger}$, Tere M-Seara*
}

\begin{abstract}
This paper is devoted to study the generic fold-fold singularity of Filippov systems on the plane, its unfoldings and its Sotomayor-Teixeira regularization. We work with general Filippov systems and provide the bifurcation diagrams of the fold-fold singularity and their unfoldings, proving that, under some generic conditions, is a codimension one embedded submanifold of the set of all Filippov systems. The regularization of this singularity is studied and its bifurcation diagram is shown. In the visible-invisible case, the use of geometric singular perturbation theory has been useful to give the complete diagram of the unfolding, specially the appearance and disappearance of periodic orbits that are not present in the Filippov vector field. In the case of a linear regularization, we prove that the regularized system is equivalent to a general slow-fast system studied by Krupa and Szmolyan KS01b.
\end{abstract}

Keywords: Non-smooth systems; Regularization; Bifurcations; Melnikov Method; Singular perturbation theory.

\section{Introduction}

In this paper, derived from the thesis [Lar15, we study the generic fold-fold singularity of Filippov systems on the plane, its unfoldings and its regularization, more concretely, its SotomayorTeixeira regularization [ST98].

The first part of the paper is devoted to study the fold-fold singularity. This singularity has been studied in [KRG03 and GST11 by considering some simple normal forms for the Filippov vector fields and their unfoldings, and also in the original book of Filippov [Fil03]. A systematic study of the set of structurally stable Filippov vector fields was done in [GST11] but, besides the previously mentioned works, which study normal forms, there does not exist a rigorous approach to the codimension one singularities. Our goal, realized in Theorem 2.6, is to work with general Filippov systems and provide the bifurcation diagrams of these singularities and their unfoldings, proving that the set of the fold-fold singularities, under some generic conditions, is a codimension one embedded submanifold of $\Xi_{0}$, the set of structurally stable Filippov systems.

The second part of the paper is dedicated to study the regularization of the unfoldings of the foldfold singularity and is a natural continuation of the paper [RS14], where Filippov vector fields near a fold-regular point were considered. It is known BST06; TS12 that, under general conditions, in the so-called sliding and escaping regions, the regularized system has a normally hyperbolic invariant manifold, attracting near the sliding region and repelling near the escaping one. Furthermore, the flow of the regularized vector field reduced to this invariant manifold approaches to the Filippov flow. In [RS14], these results were extended to visible tangency points, using asymptotic methods following [MR80]. The work [KH15a extended these results to $\mathbb{R}^{3}$ by use of blow-up methods.

The results in this work are mostly given in [Lar15], therefore the cumbersome computations are referred to it.

\footnotetext{
*BGSMATH, Dpt. Matemàtiques, Universitat Politècnica de Catalunya, Diagonal 647, 08028 Barcelona,Spain.

${ }^{\dagger}$ Departamento de Matemática, Universidade Federal de Santa Maria, Avenida Roraima 1000, 97195-000 Santa Maria, RS, Brasil.
} 
During the period of time of writing this paper, the work KH15b, where the authors study this problem, came out. In $\mathrm{KH} 15 \mathrm{~b}$ the authors perform some changes of variables to simplify the system and then study the normal form obtained using blow-up methods and analyzing it in different charts (variables). Their analytic approach completely characterizes the existence and the attracting/repelling character of the equilibrium points showing that in some relevant cases, there is a curve in the parameter plane where the equilibrium of the system has a Hopf bifurcation They also show that the (sub/super critical) character of the Hopf bifurcation depends on the considered Filippov vector fields but also on the regularization function. In fact, in formula (7.15) of that paper, the authors give an explicit formula for the Lyapunov coefficient at the Hopf bifurcation for the normal form system. They also study the appearance and character of the family of periodic orbits at the Hopf bifurcation and their evolution. In the invisible-invisible case, they succeed in describing the family as a smooth family of locally unique periodic orbits, that, in some cases, can undergo a saddle-node bifurcation. In the visible-invisible case, they prove the existence of a curve in the parameter plane where a Maximal Canard occurs. Moreover, they prove the existence of a family of locally unique "big" periodic orbits for parameters (exponentially) close to the Canard curve. The authors conjecture that the "small" curves near the Hopf bifurcation and the "big" curves near the Canard curve belong to the same smooth family of locally unique periodic orbits.

The approach in our paper is mainly topological providing some new and slightly different results which complement the ones obtained in $\mid \mathrm{KH} 15 \mathrm{~b}]$; one major goal is to give results directly checkable in a given system, for this reason we work in the original variables of the system, and we present its possible phase portraits. We use topological methods to get the generic conditions which determine the phase portrait in terms of some intrinsic and explicit quantities that can be computed directly from the original system. For this reason, although KH15b already computed the values of the Hopf and Canard curves, we can not rely in their formulas (7.14) and (6.22) because they are only valid for systems in normal form and we have done these computations for general vector fields in Propositions 4.6, 3.7.

We now present these different results and the new ones presented in this paper.

In the visible-invisible case we prove the existence of a periodic orbit for any value of the parameters between the Canard and the Hopf curves in theorems 4.7 and 4.8 whose stability depends on the relative position of these curves. We stress that this result is purely topological and follows the same kind of argument used in the invisible-invisible case to prove the existence of a stable periodic orbit for any value of the parameters after the Hopf bifurcation curve in Proposition 4.2 Furthermore, in proposition 4.11 we give precise information about the region of existence of the "big" periodic orbit which appears close to the Canard curve, establishing that it exists before the Canard curve when it is unstable and after the Canard curve when it is stable using again topological reasonings. Moreover, the stability of this "big" periodic orbit is studied and we show that, analogously to what happens at the Hopf bifurcation, it depends on the considered Filippov vector field but also on the regularization function as formula (4.13) proves.

This topological approach does not answer the conjecture of [KH15b] but it gives a precise information about the domain of existence of the periodic orbits in the visible-invisible case and their possible saddle-node bifurcations.

Moreover, in the visible-invisible case when the transition function is linear, we present some new results about the position of the curve in the parameter plane where the maximal Canard exists. We also provide the complete bifurcation diagram of the regularized system.

In KH15b a Melnikov-based argument introduced in [KS01b was used to continue the small periodic orbits arising at the Hopf bifurcation. In section 5 we develop the Melnikov method to compute periodic orbits and in Proposition 5.1 we study the properties of the Melnikov function and show how this function can be used, as an alternative to the Lyapunov coefficient, to detect the subcritical/supercritical character at the Hopf bifurcation in a given system. We also give conditions on this function that guarantee global unicity of the periodic orbits both in the visiblevisible or the visible-invisible cases (see Proposition 4.2 and Theorem 4.3), and we show that it can be used to compute the saddle-node bifurcations in concrete examples (see Proposition 4.5). 
Now we explain the contain of the paper. We consider a Filippov vector field $Z=(X, Y)$ having a fold-fold point, that we assume being at the origin $(x, y)=\mathbf{0}$, we take $Z^{\alpha}$ its unfolding, were $\alpha$ is the unfolding parameter, and its regularization $Z_{\varepsilon}^{\alpha}$ (see Equation (3.1)], where $\varepsilon$ is the regularization parameter. Our goal is to see if the dynamics of $Z_{\varepsilon}^{\alpha}$ is equivalent, from a topological point of view, to the one of $Z^{\alpha}$. The results are different depending on the fold-fold type, which can be visible-visible, invisible-invisible or visible-invisible.

As can be expected, the behavior of the regularized system $Z_{\varepsilon}^{\alpha}$ is similar to the one of $Z^{\alpha}$ if we fix $\alpha \neq 0$ and consider $\varepsilon$ small enough; if $Z^{\alpha}$ has a sliding zone in its switching surface and the sliding vector field has a pseudo equilibrium $Q(\alpha)$, then the regularized vector field $Z_{\varepsilon}^{\alpha}$ has an equilibrium $P(\alpha, \varepsilon)$ of the same type. Both conditions depend on the original vector field $Z=(X, Y)$ satisfying $X^{1} \cdot Y^{1}(\mathbf{0})<0$. Analogously, when $Z^{\alpha}$ has a crossing periodic orbit, the regularized vector field $Z_{\varepsilon}^{\alpha}$ has a periodic orbit of the same type.

In the visible-visible case, both the unfolding $Z^{\alpha}$ and its regularization $Z_{\varepsilon}^{\alpha}$ have the same topological type if $\alpha$ and $\varepsilon$ are small enough: the critical point $P(\alpha, \varepsilon)$ is a saddle point for $Z_{\varepsilon}^{\alpha}$ $\left(Q(\alpha)\right.$ is a pseudo-saddle for $\left.Z^{\alpha}\right)$ and there is no other interesting dynamics near it.

The invisible-invisible case is more involved. In this case, the fold-fold is the so-called pseudofocus case in the language of Filippov systems [KRG03, and its attracting or repelling character can be checked studying the return map around it (c.f Tei81]). First, we see that the character of the critical point $P(0, \varepsilon)$ of the regularization $Z_{\varepsilon}$ is independent of the character of the fold-fold point: $P(0, \varepsilon)$ can be a (repelling or attracting) focus or a center. One understands better the dynamics when one considers the regularization of the unfoldings $Z_{\varepsilon}^{\alpha}$. It is known that $Z^{\alpha}$ has a pseudo-node $Q(\alpha)$. We see that $P(\alpha, \varepsilon)$ is a node with the same character as $Q(\alpha)$ for fixed $\alpha \neq 0$ and $\varepsilon$ small enough. We also find a curve $\mathcal{D}$ in the parameter plane of the form $\varepsilon=C \alpha^{2}+\mathcal{O}\left(\alpha^{3}\right)$ where the critical point $P(\alpha, \varepsilon)$ becomes a focus and another curve $\mathcal{H}$ of the form $\alpha=\delta_{\mathcal{H}} \varepsilon+\mathcal{O}\left(\varepsilon^{2}\right)$ where there is a Hopf bifurcation which creates a periodic orbit $\Delta_{\varepsilon}^{\alpha, *}(*=s, u$ since the orbit can be stable or unstable depending of the character of the Hopf bifurcation). On the other hand, it is well known ([KRG03] $)$ that $Z^{\alpha}$ has a periodic crossing cycle $\Gamma^{\alpha}$ for $\alpha$ at one side of 0 . We can prove that for $\alpha$ and $\varepsilon$ at one side of the Hopf curve, $Z_{\varepsilon}^{\alpha}$ has a periodic orbit $\Gamma_{\varepsilon}^{\alpha, *}$ whose character is the opposite to the one of the critical point. Moreover, for fixed $\alpha$ and $\varepsilon$ small enough a periodic orbit $\Gamma_{\varepsilon}^{\alpha, *}=\Gamma^{\alpha}+\mathcal{O}(\varepsilon)$ exists for the regularization $Z_{\varepsilon}^{\alpha}$. One would expect that the periodic orbit created at the Hopf bifurcation of the regularization $Z_{\varepsilon}^{\alpha}$ increases in size until it becomes $\Gamma_{\varepsilon}^{\alpha, *}$, but this is not always the case. Depending on the attracting/repelling character of the fold-fold given by the return map and sub/supercritical character of the Hopf bifurcation, both periodic orbits can appear in a saddle-node bifurcation of periodic orbits and only the "big one" $\Gamma_{\varepsilon}^{\alpha, *}$ persists and becomes the cycle $\Gamma^{\alpha}$.

In short: the periodic orbit arising from the non-smooth crossing cycle can either "die" at the Hopf bifurcation or coexist with the periodic orbit born at the Hopf bifurcation, and both die in a saddle node bifurcation of periodic orbits. It is important to stress that, as has been already observed in $[\mathrm{KH} 15 \mathrm{~b}$, the character of the Hopf bifurcation depends on the transition function.

The dynamics is richer in the regularization of the visible-invisible fold. The first observation is that the unfoldings of this fold are of different topological behavior depending on an intrinsic quantity of the original Filippov vector field $Z$. In one case, the pseudo equilibrium $Q(\alpha)$ of the unfolding $Z^{\alpha}$ is a saddle point and its dynamics and the one of its regularization $Z_{\varepsilon}^{\alpha}$ are topologically equivalent. This case is similar to the visible-visible fold.

The other case is the one which presents the more interesting dynamics. The pseudo-equilibrium $Q(\alpha)$ of the unfolding $Z^{\alpha}$ is a node and the behavior is similar to the invisible-invisible case. The critical point $P(\alpha, \varepsilon)$ is also a node with the same character as $Q(\alpha)$ for fixed $\alpha \neq 0$ and $\varepsilon$ small enough. It becomes a focus when the parameters cross the parabola $\mathcal{D}$ and suffers a Hopf bifurcation at the curve $\mathcal{H}$.

The most difficult question is to determine what happens with the periodic orbit $\Delta_{\varepsilon}^{\alpha, *}$ that appears at the Hopf bifurcation because there are no periodic orbits in the unfolding $Z^{\alpha}$. In order to understand this phenomenon we have to investigate the slow-fast nature of the regularized vector 
field $Z_{\varepsilon}^{\alpha}$ when written in scaled variables. Using the methods of singular perturbation theory, we have proved that this slow-fast system has a stable Fenichel manifold and an unstable one that coincide along a maximal Canard if the parameters are in a curve $\mathcal{C}$ of the form $\alpha=\delta_{\mathcal{C}} \varepsilon+\mathcal{O}\left(\varepsilon^{3 / 2}\right)$. The existence of this maximal Canard creates a big periodic orbit $\Delta_{\varepsilon}^{\alpha, \mathcal{C}}$ (the so-called Canard explosion phenomenon, see $[\mathrm{KH} 15 \mathrm{~b}]$ ). Then, depending o the character of both periodic orbits, the interaction between this "big one" $\Delta_{\varepsilon}^{\alpha, \mathcal{C}}$ with the "small one" $\Delta_{\varepsilon}^{\alpha, *}$, emerging at the Hopf bifurcation, creates a richer dynamics that makes the orbits disappear when they meet at a different saddlenode bifurcations. Our analysis shows that, analogously to the Hopf bifurcation, the attracting or repelling character of the periodic orbit arising at the Canard also depends on the transition function.

The paper is organized as follows. In section 2, we recall the basic concepts of Filippov vector fields and the intrinsic quantities which characterize the different types of fold-fold. The main result of this section is Theorem 2.6 where we prove that the fold-fold singularity satisfying some generic conditions is a codimension one singularity. The proof of the theorem also gives the dynamics of its versal unfoldings that will be needed in the following sections where we consider the SotomayorTeixeira regularization.

Section 3 considers the regularization $Z_{\varepsilon}^{\alpha}$ of an unfolding of the fold-fold singularity and the slow-fast system (3.5) associated to it. The first part of this section is devoted to studying the critical points of $Z_{\varepsilon}^{\alpha}$ and the second, to studying the critical manifolds of the slow-fast system.

Section 4 gives the dynamics of the regularized vector field. The section is separated in three cases, one for each type of fold. The visible-visible case is the simplest and is studied in subsection 4.1 .

The invisible-invisible case is studied in subsection 4.2. The main results in this section are Proposition 4.2, which prove the existence of the periodic orbit at one side of the Hopf bifurcation, independently of the nature of this bifurcation, and also guarantees that this orbit is near the crossing cycle of the non-smooth system when $\alpha$ is fixed and $\varepsilon$ is small enough. Theorems 4.3 and 4.4 provide a complete description of the evolution of the dynamics when the parameters $(\alpha, \varepsilon)$ move around the origin. In particular, we observe that the regularized system may have saddlenode bifurcations of periodic orbits which do not exist in the unfolding $Z^{\alpha}$. Following the ideas of [KS01b] (see also [KH15b]), we also present in Proposition 4.5 some results about the use of a suitable Melnikov function to give the local uniqueness of the periodic orbits and to compute the value of the parameters where the saddle node bifurcation takes place, if it exists. We conclude this section showing some examples that illustrate the behavior described in these results.

The visible-invisible case is studied in subsection 4.3 and presents two different behaviors. In subsubsection 4.3.3 we study the case that the critical point is a saddle, which is similar to the visible-visible case. In subsubsection 4.3.1 we analyze the case where the critical point is first a node, then becomes a focus and finally undergoes a Hopf bifurcation. In Proposition 4.6 we prove the existence of a maximal Canard in a curve of the parameter plane $(\alpha, \varepsilon)$. Theorems 4.7 and 4.8 provide the phase portrait of the system, including the behavior of the periodic orbits, depending on the position of the Canard and the Hopf curves, as well as on the nature of the Hopf bifurcation. In Theorem 4.10 we see that system (3.5) can be transformed into the general slow-fast system studied in KS01b by changes of variables if the transition function $\varphi$ is linear. This completely determines the position of the Canard curve depending on the sign of the Lyapunov coefficient at the Hopf bifurcation. Therefore, in the linear case, only the results of Theorem 4.7 are possible. These theorems are complemented with Proposition 4.11, where we give a formula for the coefficient which determines the stable/unstable character of the periodic orbit near the Canard and we show how the periodic orbit disappears in a so-called Canard explosion or in a saddle node bifurcation depending on its stability. We conclude this section with some examples which illustrate the most interesting behaviors described in the section.

The aim of section 5 is to recover the periodic orbits of moderate size of the system when $\alpha=\delta \varepsilon$ using classical perturbation theory after some scaling of the variable $x=\sqrt{\varepsilon} u$. This provides the so-called Melnikov function $M(v ; \delta)$, whose simple zeros give locally unique periodic orbits of the 
system. Even if the existence of periodic orbits in the invisible-invisible case and in the visibleinvisible case of focus type are obtained without using this function, it is useful to derive their uniqueness and to give a computable method to obtain the value of the parameters where the saddle-node bifurcations occur. For this reason, we think is worthwhile to dedicate a short section to this function, its properties and recover the results about periodic orbits in theorems 4.3 . 4.4 . 4.7 and 4.8 .

Finally, we postpone to the Appendix the more technical proofs of Proposition 4.6 in subsection 7.2, the proof of Theorem 4.10 in 7.3, and the proof of Proposition 4.2 in 7.1.

\section{Generic behavior of a Filippov system around a fold-fold singu- larity}

Let $\mathcal{Z}=\mathcal{Z}^{r}, r \geq 1$ be the set of all planar Filippov systems defined in a bounded neighborhood $\mathcal{U} \subset \mathbb{R}^{2}$ of the origin, that is

$$
Z(x, y)=\left\{\begin{array}{ll}
X(x, y), & f(x, y)>0 \\
Y(x, y), & f(x, y)<0
\end{array},\right.
$$

where $X=\left(X^{1}, X^{2}\right), Y=\left(Y^{1}, Y^{2}\right), f \in \mathfrak{X}^{r}(\mathcal{U}), r \geq 1$. As we want to study local singularities we assume $f(x, y)=y$ and that the dynamics on the discontinuity curve $\Sigma=\mathcal{U} \cap f^{-1}(0)$ is given by the Filippov convention. We consider $\mathcal{Z}=\mathfrak{X}^{r} \times \mathfrak{X}^{r}$ with the product $\mathcal{C}^{r}$ topology.

Recall that, by the Filippov convention, as can be seen in [Fil03], the discontinuity curve is decomposed as the closure of the following regions:

$$
\begin{aligned}
& \Sigma^{c}=\left\{(x, 0) \in \Sigma: X^{2} \cdot Y^{2}(x, 0)>0\right\}, \\
& \Sigma^{s}=\left\{(x, 0) \in \Sigma: X^{2}(x, 0)<0 \text { and } Y^{2}(x, 0)>0\right\}, \\
& \Sigma^{e}=\left\{(x, 0) \in \Sigma: X^{2}(x, 0)>0 \text { and } Y^{2}(x, 0)<0\right\} .
\end{aligned}
$$

The flow through a point $p$ in the crossing region is the concatenation of the flow of $X$ and $Y$ through $p$ in a consistent way. Over the regions $\Sigma^{s, e}$, using $x$ as a variable in $\Sigma$, the flow is given by the sliding vector field, denoted by $Z^{s}$ and given by

$$
Z^{s}(x)=\frac{Y^{2} \cdot X^{1}-X^{2} \cdot Y^{1}}{Y^{2}-X^{2}}(x, 0)=\frac{\operatorname{det} Z}{Y^{2}-X^{2}}(x, 0) .
$$

where

$$
\operatorname{det} Z(p)=\left(X^{1} \cdot Y^{2}-X^{2} \cdot Y^{1}\right)(p), p \in \mathbb{R}^{2}
$$

Definition 2.1. The point $p=\left(x_{p}, 0\right) \in \Sigma^{s, e}$ is a pseudo-equilibrium of $Z$ if $Z^{s}\left(x_{p}\right)=0$ and it is a hyperbolic pseudo-equilibrium of $Z^{s}$, if $\left(Z^{s}\right)^{\prime}\left(x_{p}\right) \neq 0$. Moreover,

- $p$ is a pseudo-node if $\left(Z^{s}\right)^{\prime}\left(x_{p}\right)<0$ and $p \in \Sigma^{s}$ or $\left(Z^{s}\right)^{\prime}\left(x_{p}\right)>0$ and $p \in \Sigma^{e}$;

- $p$ is a pseudo-saddle if $\left(Z^{s}\right)^{\prime}\left(x_{p}\right)<0$ and $p \in \Sigma^{e}$ or $\left(Z^{s}\right)^{\prime}\left(x_{p}\right)>0$ and $p \in \Sigma^{s}$.

It follows from (2.2) that $\left(x_{p}, 0\right) \in \Sigma^{e, s}$ is a pseudo-equilibrium if, and only if, $\operatorname{det} Z\left(x_{p}, 0\right)=0$. Moreover, the stability of a pseudo-equilibrium $\left(x_{p}, 0\right) \in \Sigma^{e, s}$ is determined by

$$
\left(Z^{s}\right)^{\prime}\left(x_{p}\right)=\frac{(\operatorname{det} Z)_{x}}{Y^{2}-X^{2}}\left(x_{p}, 0\right) .
$$

When $p \in \Sigma^{c, s, e}$, the vector fields $X$ and $Y$ are transverse to $\Sigma$ at the point $p$, otherwise we have a tangency or fold point. In this paper we are going to deal with fold points.

Notation 2.2. During this paper, given a function $h \in \mathfrak{X}^{r}(\mathcal{U})$, we will denote its partial derivatives by $h_{x}=\frac{\partial h}{\partial x}, h_{y}=\frac{\partial h}{\partial y}, h_{x x}=\frac{\partial^{2} h}{\partial x^{2}}$, etc. 
Definition 2.3. $p \in \Sigma$ is a fold point of $X$ if $X f(p)=X^{2}(p)=0$ and $X(X f)(p)=X_{x}^{2}(p) \cdot X^{1}(p) \neq$ 0 . The fold is visible if $X(X f)(p)>0$ and it is invisible if $X(X f)(p)<0$. Analogously, a fold point $p \in \Sigma$ of $Y$ satisfies $Y f(p)=Y^{2}(p)=0$, and it is visible if $Y(Y f)(p)<0$ and invisible if $Y(Y f)(p)>0$.

Our purpose is to study vector fields $Z \in \mathcal{Z}$ having a fold-fold singularity, which we assume, without loss of generality, that is at the origin $\mathbf{0}=(0,0) \in \Sigma$. That is, using that $f(x, y)=y$ :

$$
\begin{aligned}
& \left\{\begin{array}{l}
X f(\mathbf{0})=X^{2}(\mathbf{0})=0 \\
X(X f)(\mathbf{0})=X_{x}^{2}(\mathbf{0}) \cdot X^{1}(\mathbf{0}) \neq 0
\end{array}\right. \\
& \left\{\begin{array}{l}
Y f(\mathbf{0})=Y^{2}(\mathbf{0})=0 \\
Y(Y f)(\mathbf{0})=Y_{x}^{2}(\mathbf{0}) \cdot Y^{1}(\mathbf{0}) \neq 0
\end{array}\right.
\end{aligned}
$$

The fold-fold singularity has been studied in [KRG03] and [GST11 by considering some normal forms for the Filippov vector fields and their unfoldings. In this section we present a detailed study of the bifurcation diagrams of these singularities, proving that the set of the fold-fold singularities, under some generic conditions, is a codimension one embedded submanifold of $\mathcal{Z}$.

Let $\Xi_{0} \subset \mathcal{Z}$ the set of all locally $\Sigma$-structurally stable Filippov systems defined on $\mathcal{U}$, that is, given $Z \in \Xi_{0} \subset \mathcal{Z}$ there exists a neighborhood $\mathcal{U} \subset \mathcal{Z}$ such that for all $\tilde{Z} \in \mathcal{U}, \tilde{Z}$ is topologically equivalent to $Z$, equivalently, there exists a homeomorphism $h$ which maps trajectories of $Z$ in trajectories of $\tilde{Z}$, preserving the regions of $\Sigma$ and the sliding vector field (see [GST11).

Definition 2.4. Consider $\mathcal{Z}_{1}=\mathcal{Z} \backslash \Xi_{0}$. Let $Z, \tilde{Z} \in \mathcal{Z}_{1}$. We say that two unfoldings $Z_{\delta}$ and $\tilde{Z}_{\tilde{\delta}}$, of $Z$ and $\tilde{Z}$ respectively, are weak equivalent if there exists a homeomorphic change of parameters $\tilde{\delta}=\mu(\delta)$, such that, for each $\delta$ the vector fields $Z_{\delta}$ and $\tilde{Z}_{\mu(\delta)}$ are locally $\Sigma$-equivalent. Moreover, given an unfolding $Z_{\delta}$ of $Z$ it is said to be a versal unfolding if every other unfolding $Z_{\tilde{\delta}}$ of $Z$ is weak equivalent to $Z_{\delta}$.

Definition 2.5. We define $\Lambda^{F} \subset \mathcal{Z}_{1}$ as the set of Filippov systems which have a locally $\Sigma$-structurally stable fold-fold. More precisely, given $Z \in \Lambda^{F}$ there exists a neighborhood $\mathcal{V}_{Z}$ such that given $\tilde{Z} \in \mathcal{V}_{Z} \cap \mathcal{Z}_{1}$ then $Z$ is locally $\Sigma$-equivalent to $\tilde{Z}$ and their versal unfoldings are weak equivalent.

This section is devoted to prove the following theorem:

Theorem 2.6. Consider $\Lambda^{F} \subset \mathcal{Z}_{1}$ the set of all Filippov systems $Z$ which have a $\Sigma$-structurally stable fold-fold singularity in the induced topology on $\mathcal{Z}_{1}$. Then $Z \in \mathcal{Z}_{1}$ belongs to $\Lambda^{F}$ if and only if satisfies one of the following conditions:

(A) it is a visible-visible fold;

(B) it is an invisible-invisible fold which is a non degenerated fixed point for the generalized Poincaré return map. See (2.15) for a precise definition;

(C) it is a visible-invisible fold and, in the case where the sliding vector field $Z^{s}(x)$ is defined, it must satisfy

$$
\gamma:=Z^{s}(0) \neq 0
$$

In addition, $\Lambda^{F}$ is a codimension one embedded submanifold of $\mathcal{Z}$.

Remark 2.7. Theorem 2.6 says that given a vector filed $Z$ satisfying the conditions of the Theorem if we consider unfoldings of the form:

$$
Z^{\alpha}=Z+\alpha \tilde{Z}+\mathcal{O}\left(\alpha^{2}\right)
$$

they all are equivalent if they are versal. The condition for this unfoldings to be versal, roughly speaking, is that for $\alpha \neq 0, Z^{\alpha}$ has not a fold-fold singularity. As we will see during the proof of this theorem this is equivalent to satisfy:

$$
\frac{\tilde{Y}^{2}(\mathbf{0})}{Y_{x}^{2}(\mathbf{0})}-\frac{\tilde{X}^{2}(\mathbf{0})}{X_{x}^{2}(\mathbf{0})} \neq 0
$$


The rest of this section is devoted to prove this theorem. As we are going to deal with local singularities, we will always work in a neighborhood of the origin without explicit mention.

Next lemma, whose proof is straightforward, characterizes the cases where there is a region of sliding around the fold-fold point.

Lemma 2.8. Suppose that the origin is a fold-fold point for $Z \in \mathcal{Z}$, then:

- If the folds have the same visibility, $\Sigma=\overline{\Sigma^{c}}$ if $X^{1} \cdot Y^{1}(\mathbf{0})<0$ and $\Sigma=\overline{\Sigma^{e} \cup \Sigma^{s}}$ if $X^{1} \cdot Y^{1}(\mathbf{0})>$ 0 .

- If the folds have opposite visibility, $\Sigma=\overline{\Sigma^{c}}$ if $X^{1} \cdot Y^{1}(\mathbf{0})>0$ and $\Sigma=\overline{\Sigma^{e} \cup \Sigma^{s}}$ if $X^{1} \cdot Y^{1}(\mathbf{0})<0$.

In the case that the sliding vector field $(2.2)$ is defined around the fold-fold $(0,0)$, by Definition 2.3 and Lemma 2.8, we have $\left(Y_{x}^{2}-X_{x}^{2}\right)(\mathbf{0}) \neq 0$. Therefore in this case, even if $Z^{s}$ is not defined at $x=0$, one can extend it by the L'Hôpital's rule:

$$
\gamma:=Z^{s}(0)=\lim _{x \rightarrow 0} Z^{s}(x)=\frac{(\operatorname{det} Z)_{x}}{\left(Y_{x}^{2}-X_{x}^{2}\right)}(\mathbf{0}) .
$$

Thus the sliding vector field $Z^{s}$ is well defined at the origin.

Lemma 2.9. Suppose that the origin is a fold-fold point of $Z=(X, Y)$ then

(a) if both folds are visible, we have $(\operatorname{det} Z)_{x}(\mathbf{0})>0$ when $X^{1} \cdot Y^{1}(\mathbf{0})<0$ and $(\operatorname{det} Z)_{x}(\mathbf{0})<0$ when $X^{1} \cdot Y^{1}(\mathbf{0})>0$;

(b) if both folds are invisible, we have $(\operatorname{det} Z)_{x}(\mathbf{0})<0$ when $X^{1} \cdot Y^{1}(\mathbf{0})<0$ and $(\operatorname{det} Z)_{x}(\mathbf{0})>0$ when $X^{1} \cdot Y^{1}(\mathbf{0})>0$;

(c) if the folds have opposite visibility and $X^{1} \cdot Y^{1}(\mathbf{0})<0$ and $Z^{s}$ satisfies hypothesis (2.7), then $(\operatorname{det} Z)_{x}(\mathbf{0}) \neq 0$. However, one can not decide, a priori, its sign.

Proof. It follows from (2.5) (2.6) and the fact that $(\operatorname{det} Z)_{x}(\mathbf{0})=\left(X^{1} \cdot Y_{x}^{2}-Y^{1} \cdot X_{x}^{2}\right)(\mathbf{0})$.

Corolary 2.10. If the sliding vector field $Z^{s}$ is defined around the fold-fold point, we have:

- If the folds have the same visibility, then $\operatorname{sgn}(\gamma)=\operatorname{sgn}\left(X^{1}(\mathbf{0})\right)$;

- If the folds have opposite visibility, then $\operatorname{sgn}(\gamma)=-\operatorname{sgn}\left(X^{1}(\mathbf{0}) \cdot(\operatorname{det} Z)_{x}(\mathbf{0})\right)$,

where $\gamma$ is given in (2.9).

Corolary 2.11. Let $Z_{0}=\left(X_{0}, Y_{0}\right) \in \mathcal{Z}$ having a fold-fold at the origin satisfying the same hypotheses of Lemma 2.9. Then there exist neighborhoods $Z_{0} \in \mathcal{U}_{0} \subset \mathcal{Z}$ and $\mathbf{0} \in \mathcal{I}_{0} \subset \Sigma$ such that for each $Z \in \mathcal{U}_{0}$ there exists a unique $P(Z) \in \mathcal{I}_{0}$ such that $\operatorname{det} Z(P(Z), 0)=0$ and $\operatorname{sgn}\left((\operatorname{det} Z)_{x}(P(Z), 0)\right)=\operatorname{sgn}\left(\left(\operatorname{det} Z_{0}\right)_{x}(\mathbf{0})\right)$.

Proof. Let $Z_{0} \in \mathcal{Z}$ satisfying the hypothesis of Lemma 2.9. Let $\xi$ be the Frechet differentiable map

$$
\begin{array}{rlc}
\xi: \mathcal{Z} \times \mathbb{R} & \rightarrow & \mathbb{R} \\
(Z, x) & \mapsto & \operatorname{det} Z(x, 0)
\end{array}
$$

As $Z_{0}$ has a fold-fold at the origin, then $\xi\left(Z_{0}, 0\right)=\operatorname{det} Z_{0}(\mathbf{0})=0$ and by the Lemma 2.9 we have $\xi_{x}\left(Z_{0}, 0\right)=\left(\operatorname{det} Z_{0}\right)_{x}(\mathbf{0}) \neq 0$. By the Implicit Function Theorem we obtain neighborhoods $Z_{0} \in \mathcal{U}_{0} \subset \mathcal{Z}$ and $0 \in \mathcal{I}_{0} \subset \Sigma$ and a Frechet differentiable map $P: \mathcal{U}_{0} \rightarrow \mathcal{I}_{0}$ satisfying $\xi(Z, x)=0$ if, and only if, $x=P(Z)$. That is $\xi(Z, P(Z))=\operatorname{det} Z(P(Z), 0)=0$ for all $Z \in \mathcal{U}_{0}$. Moreover, we can assume without loss of generality that in this neighborhood we have $\operatorname{sgn}\left((\operatorname{det} Z)_{x}(P(Z), 0)\right)=$ $\operatorname{sgn}\left(\left(\operatorname{det} Z_{0}\right)_{x}(\mathbf{0})\right)$. 
In the case of the invisible fold-fold it is natural to consider the first return map ([Tei81] $)$. Next proposition, whose proof is straightforward and can be found in [Lar15], gives the main term of the Taylor expansion of the Poincaré map near a tangency point.

Proposition 2.12 (PoincarÃl map for $X$ at a point $\left.\left(x_{0}, y_{0}\right) \in \Sigma_{y_{0}}\right)$. Let $X$ be a smooth vector field having a fold point at $p_{0}=\left(x_{0}, y_{0}\right) \in \Sigma_{y_{0}}=\left\{\left(x, y_{0}\right): x \in \mathcal{I}\right\}$, where $\mathcal{I}=\mathcal{I}\left(y_{0}\right)$ is a neighborhood of $x_{0}$.

Then the PoincarÃl map $\phi_{X}^{p_{0}}$ is given by

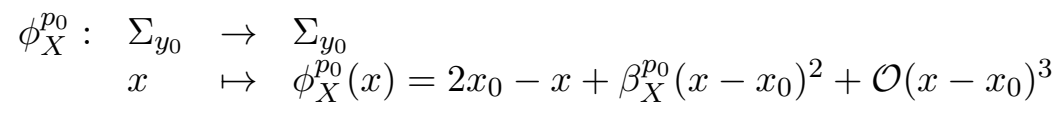

where

$$
\beta_{X}^{p_{0}}=\frac{1}{3}\left[-\frac{X_{x x}^{2}}{X_{x}^{2}}+2 \frac{X_{x}^{1}}{X^{1}}+2 \frac{X_{y}^{2}}{X^{1}}\right]\left(p_{0}\right) .
$$

Suppose that the vector field $Z$ has an invisible fold-fold point at $\mathbf{0} \in \Sigma$ with $\Sigma=\overline{\Sigma^{c}}$. Then, it has sense to consider the first return map that, for convenience, we define on $\Sigma^{-}=\{(x, 0), x \in \mathcal{I}$ : $x<0\}$

$$
\phi_{Z}: \Sigma^{-} \rightarrow \Sigma^{-},
$$

by setting $p_{0}=\mathbf{0}$ and composing appropriately the PoincarÃl maps for $X$ and $Y$. Using Proposition 2.12 we obtain

$$
\begin{aligned}
& \phi_{Z}(x)=\phi_{Y} \circ \phi_{X}(x)=x+\left(\beta_{Y}-\beta_{X}\right) x^{2}+\mathcal{O}\left(x^{3}\right), \text { if } X_{1}(0)>0 \\
& \phi_{Z}(x)=\phi_{X} \circ \phi_{Y}(x)=x+\left(\beta_{X}-\beta_{Y}\right) x^{2}+\mathcal{O}\left(x^{3}\right), \text { if } X_{1}(0)<0
\end{aligned}
$$

The generic condition stated in item $(\mathrm{B})$ of Theorem 2.6 for the invisible fold-fold is that

$$
\mu_{Z}=\beta_{Y}-\beta_{X} \neq 0 .
$$

Remark 2.13. If $X^{1}(\mathbf{0})>0, \phi_{Z}$ is defined in $\Sigma^{-}$and is given by (2.13). The origin is an attractor fixed point for $\phi_{Z}$ if $\mu_{Z}=\beta_{Y}-\beta_{X}>0$ and it is a repellor in case $\mu_{Z}=\beta_{Y}-\beta_{X}<0$. Analogously for the case $X^{1}(\mathbf{0})<0$. Nevertheless, it is important to stress that, as $\phi_{Z}^{\prime}(0)=1$, the origin is never a hyperbolic fixed point of the first return map $\phi_{Z}$ even in the generic case. This will have consequences latter in section 3 when we study the regularization of the vector field $Z$.

Remark 2.14. An important detail that had not been observed in [GST11] and [KRG03] is that, even in the case $\Sigma=\overline{\Sigma^{s} \cup \Sigma^{e}}$, one needs to consider the first return map and impose the same generic condition (2.15). Even though the first return map has no dynamical meaning in this case, the pseudo-cycles, which correspond to fixed points of $\phi_{Z}$, must be preserved by $\Sigma$-equivalences. This map will be used in Section 2.2 when we study the unfolding of an invisible fold-fold satisfying $X^{1} \cdot Y^{1}(\mathbf{0})>0$ and in this case we consider $\phi_{Z}=\phi_{Y} \circ \phi_{X}$ independently of the sign of $X^{1}(\mathbf{0})$.

Now, we are able to state and prove that conditions $(\mathrm{A})$ to $(\mathrm{C})$ in Theorem 2.6 which characterize a codimension one embedded submanifold in $\mathcal{Z}$.

Proposition 2.15. The set $\tilde{\Lambda}^{F} \subset \mathcal{Z}$ of all Filippov systems which have a fold-fold at the origin satisfying the hypothesis (A), (B) or (C) in Theorem 2.6 is an embedded co-dimension one submanifold of $\mathcal{Z}$. That is, for each $Z^{0} \in \tilde{\Lambda}^{F}$ there exist a map $\lambda: \mathcal{V}_{0} \rightarrow \mathbb{R}$ where $\mathcal{V}_{0} \subset \mathcal{Z}$ is a neighborhood of $Z^{0}$ and $Z^{0} \in \lambda^{-1}(0)=\mathcal{V}_{0} \cap \tilde{\Lambda}^{F}$ and $D \lambda_{Z^{0}} \neq 0$.

Proof. Consider $Z^{0} \in \tilde{\Lambda}^{F}$. Let $\mathcal{U}_{0} \subset \mathcal{Z}$ be a neighborhood of $Z^{0}$ sufficiently small such that in this neighborhood the sign of $X^{1}(x, 0), Y^{1}(x, 0), X_{x}^{2}(x, 0)$ and $Y_{x}^{2}(x, 0)$ is constant for $x \in \mathcal{I}_{0} \subset$ $\mathbb{R}$. Moreover, if $Z^{0}$ satisfies the hypothesis of Lemma 2.9. suppose that $\operatorname{sgn}\left((\operatorname{det} Z)_{x}(x, 0)\right)=$ $\left.\operatorname{sgn}\left(\left(\operatorname{det} Z^{0}\right)_{x}(\mathbf{0})\right)\right)$ for all $Z \in \mathcal{U}_{0}$ and $x \in \mathcal{I}_{0}$. 
Consider the following Frechet differentiable map

$$
\begin{aligned}
\xi: \mathcal{U}_{0} \times \mathbb{R}^{2} & \rightarrow \mathbb{R}^{2} \\
(Z,(p, q)) & \mapsto\left(X^{2}(p, 0), Y^{2}(q, 0)\right) .
\end{aligned}
$$

Since $\mathbf{0} \in \Sigma$ is a fold-fold point $\xi\left(Z^{0}, \mathbf{0}\right)=\mathbf{0}$ and by $(2.5)$ and $(2.6)$

$$
\operatorname{det} D_{(p, q)} \xi\left(Z^{0}, \mathbf{0}\right)=\left(X^{0}\right)_{x}^{2} \cdot\left(Y^{0}\right)_{x}^{2}(\mathbf{0}) \neq 0 .
$$

Using the Implicit Function Theorem for $\xi$ there exist $\mathcal{V}_{0}^{*} \subset \mathcal{U}_{0}$ and a map

$$
T: Z=(X, Y) \in \mathcal{V}_{0}^{*} \subset \mathcal{U}_{0} \mapsto\left(T_{X}, T_{Y}\right) \in \mathcal{I}_{0} \times \mathcal{I}_{0} \subset \mathbb{R}^{2}
$$

defined in a path connected open set such that $\xi(Z,(p, q))=(0,0)$ if, and only if, $(p, q)=\left(T_{X}, T_{Y}\right)$. In other words, $\xi(Z, T(Z))=\mathbf{0}$ for every $Z \in \mathcal{V}_{0}^{*}$. That is, $X$ and $Y$ have a fold point $\left(T_{X}, 0\right)$ and $\left(T_{Y}, 0\right)$ near the origin with the same visibility as the origin has for $X^{0}$ and $Y^{0}$.

To show that $\tilde{\Lambda}^{F}$ is a submanifold let consider the Frechet differentiable map

$$
\begin{aligned}
\lambda_{*}: \mathcal{V}_{0}^{*} & \rightarrow \mathbb{R} \\
Z & \mapsto T_{X}-T_{Y} .
\end{aligned}
$$

It is clear that $Z \in \mathcal{V}_{0}^{*}$ has a fold-fold point near the origin if, and only if, $Z \in \lambda_{*}^{-1}(0)$. Moreover, it is easy to see that when $Z^{0}$ satisfies items (A) or (C) then every $Z \in \lambda_{*}^{-1}(0)$ also belongs to $\tilde{\Lambda}^{F}$ and the fold-fold type is preserved. In this case, set $\mathcal{V}_{0}=\mathcal{V}_{0}^{*}$.

When $Z^{0}$ satisfies (B) with $\mu_{Z^{0}} \neq 0$ (2.15) then there exists a neighborhood $\tilde{\mathcal{V}}_{0}^{1}=\mathcal{V}_{0}^{1} \cap \mathcal{Z}_{1}$, with $\mathcal{V}_{0}^{1} \subset \mathcal{Z}$, such that $\operatorname{sgn}\left(\mu_{Z}\right)=\operatorname{sgn}\left(\mu_{Z^{0}}\right)$, for all $Z \in \mathcal{V}_{0}^{1}$. Therefore the fold-fold point has the same attractivity to $\phi_{Z}$ as the origin has to $\phi_{Z^{0}}$. In this case set $\mathcal{V}_{0}=\mathcal{V}_{0}^{*} \cap \mathcal{V}_{0}^{1}$. that

Consider then the map $\lambda=\left.\lambda_{*}\right|_{\mathcal{V}_{0}}$. It follows that $\lambda^{-1}(\mathbf{0})=\mathcal{V}_{0} \cap \tilde{\Lambda}^{F}$. To finish our proof, observe

$$
D \lambda_{Z^{0}}(Z)=D\left(T_{X}-T_{Y}\right)_{Z^{0}}(Z)=\frac{X^{2}(\mathbf{0})}{\left(X^{0}\right)_{x}^{2}(\mathbf{0})}-\frac{Y^{2}(\mathbf{0})}{\left(Y^{0}\right)_{x}^{2}(\mathbf{0})},
$$

and therefore the resulting map is a non-vanishing linear map, what proves the desired result.

The next step to finish the proof of Theorem 2.6 is to prove that any versal unfoldings of $Z^{0} \in \lambda^{-1}(0)$ are weak equivalent and consequently $\Lambda^{F}=\Lambda^{F}$. As we will see the behavior of the unfolding depends on the sign of $X^{1} \cdot Y^{1}(\mathbf{0})$, but the study is completely analogous for $X^{1}(\mathbf{0})$ positive or negative. Therefore, in what follows, we assume that $X^{1}(\mathbf{0})>0$.

Consider $\gamma: \alpha \in\left(-\alpha_{0}, \alpha_{0}\right) \mapsto Z^{\alpha} \in \mathcal{V}_{0}$ with $\alpha_{0} \ll 1$, a versal unfolding of $Z^{0}$, where $\mathcal{V}_{0}$ is the neighborhood given in Proposition 2.15. Since $\gamma$ is transverse to $\lambda^{-1}(0)$ at $Z^{0}$, and the derivative of $\lambda$ is given in (2.17), one can write $Z^{\alpha}=Z^{0}+\tilde{Z} \alpha+\mathcal{O}(\alpha)^{2}$ with

$$
\frac{\tilde{Y}^{2}(\mathbf{0})}{\left(Y^{0}\right)_{x}^{2}(\mathbf{0})}-\frac{\tilde{X}^{2}(\mathbf{0})}{\left(X^{0}\right)_{x}^{2}(\mathbf{0})} \neq 0 .
$$

This condition ensures that for $\alpha \neq 0$ small, the vector field $Z^{\alpha}$ has not a fold-fold.

Moreover, since $\lambda^{-1}(0) \subset \mathcal{V}_{0}$ is a codimension one embedded submanifold and $\mathcal{V}_{0}$ is path connected the set $\lambda^{-1}(0)$ splits $\mathcal{V}_{0}$ in two connected components, namely, $\mathcal{V}_{0}^{ \pm}=\lambda^{-1}\left(\mathbb{R}^{ \pm}\right)$. Therefore in the sequel we suppose that $\gamma\left(-\alpha_{0}, 0\right) \subset \mathcal{V}_{0}^{-}$and $\gamma\left(0, \alpha_{0}\right) \subset \mathcal{V}_{0}^{+}$.

By Proposition 2.15, applied to the particular curve $\gamma(\alpha)$, for each $\alpha \in\left(-\alpha_{0}, \alpha_{0}\right)$ there exist $T_{X}^{\alpha}, T_{Y}^{\alpha} \in \Sigma$ near the origin given by

$$
T_{X}^{\alpha}=-\frac{\tilde{X}^{2}(\mathbf{0})}{\left(X^{0}\right)_{x}^{2}(\mathbf{0})} \alpha+\mathcal{O}\left(\alpha^{2}\right) \text { and } T_{Y}^{\alpha}=-\frac{\tilde{Y}^{2}(\mathbf{0})}{\left(Y^{0}\right)_{x}^{2}(\mathbf{0})} \alpha+\mathcal{O}\left(\alpha^{2}\right) .
$$

Then to assume $T_{X}^{\alpha}-T_{Y}^{\alpha}>0$ for $\alpha>0$ is equivalent to

$$
\frac{\tilde{Y}^{2}(\mathbf{0})}{\left(Y^{0}\right)_{x}^{2}(\mathbf{0})}-\frac{\tilde{X}^{2}(\mathbf{0})}{\left(X^{0}\right)_{x}^{2}(\mathbf{0})}>0 .
$$


Note that the points $\left(T_{X}^{\alpha}, 0\right)$ and $\left(T_{Y}^{\alpha}, 0\right)$ are the tangency points of the vector field $Z^{\alpha}$. Therefore, assumption (2.19) ensures that the tangency of the vector field $X^{\alpha}$ is on the left of the tangency of $Y^{\alpha}$ when $\alpha<0$ and otherwise when $\alpha>0$.

Once we show that any unfolding has the same phase portrait, a systematic construction of the homeomorphism giving the topological equivalences between them can be easily done using the ideas of [GST11]. Then any two unfoldings are weak equivalent. In conclusion, joining the result of Proposition 2.15 and the following propositions 2.16, 2.17, 2.18, 2.19 and 2.20, we prove Theorem 2.6. In what follows, in order to avoid a huge amount of cases, we fix $X^{1}(\mathbf{0})>0$.

\subsection{The versal unfolding of a visible fold-fold singularity}

Proposition 2.16. Let $Z \in \Lambda^{F}$ satisfying condition $(A)$ of Theorem 2.6 and $X^{1} \cdot Y^{1}(\mathbf{0})>0$. Let $\mathcal{V}_{0}$ be the neighborhood given by Proposition 2.15. Then any smooth curve

$$
\gamma: \alpha \in\left(-\alpha_{0}, \alpha_{0}\right) \mapsto Z^{\alpha} \in \mathcal{V}_{0}
$$

which is transverse to $\Lambda^{F}$ at $\gamma(0)=Z$ leads to the same topological behaviors in $\mathcal{V}_{0}^{+}$and in $\mathcal{V}_{0}^{-}$. Any vector field $Z^{\alpha}$ has two visible fold points with a crossing region between them. In the sliding and escaping regions there are no pseudo-equilibrium Therefore, there exists a weak equivalence between any two unfoldings of $Z$.

Proof. Since $X^{1} \cdot Y^{1}(\mathbf{0})>0, \Sigma=\overline{\Sigma^{s} \cup \Sigma^{e}}$ for $\alpha=0$. For $\alpha \neq 0$, we know the existence of the folds $T_{X}^{\alpha}$ and $T_{Y}^{\alpha}$ given in (2.18) Observe that for $\alpha \neq 0$ we have $X^{\alpha 2}(x, 0)<0$ if $x<T_{X}^{\alpha}$ and $Y^{\alpha 2}(x, 0)<0$ if $x>T_{Y}^{\alpha}$. Analogously, $X^{\alpha 2}(x, 0)>0$ if $x>T_{X}^{\alpha}$ and $Y^{\alpha 2}(x, 0)>0$ if $x<T_{Y}^{\alpha}$, see Figure 1. Therefore, a crossing region appears between the folds and the discontinuity curve is decomposed as $\Sigma=\Sigma^{s} \cup \Sigma^{c} \cup \Sigma^{e}$, as follows:

$$
\begin{aligned}
& \Sigma^{s}=\left\{x \in \Sigma: x<\min \left\{T_{X}^{\alpha}, T_{Y}^{\alpha}\right\}\right\}, \\
& \Sigma^{c}=\left\{x \in \Sigma: x \in\left(\min \left\{T_{X}^{\alpha}, T_{Y}^{\alpha}\right\}, \max \left\{T_{X}^{\alpha}, T_{Y}^{\alpha}\right\}\right)\right\}, \\
& \Sigma^{e}=\left\{x \in \Sigma: x>\max \left\{T_{X}^{\alpha}, T_{Y}^{\alpha}\right\}\right\} .
\end{aligned}
$$

Observe that

$$
\operatorname{det} Z^{\alpha}\left(T_{X}^{\alpha}, 0\right)=X^{\alpha 1} \cdot Y^{\alpha 2}\left(T_{X}^{\alpha}, 0\right) \text { and } \operatorname{det} Z^{\alpha}\left(T_{Y}^{\alpha}, 0\right)=-X^{\alpha 2} \cdot Y^{\alpha 1}\left(T_{Y}^{\alpha}, 0\right) .
$$

Moreover, by definition (2.2) of the sliding vector field and using the fact of $T_{X, Y}^{\alpha}$ are fold points and $\operatorname{sgn}\left(X^{\alpha 1}(x, 0)\right)=\operatorname{sgn}\left(Y^{\alpha 1}(x, 0)\right)>0$, we have

$$
\operatorname{sgn}\left(\left(Z^{\alpha}\right)^{s}\left(T_{X}^{\alpha}\right)\right)=\operatorname{sgn}\left(\left(Z^{\alpha}\right)^{s}\left(T_{Y}^{\alpha}\right)\right)=\operatorname{sgn}\left(X^{\alpha 1}(\mathbf{0})\right)>0 .
$$

Then the sliding vector field of $Z^{\alpha}$ near $Z^{0}$ satisfies $\operatorname{sgn}\left(\left(Z^{\alpha}\right)^{s}(x)\right)=\operatorname{sgn}\left(X^{1}(\mathbf{0})\right)$. In particular there are no pseudo-equilibrium

By (2.20), for $\alpha<0$, the sliding vector field is defined for $x<T_{X}^{\alpha}$ and for $x>T_{Y}^{\alpha}$. In addition, between the folds we have that $X^{\alpha 2}, Y^{\alpha 2}>0$. For $\alpha>0$, the sliding vector field is defined for $x<T_{Y}^{\alpha}$ and $x>T_{X}^{\alpha}$ and between the folds $X^{\alpha 2}, Y^{\alpha 2}<0$.

This proves that any unfolding of $Z \in \Lambda^{F}$ satisfying $(A)$ leads to vector fields with exactly the same behavior, that is, the same $\Sigma$-regions and singularities. A sketch of a versal unfolding of the visible fold-fold satisfying $X^{1} \cdot Y^{1}(\mathbf{0})>0$ can be seen in Figure 1 .

Proposition 2.17. Let $Z \in \Lambda^{F}$ satisfying condition $(A)$ of Theorem 2.6 and $X^{1} \cdot Y^{1}(\mathbf{0})<0$. Let $\mathcal{V}_{0}$ be the neighborhood given by Proposition 2.15. Then any smooth curve

$$
\gamma: \alpha \in\left(-\alpha_{0}, \alpha_{0}\right) \mapsto Z^{\alpha} \in \mathcal{V}_{0}
$$

which is transverse to $\Lambda^{F}$ at $\gamma(0)=Z$ leads to the same behaviors in $\mathcal{V}_{0}^{+}$and in $\mathcal{V}_{0}^{-}$. If $Z^{\alpha} \in \mathcal{V}_{0}^{-}$ $\left(Z^{\alpha} \in \mathcal{V}_{0}^{+}\right)$, it has two visible fold points with a escaping (sliding) region between them, whose sliding vector field has a pseudo-saddle $Q(\alpha)=(x(\alpha), 0)$. Therefore, there exists a weak equivalence between any two unfoldings of $Z$. 

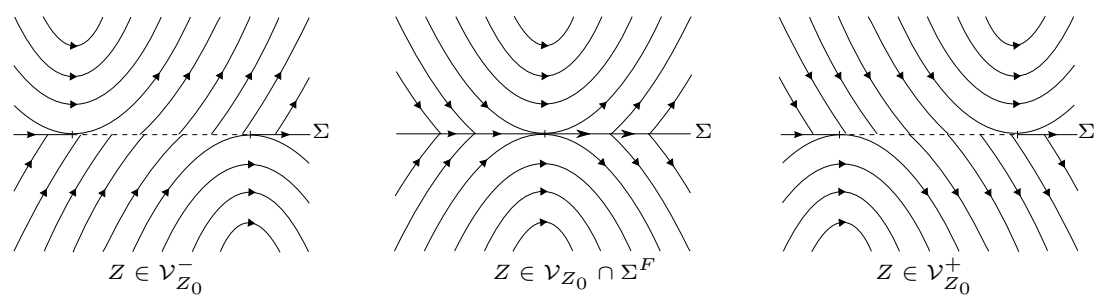

Figure 1: Versal unfolding for a visible fold-fold: $X^{1} \cdot Y^{1}(\mathbf{0})>0$

Proof. Since $X^{1} \cdot Y^{1}(\mathbf{0})<0, \Sigma=\overline{\Sigma^{c}}$ for $\alpha=0$. For $\alpha \neq 0$, we know the existence of the folds $T_{X}^{\alpha}$ and $T_{Y}^{\alpha}$ given in (2.18), Observe that $X^{\alpha 2}(x, 0)<0$ if $x<T_{X}^{\alpha}$ and $Y^{\alpha 2}(x, 0)>0$ if $x>T_{Y}^{\alpha}$. Analogously, $X^{\alpha 2}(x, 0)>0$ if $x>T_{X}^{\alpha}$ and $Y^{\alpha 2}(x, 0)<0$ if $x<T_{Y}^{\alpha}$, see Figure 2 . Therefore, a piece of sliding $(\alpha>0)$ or escaping $(\alpha<0)$ region appear between the folds.

$$
\begin{aligned}
\Sigma^{c} & =\left\{x \in \Sigma: x<\min \left\{T_{X}^{\alpha}, T_{Y}^{\alpha}\right\}\right\} \cup\left\{x \in \Sigma: x>\max \left\{T_{X}^{\alpha}, T_{Y}^{\alpha}\right\}\right\} \\
\Sigma \backslash \Sigma^{c}, & = \begin{cases}\Sigma^{e}= & \left.\left\{x \in \Sigma: x \in\left(T_{X}^{\alpha}, T_{Y}^{\alpha}\right)\right\}\right\}, \alpha<0, \\
\Sigma^{s}= & \left.\left\{x \in \Sigma: x \in\left(T_{Y}^{\alpha}, T_{X}^{\alpha}\right)\right\}\right\}, \alpha>0 .\end{cases}
\end{aligned}
$$

Observe that $\operatorname{det} Z^{\alpha}\left(T_{X}^{\alpha}, 0\right) \cdot \operatorname{det} Z^{\alpha}\left(T_{Y}^{\alpha}, 0\right)<0$ for $\alpha \neq 0$. Then there exists a point $x(\alpha) \in \Sigma^{e, s}$ such $\operatorname{det} Z^{\alpha}(x(\alpha), 0)=0$. Moreover, by Lemma 2.9 we know that $\left(\operatorname{det} Z^{0}\right)_{x}(\mathbf{0})>0$ and therefore by Corolary 2.11, for $\alpha$ small enough $x(\alpha)$ is unique. Therefore $Q(\alpha)=(x(\alpha), 0)$ is a pseudoequilibrium of $\left(Z^{\alpha}\right)^{s}$. Moreover, by $(2.4)$

$$
\left(\left(Z^{\alpha}\right)^{s}\right)^{\prime}(x(\alpha))=\frac{\left(\operatorname{det} Z^{\alpha}\right)_{x}(Q(\alpha))}{\left(Y^{\alpha 2}-X^{\alpha 2}\right)(Q(\alpha))} .
$$

As $\left(\operatorname{det} Z^{\alpha}\right)_{x}(Q(\alpha))>0$ for $|\alpha| \ll 1,\left(\left(Z^{\alpha}\right)^{s}\right)^{\prime}(x(\alpha))$ is positive if $\alpha>0$ and it is negative if $\alpha<0$. This fact implies that the point $Q(\alpha)$ is a pseudo-saddle of the sliding vector field.

This proves that any unfolding of $Z \in \Lambda^{F}$ satisfying $(A)$ with $X^{1} \cdot Y^{1}(\mathbf{0})<0$ leads to vector fields with exactly the same topological invariants, see Figure 2 .
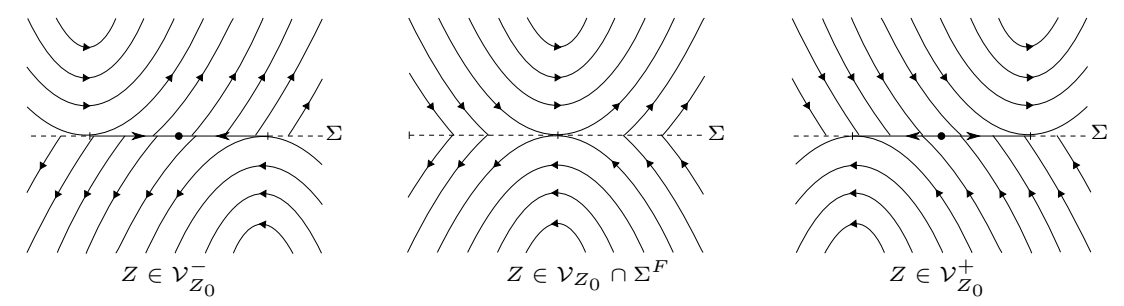

Figure 2: Versal unfolding for a visible fold-fold: $X^{1} \cdot Y^{1}(\mathbf{0})<0$

\subsection{The versal unfolding of a invisible fold-fold singularity}

To study the unfoldings of a Filippov vector field $Z$ having an invisible fold-fold, we need to consider the generalized first return map (2.12) around the fold-fold point. We will have four different types of bifurcations depending on the sign of $X^{1} \cdot Y^{1}(\mathbf{0})$ and the attracting or repelling character of the return map. The case where $\Sigma=\overline{\Sigma^{c}}$ is the so called pseudo-Hopf bifurcation and it was studied in [KRG03 and [GST11] and it is a generic codimension one bifurcation if $\mu_{Z} \neq 0$ (see (2.15).

Proposition 2.18. Let $Z \in \Lambda^{F}$ satisfying condition $(B)$ of Theorem 2.6. $X^{1} \cdot Y^{1}(\mathbf{0})<0$ and $\mu_{Z} \neq 0$ (see(2.15)). Let $\mathcal{V}_{0}$ be the neighborhood given in Proposition 2.15. Then any smooth curve

$$
\gamma: \alpha \in\left(-\alpha_{0}, \alpha_{0}\right) \mapsto Z^{\alpha} \in \mathcal{V}_{0}
$$

which is transverse to $\Lambda^{F}$ at $\gamma(0)=Z$ leads to the same topological behavior in $\mathcal{V}_{0}^{+}$and in $\mathcal{V}_{0}^{-}$ This behavior depends of the sign of $\mu_{Z}$ : 
1. If $\mu_{Z}>0$ :

- Every $Z \in \mathcal{V}_{0}^{-}$has two invisible fold points and there exists a region of sliding between them. The sliding vector field has a stable pseudo-node $Q(\alpha)=(x(\alpha), 0) \in \Sigma^{s}$ which is a global attractor.

- Every $Z \in \mathcal{V}_{0}^{+}$has two invisible fold points and there exists a region of escaping between them. The sliding vector field has a unstable pseudo-node $Q(\alpha)=(x(\alpha), 0) \in \Sigma^{e}$ and there exists a crossing stable periodic orbit $\Gamma^{\alpha}$ which is a global attractor.

2. If $\mu_{Z}<0$ :

- Every $Z \in \mathcal{V}_{0}^{-}$has two invisible fold points and there exists a region of sliding between them. The sliding vector field has a stable pseudo-node $Q(\alpha)=(x(\alpha), 0) \in \Sigma^{s}$ and there exists a crossing unstable periodic $\Gamma^{\alpha}$ orbit which is a global repellor.

- Every $Z \in \mathcal{V}_{0}^{+}$has two invisible fold points and there exists a region of escaping between them. The sliding vector field has a unstable pseudo-node $Q(\alpha)=(x(\alpha), 0) \in \Sigma^{e}$ which is a global repellor.

Therefore, there exists a weak equivalence between any two unfoldings of $Z$.

Proof. Since $X^{1} \cdot Y^{1}(\mathbf{0})<0, \Sigma=\overline{\Sigma^{c}}$ for $\alpha=0$. For $\alpha \neq 0$, for all the points $(x, 0)$ between the folds $T_{X}^{\alpha}$ and $T_{Y}^{\alpha}$ the vector field $Z^{\alpha}$ satisfies $X^{\alpha 2} \cdot Y^{\alpha 2}(x, 0)<0$. Therefore, a piece of sliding (for $\alpha<0$ ) or escaping (for $\alpha>0$ ) region appears between the folds. The discontinuity curve becomes $\Sigma=\overline{\Sigma^{e, s} \cup \Sigma^{c}}$, where

$$
\begin{aligned}
\Sigma^{c} & =\left\{x \in \Sigma: x<\min \left\{T_{X}^{\alpha}, T_{Y}^{\alpha}\right\}\right\} \cup\left\{x \in \Sigma: x>\max \left\{T_{X}^{\alpha}, T_{Y}^{\alpha}\right\}\right\} \\
\Sigma \backslash \Sigma^{c}, & = \begin{cases}\Sigma^{s}= & \left.\left\{x \in \Sigma: x \in\left(T_{X}^{\alpha}, T_{Y}^{\alpha}\right)\right\}\right\}, \alpha<0, \\
\Sigma^{e}= & \left.\left\{x \in \Sigma: x \in\left(T_{Y}^{\alpha}, T_{X}^{\alpha}\right)\right\}\right\}, \alpha>0 .\end{cases}
\end{aligned}
$$

Since we have sliding motion defined on one side of the tangencies and crossing on the other, following [GST11], the fold points are singular tangency points. Using the same argument as in Proposition 2.17, there exists a unique $Q(\alpha) \in \Sigma^{e, s}$ such that $\operatorname{det} Z^{\alpha}(Q(\alpha))=0$. By Lemma 2.9. we have $(\operatorname{det} Z)_{x}(\mathbf{0})<0$. Using the formulas for $\left(Z^{s}\right)^{\prime}(x(\alpha))$ given in $(2.4)$ the pseudo-equilibrium $Q(\alpha)$ is an stable pseudo-node when $\alpha<0$ and it is a unstable pseudo-node when $\alpha>0$.

To give a complete description of the dynamics one needs to analyze the first return map around the fold-fold singularity:

$$
\begin{aligned}
\phi^{\alpha}: \mathcal{D}^{\alpha} & \rightarrow \mathcal{I}^{\alpha} \\
x & \mapsto \phi^{\alpha}(x)=\phi_{Y}^{\alpha} \circ \phi_{X}^{\alpha}(x)
\end{aligned}
$$

where

$$
\begin{aligned}
& \mathcal{D}^{\alpha}=\left\{x \in \Sigma: x<\left(\phi_{X}^{\alpha}\right)^{-1}\left(T_{Y}^{\alpha}\right)\right\}, \mathcal{I}^{\alpha}=\left\{x \in \Sigma: x<T_{Y}^{\alpha}\right\}, \quad \text { if } \alpha<0 \text { and } \\
& \mathcal{D}^{\alpha}=\left\{x \in \Sigma: x<T_{X}^{\alpha}\right\}, \mathcal{I}^{\alpha}=\left\{x \in \Sigma: x<\left(\phi_{Y}^{\alpha}\right)\left(T_{X}^{\alpha}\right)\right\}, \text { if } \alpha>0 .
\end{aligned}
$$

Using the expressions given for $\phi_{X}^{\alpha}$ and $\phi_{Y}^{\alpha}$ in Proposition 2.12 applied to $X^{\alpha}$ and $Y^{\alpha}$, the return map is given by

$$
\phi^{\alpha}(x)=2\left(T_{Y}^{\alpha}-T_{X}^{\alpha}\right)+x-\beta_{X}\left(x-T_{X}^{\alpha}\right)^{2}+\beta_{Y}\left(2 T_{X}^{\alpha}-T_{Y}^{\alpha}-x\right)^{2}+O_{3}\left(x, T_{X}^{\alpha}, T_{Y}^{\alpha}\right)
$$

where $T_{X}^{\alpha}$ and $T_{Y}^{\alpha}$ are given in $(2.18)$

To look for periodic orbits near the fold-fold point, we look for zeros of the the auxiliary map

$$
\Phi:(\alpha, x) \in \mathcal{W} \subset\left(-\alpha_{0}, \alpha_{0}\right) \times \mathbb{R} \mapsto \Phi(\alpha, x)=\phi^{\alpha}(x)-x \in \mathbb{R} .
$$

The map $\Phi$ satisfies $\Phi(0,0)=0, \frac{\partial}{\partial x} \Phi(0,0)=0$ and $\frac{\partial^{2}}{\partial x^{2}} \Phi(0,0)=2 \mu_{Z} \neq 0$. Then, by the Implicit Function Theorem, for each $\alpha$ sufficiently small there exists a unique $C(\alpha)$ near $0 \in \Sigma$ such 

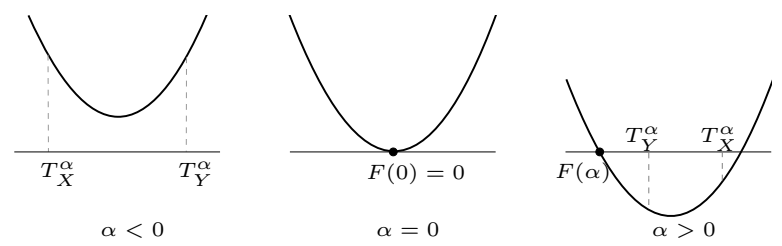

Figure 3: The graphic of $\Phi(\alpha, x)=\phi^{\alpha}(x)-x$ when $\mu_{Z}>0$, for different values of the parameter $\alpha$.

that $\frac{\partial}{\partial x} \Phi(\alpha, C(\alpha))=0$. Moreover, as $T_{X}^{\alpha}, T_{Y}^{\alpha}=O(\alpha)$ also $C(\alpha)=O(\alpha)$. Thus the map $\Phi(\alpha, x)$ has a critical point at $C(\alpha)$ which is a maximum or minimum depending on the sign of $\mu_{Z}$.

If $\mu_{Z}>0$ (see Figure 3) $C(\alpha)$ is a local minimum of $\Phi(\alpha,$.$) . If \alpha<0$, then $\Phi(\alpha, C(\alpha))=$ $2\left(T_{Y}^{\alpha}-T_{X}^{\alpha}\right)+O\left(\alpha^{2}\right)>0$, being $C(\alpha)$ a minimum this means that $\Phi(\alpha, x)>0$, for $x \in \Sigma$. Therefore there are no fixed points for $\phi^{\alpha}$ if $\alpha<0$. If $\alpha>0$ we obtain $\Phi(\alpha, C(\alpha))<0$ and therefore $\Phi(\alpha, x)$ has two zeros. Moreover, $\Phi\left(\alpha, T_{Y}^{\alpha}\right)<0$, and we call $F(\alpha) \in \mathcal{D}^{\alpha}$ the zero of $\Phi$ satisfying $F(\alpha)<T_{Y}^{\alpha}$. Therefore, the map $\phi^{\alpha}$ has a fixed point $F(\alpha)$ which corresponds to an attracting crossing cycle $\Gamma^{\alpha}$, since

$$
\frac{\partial}{\partial x} \phi^{\alpha}(F(\alpha))<1
$$

Summarizing the case $\mu_{Z}>0$ : for $\alpha<0$ the vector field $Z^{\alpha}$ has an stable pseudo-node $Q(\alpha)$ and no crossing cycles exist. When $\alpha>0$, the point $Q(\alpha)$ is a unstable pseudo-node and an attracting crossing cycle $\Gamma^{\alpha}$ through the point $(F(\alpha), 0)$ appears. Using that $T_{X}^{\alpha}$ and $T_{Y}^{\alpha}$ are $O(\alpha)$ one can compute

$$
F(\alpha)=\left(-\sqrt{\frac{2\left(T_{X}^{\alpha}-T_{Y}^{\alpha}\right)}{\mu_{Z}}}+\mathcal{O}(\alpha), 0\right) .
$$

When $\mu_{Z}<0$ the point $C(\alpha)$ is a local maximum of $\Phi(\alpha, x)$. Thus an repelling crossing cycle exists for $\alpha<0$ and no crossing cycles appear for $\alpha>0$. The nature of the pseudo-equilibrium $Q(\alpha)$ remains the same as in the case $\mu_{Z}<0$, since its stability does not depend on $\mu_{Z}$.

Then any unfolding of $Z \in \Lambda^{F}$ satisfying $(B), X^{1} \cdot Y^{1}(\mathbf{0})<0$ with $\mu_{Z} \neq 0$ leads to vector fields with exactly the same topological invariants, that is, the same $\Sigma$-regions and singularities; see Figures 4 and 5 .
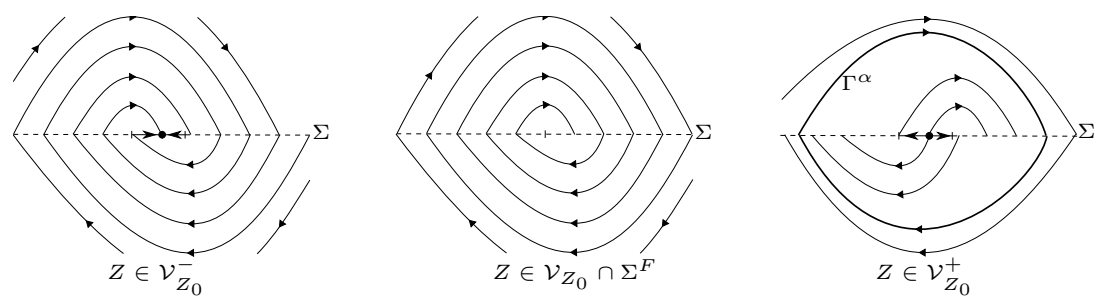

Figure 4: The unfolding of $Z \in \Lambda^{F}$ satisfying $(B), X^{1} \cdot Y^{1}(\mathbf{0})<0$ and $\mu_{Z}>0$.
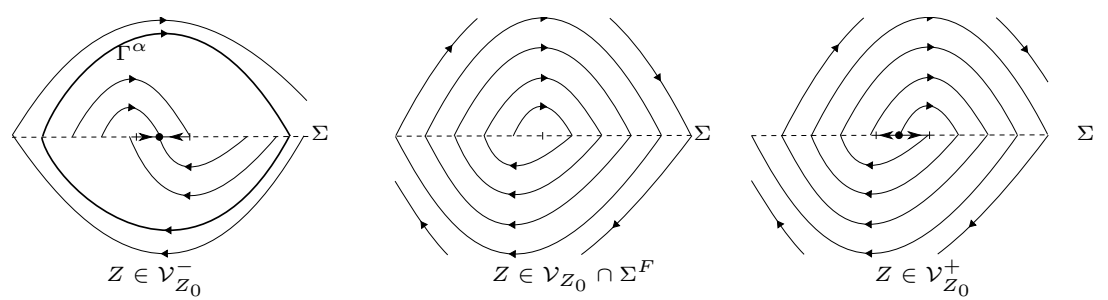

Figure 5: The unfolding of $Z \in \Lambda^{F}$ satisfying satisfying $(B), X^{1} \cdot Y^{1}(\mathbf{0})<0$ and $\mu_{Z}<0$.

Proposition 2.19. Let $Z \in \Lambda^{F}$ satisfying condition $(B)$ of Theorem 2.6, $X^{1} \cdot Y^{1}(\mathbf{0})>0$ and $\mu_{Z} \neq 0$. Let $\mathcal{V}_{0}$ be the neighborhood given in Proposition 2.15. Then any smooth curve

$$
\gamma: \alpha \in\left(-\alpha_{0}, \alpha_{0}\right) \mapsto Z^{\alpha} \in \mathcal{V}_{0}
$$


which is transverse to $\Lambda^{F}$ at $\gamma(0)=Z$ leads to the same behaviors in $\mathcal{V}_{0}^{+}$and in $\mathcal{V}_{0}^{-}$. For any $Z \in \mathcal{V}_{0}^{ \pm}$has two invisible folds with a crossing region between them. In both cases, the sliding vector field has no pseudo-equilibrium Moreover, in the case $\mu_{Z}>0$ then $Z \in \mathcal{V}_{0}^{+}$has an pseudocycle and when $\mu_{Z}<0$ then $Z \in \mathcal{V}_{0}^{-}$has a pseudo-cycle. Therefore, there exists a weak equivalence between any two unfoldings of $Z$.

Proof. Since $X^{1} \cdot Y^{1}(\mathbf{0})>0, \Sigma=\overline{\Sigma^{s} \cup \Sigma^{e}}$ for $\alpha=0$. For $\alpha \neq 0$, since for all points $(x, 0)$ between the folds $T_{X}^{\alpha}$ and $T_{Y}^{\alpha}$ the vector field $Z^{\alpha}$ satisfies $X^{\alpha 2} \cdot Y^{\alpha 2}(x, 0)>0$. Therefore, a crossing region appears between the folds. The discontinuity curve becomes $\Sigma=\overline{\Sigma^{e} \cup \Sigma^{c} \cup \Sigma^{s}}$, where

$$
\begin{aligned}
\Sigma^{e} & =\left\{x \in \Sigma: x<\min \left\{T_{X}^{\alpha}, T_{Y}^{\alpha}\right\}\right\}, \\
\Sigma^{c} & =\left\{x \in \Sigma: x \in\left(\min \left\{T_{X}^{\alpha}, T_{Y}^{\alpha}\right\}, \max \left\{T_{X}^{\alpha}, T_{Y}^{\alpha}\right\}\right)\right\}, \\
\Sigma^{s} & =\left\{x \in \Sigma: x>\max \left\{T_{X}^{\alpha}, T_{Y}^{\alpha}\right\}\right\} .
\end{aligned}
$$

Using the same argument as in Proposition 2.16, no pseudo-equilibrium appears for $\alpha \neq 0$. As mentioned in Remark 2.13, one needs to consider the generalized first return map for this case.

For convenience, we set

$$
\phi_{Z}^{\alpha}=\phi_{Y}^{\alpha} \circ \phi_{X}^{\alpha}=2\left(T_{Y}^{\alpha}-T_{X}^{\alpha}\right)+x-\beta_{X}\left(x-T_{X}^{\alpha}\right)^{2}+\beta_{Y}\left(2 T_{X}^{\alpha}-T_{Y}^{\alpha}-x\right)^{2}+O_{3}\left(x, T_{X}^{\alpha}, T_{Y}^{\alpha}\right)
$$

By the same arguments of Proposition 2.18 a pseudo-cycle appears for $\alpha>0$ when $\mu_{Z}>0$ and it appears for $\alpha<0$ when $\mu_{Z}<0$.

Then any unfolding of $Z \in \Lambda^{F}$ satisfying $(B), X^{1} \cdot Y^{1}(\mathbf{0})>0$ with $\mu_{Z} \neq 0$ leads to vector fields with exactly the same topological invariants, that is, the same $\Sigma$-regions and singularities; see Figures 6 and 7
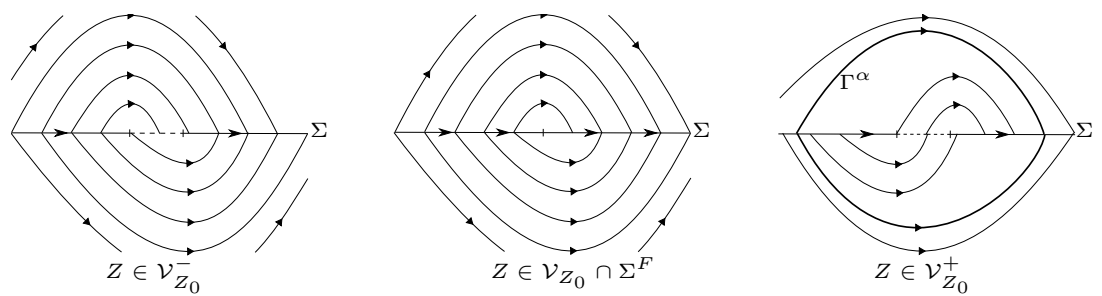

Figure 6: The unfolding of $Z \in \Lambda^{F}$ satisfying $(B), X^{1} \cdot Y^{1}(\mathbf{0})>0$ and $\mu_{Z}<0$.
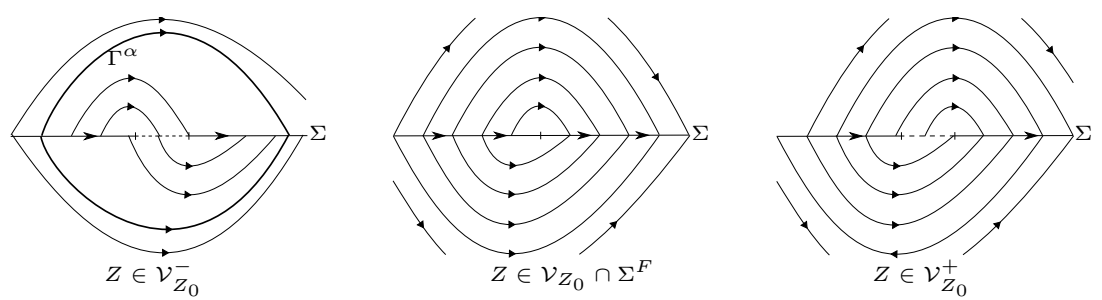

Figure 7: The unfolding of $Z \in \Lambda^{F}$ satisfying satisfying $(B), X^{1} \cdot Y^{1}(\mathbf{0})>0$ and $\mu_{Z}>0$.

\subsection{The versal unfolding of a visible-invisible fold-fold singularity}

This section is devoted to the study of the unfoldings of a Filippov vector field having a visibleinvisible fold point. We have essentially three different bifurcations, two of them occur when the vector fields $X$ and $Y$ point at opposite directions at the fold-fold point and differ in the sign of $(\operatorname{det} Z)_{x}(\mathbf{0})$. The third occurs when both vector fields point to the same direction.

Proposition 2.20. Let $Z \in \Lambda^{F}$ satisfying condition $(C)$ of Theorem 2.6 and $X^{1} \cdot Y^{1}(\mathbf{0})<0$. Let $\mathcal{V}_{0}$ be the neighborhood given in Proposition 2.15. Then any smooth curve

$$
\gamma: \alpha \in\left(-\alpha_{0}, \alpha_{0}\right) \mapsto Z^{\alpha} \in \mathcal{V}_{0}
$$


which is transverse to $\Lambda^{F}$ at $\gamma(0)=Z$ leads to the same behaviors in $\mathcal{V}_{0}^{+}$and in $\mathcal{V}_{0}^{-}$.

This behavior depends of the sign of $(\operatorname{det} Z)_{x}(\mathbf{0})$ :

1. If $(\operatorname{det} Z)_{x}(\mathbf{0})>0$ :

- Every $Z \in \mathcal{V}_{0}^{-}$has a visible and an invisible fold points and there exists a crossing region between them. The sliding vector field has a pseudo-saddle $Q(\alpha)=(x(\alpha), 0) \in \Sigma^{s}$ situated on "left" of both folds.

- Every $Z \in \mathcal{V}_{0}^{+}$has a visible and an invisible fold points and there exists a crossing region between them. The sliding vector field has a pseudo-saddle $Q(\alpha)=(x(\alpha), 0) \in \Sigma^{e}$ situated on the "right" of both folds.

2. If $(\operatorname{det} Z)_{x}(\mathbf{0})<0$ :

- Every $Z \in \mathcal{V}_{0}^{-}$has a visible and an invisible fold points and there exists a crossing region between them. The sliding vector field has a unstable pseudo-node $Q(\alpha)=(x(\alpha), 0) \in \Sigma^{e}$ situated on the "right" of both folds.

- Every $Z \in \mathcal{V}_{0}^{+}$has a visible and an invisible fold points and there exists a crossing region between them. The sliding vector field has a stable pseudo-node $Q(\alpha)=(x(\alpha), 0) \in \Sigma^{s}$ situated on "left" of both folds.

Therefore, there exists a weak equivalence between any two unfoldings of $Z$.

Proof. Since $X^{1} \cdot Y^{1}(\mathbf{0})<0, \Sigma=\overline{\Sigma^{s} \cup \Sigma^{e}}$ for $\alpha=0$. For $\alpha \neq 0$, for all points $(x, 0)$ between the folds $T_{X}^{\alpha}$ and $T_{Y}^{\alpha}$ the vector field $Z^{\alpha}$ satisfies $X^{\alpha 2} \cdot Y^{\alpha 2}(x, 0)>0$. Therefore, a piece of crossing region appears between the folds for $\alpha \neq 0$. The discontinuity curve becomes $\Sigma=\overline{\Sigma^{s} \cup \Sigma^{c} \cup \Sigma^{e}}$, where

$$
\begin{aligned}
& \Sigma^{s}=\left\{x \in \Sigma: x<\min \left\{T_{X}^{\alpha}, T_{Y}^{\alpha}\right\}\right\}, \\
& \Sigma^{c}=\left\{x \in \Sigma: x \in\left(\min \left\{T_{X}^{\alpha}, T_{Y}^{\alpha}\right\}, \max \left\{T_{X}^{\alpha}, T_{Y}^{\alpha}\right\}\right)\right\}, \\
& \Sigma^{e}=\left\{x \in \Sigma: x>\max \left\{T_{X}^{\alpha}, T_{Y}^{\alpha}\right\}\right\} .
\end{aligned}
$$

By condition $(C), \gamma \neq 0$ (see $(2.9)$ implying $(\operatorname{det} Z)_{x}(\mathbf{0}) \neq 0$, then Corolary 2.11 guarantees the existence of a unique point $Q(\alpha) \in \Sigma$ such that $\operatorname{det} Z^{\alpha}(Q(\alpha))=0$. To check if $Q(\alpha)$ belongs to $\Sigma^{s, e}$ and therefore it is a pseudo-equilibrium one must analyze separately the cases $(\operatorname{det} Z)_{x}(\mathbf{0})>0$ and $(\operatorname{det} Z)_{x}(\mathbf{0})<0$.

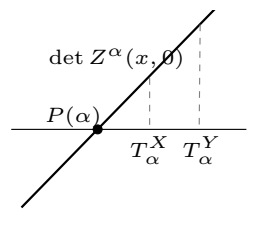

$\alpha<0$

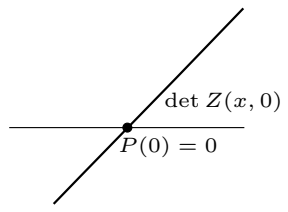

$\alpha=0$

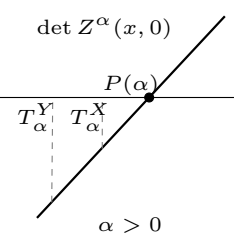

$\alpha>0$

Figure 8: The curve $\operatorname{det} Z^{\alpha}(x, 0)$ when $(\operatorname{det} Z)_{x}(\mathbf{0})$ for each $\alpha$.

Suppose that $(\operatorname{det} Z)_{x}(\mathbf{0})>0$, so for $|\alpha|<<1$ we have $(\operatorname{det} Z)_{x}^{\alpha}(x, 0)>0$ for $x \in \Sigma$. Therefore, the function $\operatorname{det} Z^{\alpha}: x \in \Sigma \mapsto \operatorname{det} Z^{\alpha}(x, 0) \in \mathbb{R}$ is increasing, see Figure 8 . Computing the values of $\operatorname{det} Z^{\alpha}\left(T_{X}^{\alpha}, 0\right)$ and $\operatorname{det} Z^{\alpha}\left(T_{Y}^{\alpha}, 0\right)$ we conclude that the pseudo-equilibrium $Q(\alpha)$ belongs to $\Sigma^{e}$ for $\alpha>0$ and it belongs to $\Sigma^{s}$ when $\alpha<0$. In addition, by $(2.4)$ we have that $\left(Z^{s}\right)^{\prime}(x(\alpha))>0$ if $\alpha<0$ and $\left(Z^{s}\right)^{\prime}(x(\alpha))<0$ if $\alpha>0$ and therefore the point $Q(\alpha)$ is a pseudo-saddle.

When $(\operatorname{det} Z)_{x}(\mathbf{0})<0$, the map $\operatorname{det} Z(x, 0)$ is decreasing. Therefore, by the same argument the pseudo-equilibrium belongs to $\Sigma^{s}$ if $\alpha>0$ and to $\Sigma^{e}$ if $\alpha<0$. In this case, computing $\left(Z^{s}\right)^{\prime}(x(\alpha))$ the point $Q(\alpha)$ is a pseudo-node.

Then any unfolding of $Z \in \Lambda^{F}$ satisfying $(C)$ with $X^{1} \cdot Y^{1}(\mathbf{0})<0$ leads to vector fields with exactly the same topological invariants, that is, the same $\Sigma$-regions and singularities, depending on the sign of $(\operatorname{det} Z)_{x}(\mathbf{0})$, see Figures 9 and 10 . 

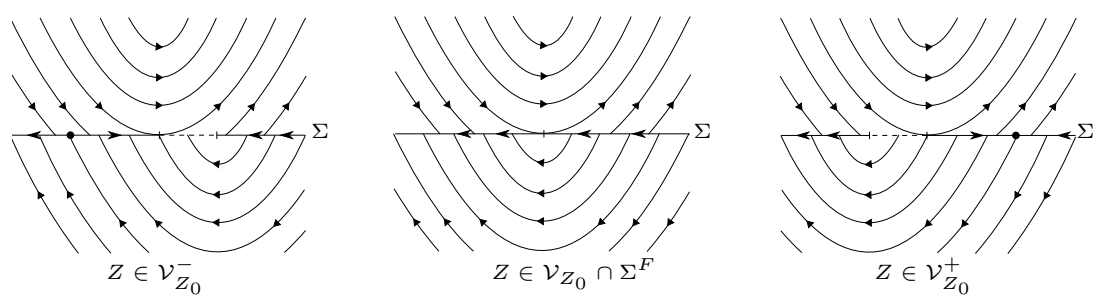

Figure 9: The unfolding of $Z \in \Lambda^{F}$ satisfying $(C), X^{1} \cdot Y^{1}(\mathbf{0})<0$ and $(\operatorname{det} Z)_{x}(\mathbf{0})>0$.
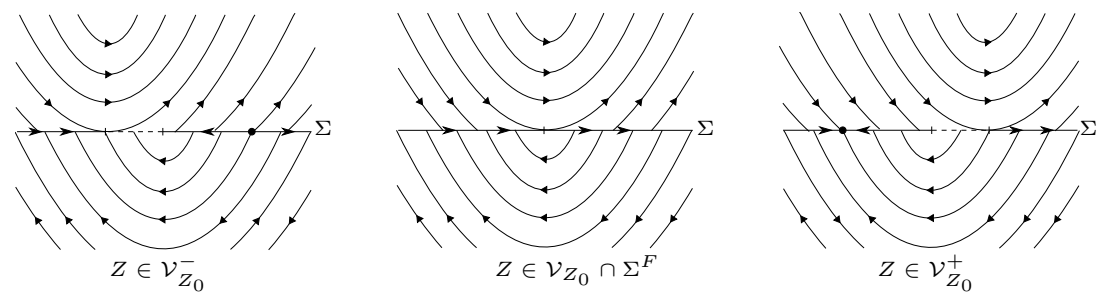

Figure 10: The unfolding of $Z \in \Lambda^{F}$ satisfying $(C), X^{1} \cdot Y^{1}(\mathbf{0})<0$ and $(\operatorname{det} Z)_{x}(\mathbf{0})<0$.

Proposition 2.21. Let $Z \in \Lambda^{F}$ satisfying condition $(C)$ of Figure 6 and $X^{1} \cdot Y^{1}(\mathbf{0})>0$. Let $\mathcal{V}_{0}$ be the neighborhood given in Proposition 2.15. Then any smooth curve

$$
\gamma: \alpha \in\left(-\alpha_{0}, \alpha_{0}\right) \mapsto Z^{\alpha} \in \mathcal{V}_{0}
$$

which is transverse to $\Lambda^{F}$ at $\gamma(0)=Z$ leads to the same behaviors in $\mathcal{V}_{0}^{+}$and in $\mathcal{V}_{0}^{-}$.

Every $Z \in \mathcal{V}_{0}^{-}$has one visible and one invisible fold points and there exists a region of escaping between them. The sliding vector field has no pseudo equilibrium.

Every $Z \in \mathcal{V}_{0}^{+}$has one visible and one invisible fold points and there exists a region of sliding between them. The sliding vector field has no pseudo equilibrium.

Therefore, there exists a weak equivalence between any two unfoldings of $Z$.

Proof. Since $X^{1} \cdot Y^{1}(\mathbf{0})>0, \Sigma=\overline{\Sigma^{c}}$ for $\alpha=0$. For $\alpha \neq 0$, for all points $(x, 0)$ between the folds $T_{X}^{\alpha}$ and $T_{Y}^{\alpha}$ the vector field $Z^{\alpha}$ satisfies $X^{\alpha 2} \cdot Y^{\alpha 2}(x, 0)<0$. Therefore, a piece of sliding $(\alpha>0)$ or escaping $(\alpha<0)$ region appears between the folds for $\alpha \neq 0$. The discontinuity curve becomes $\Sigma=\overline{\Sigma^{c} \cup \Sigma^{e, s}}$, where

$$
\begin{aligned}
\Sigma^{c} & =\left\{x \in \Sigma: x<\min \left\{T_{X}^{\alpha}, T_{Y}^{\alpha}\right\}\right\} \cup\left\{x \in \Sigma: x>\max \left\{T_{X}^{\alpha}, T_{Y}^{\alpha}\right\}\right\} \\
\Sigma \backslash \Sigma^{c} & = \begin{cases}\Sigma^{s}= & \left.\left\{x \in \Sigma: x \in\left(T_{Y}^{\alpha}, T_{X}^{\alpha}\right)\right\}\right\}, \alpha>0 \\
\Sigma^{e}= & \left.\left\{x \in \Sigma: x \in\left(T_{X}^{\alpha}, T_{Y}^{\alpha}\right)\right\}\right\}, \alpha<0\end{cases}
\end{aligned}
$$

A simple computation shows that if $\alpha>0$ then $\operatorname{det} Z^{\alpha}(x, 0)>0$ for all $x \in\left[T_{Y}^{\alpha}, T_{X}^{\alpha}\right]$, therefore $\left(Z^{\alpha}\right)^{s}(x)>0$ in the sliding region. Analogously, for $\alpha<0$ we have $\operatorname{det} Z^{\alpha}(x, 0)<0$ for all $x \in\left[T_{X}^{\alpha}, T_{Y}^{\alpha}\right]$, therefore $\left(Z^{\alpha}\right)^{s}(x)>0$ in the escaping region.
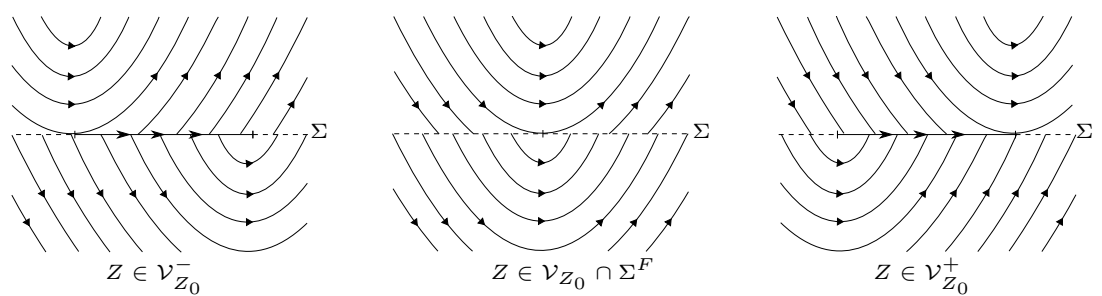

Figure 11: The unfolding of $Z \in \Lambda^{F}$ satisfying $(C)$ and $X^{1} \cdot Y^{1}(\mathbf{0})>0$.

Then any unfolding of $Z \in \Lambda^{F}$ satisfying $(C)$ and $X^{1} \cdot Y^{1}(\mathbf{0})>0$ leads to vector fields with exactly the behavior. Therefore, there exist a weak equivalence between the unfoldings of $Z_{0}$.

Joining the results obtained in Proposition 2.15 and in Propositions 2.16 to 2.21 we prove Theorem 2.6 stated at the beginning of this section. 


\section{The regularization near some generic codimension one fold-fold singularity}

In this section we study the regularization of the versal unfolding $Z^{\alpha}$ of $Z \in \Lambda^{F}$ studied in section 2. We will work with the Sotomayor-Teixeira regularization (see [ST98]) which is the vector field $Z_{\varepsilon}^{\alpha}$ given by

$$
Z_{\varepsilon}^{\alpha}(x, y)=\frac{1}{2}\left[\left(X^{\alpha}+Y^{\alpha}\right)(x, y)+\varphi\left(\frac{y}{\varepsilon}\right)\left(X^{\alpha}-Y^{\alpha}\right)(x, y)\right]
$$

where $\varphi$ is any sufficiently smooth transition function satisfying

$$
\varphi(v)=\left\{\begin{array}{ll}
1, & v \geq 1 \\
-1, & v \leq-1
\end{array} \text { and } \varphi^{\prime}(v)>0 \text { for } v \in(-1,1) .\right.
$$

Observe that, rescaling time $t \rightarrow 2 t$, the vector field (3.1) gives rise to the differential equations

$$
\left\{\begin{array}{l}
\dot{x}=G^{1}(x, y ; \alpha, \varepsilon) \\
\dot{y}=G^{2}(x, y ; \alpha, \varepsilon)
\end{array},\right.
$$

where

$$
G^{i}(x, y ; \alpha, \varepsilon)=\left(X^{\alpha i}+Y^{\alpha i}\right)(x, y)+\varphi\left(\frac{y}{\varepsilon}\right)\left(X^{\alpha i}-Y^{\alpha i}\right)(x, y), i=1,2 .
$$

Performing the change $y=\varepsilon \cdot v$ in (3.3) we obtain the so called slow system $\bar{Z}_{\varepsilon}^{\alpha}$ :

$$
\left\{\begin{array}{l}
\dot{x}=F^{1}(x, v ; \alpha, \varepsilon) \\
\varepsilon \dot{v}=F^{2}(x, v ; \alpha, \varepsilon)
\end{array}\right.
$$

where

$$
F^{i}(x, v ; \alpha, \varepsilon)=\left(X^{\alpha i}+Y^{\alpha i}\right)(x, \varepsilon v)+\varphi(v)\left(X^{\alpha i}-Y^{\alpha i}\right)(x, \varepsilon v), i=1,2 .
$$

After the change of time $\tau=\frac{t}{\varepsilon}$, system (3.5) becomes the so called fast system, which is a smooth vector field $\tilde{Z}_{\varepsilon}^{\alpha}$ depending on two parameters $(\alpha, \varepsilon)$ :

$$
\left\{\begin{array}{l}
x^{\prime}=\varepsilon F^{1}(x, v ; \alpha, \varepsilon) \\
v^{\prime}=F^{2}(x, v ; \alpha, \varepsilon)
\end{array}\right.
$$

Remark 3.1. Even if systems (3.5) and (3.7) are formally slow-fast systems, when $|v| \geq 1$ these systems are the original smooth vector fields $X$ and $Y$ written in variables $(x, v)=\left(x, \frac{y}{\varepsilon}\right)$. In particular, the existence of "big periodic orbits" in section 4.3 will be a consequence of the slow-fast nature of these systems for $|v| \leq 1$ combined with the behavior of the original systems $X$ and $Y$ for $v \geq 1$ and $v \leq-1$ respectively.

Remark 3.2. Since one can write

$$
Z^{\alpha}=Z+\tilde{Z} \alpha+\mathcal{O}\left(\alpha^{2}\right), \quad Z=(X, Y), \quad \tilde{Z}=(\tilde{X}, \tilde{Y}),
$$

the regularized system can be written as $Z_{\varepsilon}^{\alpha}=Z_{\varepsilon}+\tilde{Z}_{\varepsilon} \alpha+\mathcal{O}\left(\alpha^{2}\right)$, where $Z_{\varepsilon}$ and $\tilde{Z}_{\varepsilon}$ are the $\varphi$-regularization of $Z$ and $\tilde{Z}$, respectively.

\subsection{Critical points of the regularized system $Z_{\varepsilon}^{\alpha}$}

To understand the dynamics of $Z_{\varepsilon}^{\alpha}$ we begin by studying its equilibrium points.

Lemma 3.3. There exist $\alpha_{0}, \varepsilon_{0}>0$ such that for every $-\alpha_{0}<\alpha<\alpha_{0}$ and $0<\varepsilon<\varepsilon_{0}$ one has:

(a) If $X^{1} \cdot Y^{1}(\mathbf{0})>0, Z_{\varepsilon}^{\alpha}$ has no critical points; 
(b) If $X^{1} \cdot Y^{1}(\mathbf{0})<0, Z_{\varepsilon}^{\alpha}$ has a unique critical point:

$$
P(\alpha, \varepsilon)=(x(\alpha, \varepsilon), \varepsilon v(\alpha, \varepsilon))=Q(\alpha)+\mathcal{O}(\varepsilon)=(\bar{x}, 0) \alpha+\left(x^{*}, v^{*}\right) \varepsilon+\mathcal{O}_{2}(\alpha, \varepsilon),
$$

where $Q(\alpha)$ is the pseudo-equilibrium of $Z^{\alpha}$, and $v^{*}, x^{*}$ and $\bar{x}$ are given in (3.12), (3.13) and (3.14).

Proof. Using the change $y=\varepsilon v$, we look for zeros of the map

$$
F(x, v ; \alpha, \varepsilon)=\left(F^{1}(x, v ; \alpha, \varepsilon), F^{2}(x, v ; \alpha, \varepsilon)\right) .
$$

At first we consider $F^{1}(x, v ; \alpha, 0)=0$, which is solvable if and only if for each $x$ there exists $v(x) \in(-1,1)$ satisfying

$$
\varphi(v(x))=-\frac{X^{\alpha 1}+Y^{\alpha 1}}{X^{\alpha 1}-Y^{\alpha 1}}(x, 0)
$$

If $X^{1} \cdot Y^{1}(\mathbf{0})>0$ then Equation (3.11) has no solution for $\alpha$ small enough, since the absolute value of the right-hand side of equation $[(3.12)$ is greater than one. Therefore, by continuity the vector field $Z_{\varepsilon}^{\alpha}$ has no critical points near the origin, for $\varepsilon>0$ sufficiently small.

If $X^{1} \cdot Y^{1}(\mathbf{0})<0$ for $\alpha$ small enough the absolute value of the right-hand side of Equation (3.11) is smaller than one, then for each $x$ it admits a solution $v(x) \in(-1,1)$. Moreover,

$$
F^{2}(x, v(x) ; \alpha, 0)=-\frac{2 \operatorname{det} Z^{\alpha}}{Y^{\alpha 1}-X^{\alpha 1}}(x, 0) .
$$

By Corolary 2.11, applied to the vector field $Z^{\alpha}$, for small $\alpha$ there exists a unique solution $x(\alpha)=P\left(Z^{\alpha}\right)$ near the origin such that $\left.\operatorname{det} Z^{\alpha}(x(\alpha), 0)\right)=0$ and $\operatorname{sgn}\left(\left(\operatorname{det} Z^{\alpha}\right)_{x}(x(\alpha), 0)\right)=$ $\operatorname{sgn}\left((\operatorname{det} Z)_{x}(\mathbf{0})\right)$. Therefore, setting $v(\alpha)=v(x(\alpha))$ we have $F(x(\alpha), v(\alpha) ; \alpha, 0)=0$. Moreover, a straightforward computation shows that

$$
\operatorname{det} D_{(x, v)} F(x(\alpha), v(\alpha) ; \alpha, 0)=-2 \varphi^{\prime}(v(\alpha))\left(\operatorname{det} Z^{\alpha}\right)_{x}(x(\alpha), 0) \neq 0
$$

By applying the Implicit Function Theorem we obtain that $Z_{\varepsilon}^{\alpha}$ has a critical point $P(\alpha, \varepsilon)=$ $(x(\alpha), 0)+\mathcal{O}(\varepsilon)$ for $\varepsilon>0$ sufficiently small. Moreover, by the Chain's Rule we get the expressions for $v^{*}, x^{*}$ and $\bar{x}$ : using the notation given in (3.8)

$$
\begin{aligned}
v^{*} & =\varphi^{-1}\left(-\frac{X^{1}+Y^{1}}{X^{1}-Y^{1}}(\mathbf{0})\right) \\
x^{*} & =-\frac{(\operatorname{det} Z)_{y}}{(\operatorname{det} Z)_{x}}(\mathbf{0}) v^{*} \\
\bar{x} & =\frac{Y^{1} \tilde{X}^{2}-X^{1} \tilde{Y}^{2}}{(\operatorname{det} Z)_{x}}(\mathbf{0}) .
\end{aligned}
$$

Observe that for $\alpha \neq 0$, the point $Q(\alpha)=(x(\alpha), 0)$ is the pseudo-equilibrium of the sliding vector field which appears in the unfolding $Z^{\alpha}$. That is, the critical point $P(\alpha, \varepsilon)$ that arises after the regularization derives from the pseudo-equilibrium of $Z^{\alpha}$. Moreover $x(\alpha)=\bar{x} \alpha+\mathcal{O}\left(\alpha^{2}\right)$.

Remark 3.4. Observe that when $\alpha$ tends to zero, even though the pseudo-equilibrium $Q(\alpha)$ for $Z_{\alpha}^{s}$ disappears and becomes the fold-fold point of $Z$, the critical point $P(\alpha, \varepsilon)$ of $Z_{\varepsilon}^{\alpha}$ tends to $P(0, \varepsilon)$ which is the critical point of $Z_{\varepsilon}$.

To obtain the topological character of the critical point $P(\alpha, \varepsilon)$ we need information about the determinant and the trace of $D Z_{\varepsilon}^{\alpha}(P(\alpha, \varepsilon))$.

Proposition 3.5. Consider $Z \in \Lambda^{F}$ and $X^{1} \cdot Y^{1}(\mathbf{0})<0$. Then at the critical point $P(\alpha, \varepsilon)$ we have 
(a) $\operatorname{det} D Z_{\varepsilon}^{\alpha}(P(\alpha, \varepsilon))=-\frac{1}{\varepsilon}\left(2 \varphi^{\prime}(v(\alpha))\left(\operatorname{det} Z^{\alpha}\right)_{x}(x(\alpha), 0)+\mathcal{O}(\varepsilon)\right)$;

(b) $\operatorname{tr} D Z_{\varepsilon}^{\alpha}(P(\alpha, \varepsilon))=\frac{1}{\varepsilon}\left(\varphi^{\prime}(v(\alpha))\left(X^{\alpha 2}-Y^{\alpha 2}\right)(x(\alpha), 0)+\mathcal{O}(\varepsilon)\right)$, for $\alpha \neq 0$.

\section{Moreover}

(c) $\operatorname{det} D Z_{\varepsilon}^{\alpha}(P(\alpha, \varepsilon))=-\frac{1}{\varepsilon}\left(2 \varphi^{\prime}\left(v^{*}\right)(\operatorname{det} Z)_{x}(\mathbf{0})+\mathcal{O}(\alpha, \varepsilon)\right)$;

(d) $\operatorname{tr} D Z_{\varepsilon}^{\alpha}(P(\alpha, \varepsilon))=\frac{1}{\varepsilon}\left(N(Z, \tilde{Z}) \alpha+M(Z) \varepsilon+\mathcal{O}_{2}(\alpha, \varepsilon)\right)$,

where $M(Z)$ and $N(Z, \tilde{Z})$ are constants given by

$$
\begin{aligned}
M(Z) & =\left[\left(X_{x}^{1}+Y_{x}^{1}\right)+\varphi\left(v^{*}\right)\left(X_{x}^{1}-Y_{x}^{1}\right)+\left(X_{y}^{2}+Y_{y}^{2}\right)+\varphi\left(v^{*}\right)\left(X_{y}^{2}-Y_{y}^{2}\right)\right. \\
& \left.+\varphi^{\prime}\left(v^{*}\right)\left(\left(X_{x}^{2}-Y_{x}^{2}\right) x^{*}+\left(X_{y}^{2}-Y_{y}^{2}\right) v^{*}\right)\right](\mathbf{0}) \\
N(Z, \tilde{Z}) & =\frac{1}{(\operatorname{det} Z)_{x}(\mathbf{0})} \varphi^{\prime}\left(v^{*}\right)\left(X^{1}-Y^{1}\right)(\mathbf{0})\left(Y_{x}^{2} \tilde{X}^{2}-X_{x}^{2} \tilde{Y}^{2}\right)(\mathbf{0})
\end{aligned}
$$

with $v^{*}, x^{*}$ given in (3.12) and (3.13) respectively.

Proof. The proof can be seen in Lar15.

Remark 3.6. Since $\varphi^{\prime}\left(v^{*}\right),\left(X^{1}-Y^{1}\right)(\mathbf{0}) \neq 0$ and the transversality condition (2.19) guarantees that $\left(Y_{x}^{2} \tilde{X}^{2}-X_{x}^{2} \tilde{Y}^{2}\right)(\mathbf{0}) \neq 0$, the constant $N(Z, \tilde{Z}) \neq 0$ for any versal unfolding of $Z \in \Lambda^{F}$.

Proposition 3.7. Let $Z \in \Lambda^{F}$ satisfying $X^{1} \cdot Y^{1}(\mathbf{0})<0$ and $P(\alpha, \varepsilon)$ be the critical point of $Z_{\varepsilon}^{\alpha}$ given in Lemma 3.3. It follows that for $\alpha$ and $\varepsilon>0$ small enough:

(a) If $(\operatorname{det} Z)_{x}(\mathbf{0})>0$ the critical point $P(\alpha, \varepsilon)$ is a saddle;

(b) If $(\operatorname{det} Z)_{x}(\mathbf{0})<0$ the critical point $P(\alpha, \varepsilon)$ is a node or a focus. Moreover,:

(b1) There exist a curve $\mathcal{D}$ in the parameter plane $(\alpha, \varepsilon)$, given by

$$
\mathcal{D}=\left\{(\alpha, \varepsilon): \varepsilon=-\frac{N(Z, \tilde{Z})^{2}}{\left.8 \varphi^{\prime}\left(v^{*}\right)(\operatorname{det} Z)_{x}(\mathbf{0})\right)} \alpha^{2}+\mathcal{O}\left(\alpha^{3}\right)\right\},
$$

such that:

(i) For $(\alpha, \varepsilon)$ bellow the curve $\mathcal{D}$ the critical point $P(\alpha, \varepsilon)$ is a node;

(ii) For $(\alpha, \varepsilon)$ on the curve $\mathcal{D}$ the critical point $P(\alpha, \varepsilon)$ is a degenerate node;

(iii) For $(\alpha, \varepsilon)$ above the curve $\mathcal{D}$ the critical point $P(\alpha, \varepsilon)$ is a focus;

(b2) There exists a curve $\mathcal{H}$ in the parameter plane $(\alpha, \varepsilon)$, given by

$$
\mathcal{H}=\left\{(\alpha, \varepsilon): \alpha=\delta_{\mathcal{H}} \varepsilon+\mathcal{O}\left(\varepsilon^{2}\right)\right\}, \quad \delta_{\mathcal{H}}=-\frac{M(Z)}{N(Z, \tilde{Z})} .
$$

such that the critical point $P(\alpha, \varepsilon)$ undergoes a Hopf Bifurcation.

Proof. The proof can be seen in Lar15.

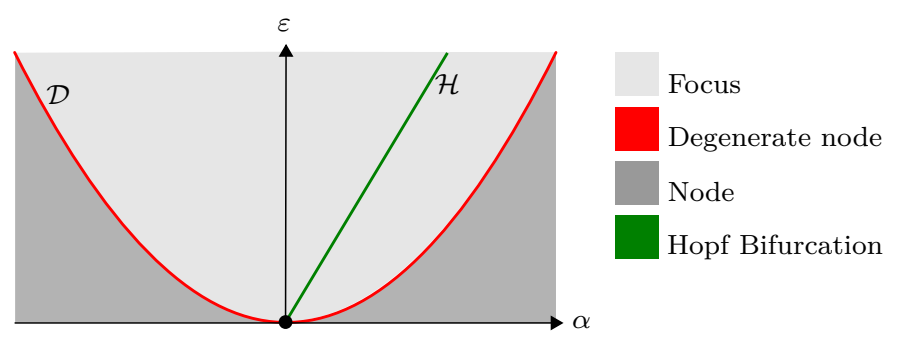

Figure 12: Topological type of the critical point $P(\alpha, \varepsilon)$ depending on each region of the $(\alpha, \varepsilon)$-parameter semi-plane. 
Corolary 3.8. Let $Z \in \Lambda^{F}$ satisfying $X^{1} \cdot Y^{1}(\mathbf{0})<0$. One has that

(a) if both folds are visible the critical point $P(\alpha, \varepsilon)$ is a saddle for $|\alpha|$ and $\varepsilon>0$ small enough;

(b) if both folds are invisible, the topological type of the critical point $P(\alpha, \varepsilon)$ changes as described in item (b) of Proposition 3.7. Moreover, the critical point $P(\alpha, \varepsilon)$ is stable for $(\alpha, \varepsilon)$ on the left of the curve $\mathcal{H}$ and it is unstable for $(\alpha, \varepsilon)$ on the right of the curve $\mathcal{H}$;

(c) if both folds have opposite visibility, then

(c1) if $(\operatorname{det} Z)_{x}(\mathbf{0})>0, P(\alpha, \varepsilon)$ is a saddle for every $|\alpha|$ and $\varepsilon>0$ small enough;

(c2) if $(\operatorname{det} Z)_{x}(\mathbf{0})<0$, the topological type of the critical point $P(\alpha, \varepsilon)$ changes as described in item (b) of Proposition 3.7. Moreover, the critical point $P(\alpha, \varepsilon)$ is unstable for $(\alpha, \varepsilon)$ on the left of the curve $\mathcal{H}$ and it is stable for $(\alpha, \varepsilon)$ on the right of the curve $\mathcal{H}$;

Proof. The proof is a consequence of Lemma 2.9 and Propositions 3.5 and 3.7 .

Remark 3.9. Over the curve $\mathcal{H}$ the character of the Hopf bifurcation is determined by the first Lyapunov coefficient $\ell_{1}$, see [HG83]. If $\ell_{1}>0$ the bifurcation is subcritical and gives rise to the existence of an unstable periodic orbit. If $\ell_{1}<0$ a stable periodic orbit arises at the Hopf bifurcation. However, the computation of $\ell_{1}$ leads to a cumbersome expression which does not add any relevant information. The only important thing is that it sign depends on the vector field $Z$ but also of the regularization function $\varphi$ as was already observed in [KH15b], where a formula for this coefficient for some suitable normal forms of $Z^{\alpha}$ was given (see formula (7.15) of that paper). In section 5 we will relate this coefficient with the derivative of a suitable Melnikov function at the point $\left(0, v^{*}\right)$ (see Proposition 5.1).

Remark 3.10. It is worth to mention that, when the non-smooth vector field $Z$ has an invisibleinvisible fold, the stability of the critical point $P(0, \varepsilon)$ of $Z_{\varepsilon}$ is not related with the stability of the fold-fold given by the first return map (2.12). Let us recall that the stability of the fold-fold point depends of $\mu_{Z}$ given in (2.15) and the stability of the focus depends of the sign of $\operatorname{tr} D Z_{\varepsilon}(P(0, \varepsilon))$ given in 3.5. Due to the cumbersome expression of these coefficients one could think that it is possible to relate the sign of both quantities but we will see in Example 3.1 that the signs of these coefficients are totally independent.

In fact, this is not surprising because the fold-fold point is a linear center for the return map $\phi_{Z}$ and its stability can be changed by a small perturbation.

Example 3.1 (From an attractive invisible fold-fold in the non-smooth vector field to a focus and a "linear" center in its regularization). Consider the one parameter family $Z_{\eta}=\left(X_{\eta}, Y\right)$ where

$$
\left\{\begin{array}{l}
X_{\eta}(x, y)=(-1+\eta x, x) \\
Y(x, y)=\left(1,2 x+x^{2}\right)
\end{array}\right.
$$

Observe that $Z_{\eta}$ is not a versal unfolding of $Z_{0}$, since we have an invisible fold-fold at the origin for all values of $\eta$. By Proposition 2.12, the return map associated to this family is given by

$$
\phi_{\eta}(x)=x+\frac{1}{3}(1-2 \eta) x^{2}-\frac{5}{9} x^{3}+\mathcal{O}\left(x^{4}\right) .
$$

For every $\eta<\frac{1}{2}, \mu_{Z}=\frac{1}{3}(1-2 \eta)>0$ and therefore the origin is an stable fixed point for the return map $\phi_{\eta}$ in (2.12).

Using a smooth transition function $\varphi$ as in Equation (3.2), the regularized system reads

$$
Z_{\varepsilon}^{\eta}(x, y)=\left\{\begin{array}{l}
\dot{x}=\left[\eta x+\varphi\left(\frac{y}{\varepsilon}\right)(-2+\eta x)\right], \\
\dot{y}=\left[3 x+x^{2}+\varphi\left(\frac{y}{\varepsilon}\right)\left(-x-x^{2}\right)\right] .
\end{array}\right.
$$


The critical point for this system is point $P(\varepsilon)=(0, \varepsilon v *)$ where $\varphi\left(v^{*}\right)=0$. At $P(\varepsilon)$ we have

$$
D Z_{\varepsilon}^{\alpha}(P(\varepsilon))=\left(\begin{array}{cc}
\eta & -2 \frac{1}{\varepsilon} \varphi^{\prime}\left(v^{*}\right) \\
3 & 0
\end{array}\right) \Rightarrow\left\{\begin{array}{l}
\operatorname{det} D Z_{\varepsilon}^{\eta}(P(\varepsilon))=\frac{6}{\varepsilon} \varphi^{\prime}\left(v^{*}\right)>0 \\
\operatorname{tr} D Z_{\varepsilon}^{\eta}(P(\varepsilon))=\eta
\end{array} .\right.
$$

It directly follows that the origin is an stable focus for $\eta<0$, a linear center for $\eta=0$ and an unstable focus for $\eta>0$.

Observe that $Z_{\eta}$ suffers a codimension two bifurcation when $\eta=\frac{1}{2}$, without moving the tangencies apart. By varying $\eta$ around $\eta=\frac{1}{2}$, the fold-fold changes its stability but $P(\varepsilon)$ remains an unstable focus.

\subsection{Critical manifolds of the regularized system $Z_{\varepsilon}^{\alpha}$}

In this section we will study the critical manifolds the slow-fast systems (3.5) and (3.7) . Setting $\varepsilon=0$ in $(3.5)$, the critical manifold $\Lambda_{0}^{\alpha}$ is given by

$$
\Lambda_{0}^{\alpha}=\left\{(x, v): F^{2}(x, v ; \alpha, 0)=\left(X^{\alpha 2}+Y^{\alpha 2}\right)(x, 0)+\varphi(v)\left(X^{\alpha 2}-Y^{\alpha 2}\right)(x, 0)=0\right\} .
$$

If $(x, 0) \in \Sigma^{c}, F^{2}(x, v ; \alpha, 0) \neq 0$ and therefore the critical manifold is not defined. If $(x, 0) \in \Sigma^{e, s}$ the equality

$$
\varphi(v)=\frac{\left(X^{\alpha 2}+Y^{\alpha 2}\right)(x, 0)}{\left(Y^{\alpha 2}-X^{\alpha 2}\right)(x, 0)}
$$

is well defined and it is solvable. Therefore the critical manifold is given by

$$
\Lambda_{0}^{\alpha}=\left\{(x, v): v=m_{0}^{\alpha}(x), x \in \Sigma^{e} \cup \Sigma^{s}\right\} .
$$

where

$$
m_{0}^{\alpha}(x)=\varphi^{-1}\left(\frac{X^{\alpha 2}+Y^{\alpha 2}}{Y^{\alpha 2}-X^{\alpha 2}}(x, 0)\right) .
$$

Observe that, for $\alpha \neq 0$, we have: $m_{0}^{\alpha}\left(T_{X}^{\alpha}\right)=1, m_{0}^{\alpha}\left(T_{Y}^{\alpha}\right)=-1$, where $\left(T_{X}^{\alpha}, 0\right)$ and $\left(T_{Y}^{\alpha}, 0\right)$ are the fold points of the vector fields $X^{\alpha}$ and $Y^{\alpha}$ given in (2.18). Moreover,

$$
\frac{d}{d x} m_{0}^{\alpha}(x)=\frac{d}{d x}\left(\varphi^{-1}\left(\frac{X^{\alpha 2}+Y^{\alpha 2}}{Y^{\alpha 2}-X^{\alpha 2}}(x, 0)\right)\right)=K^{\alpha}(x, 0) \cdot\left(\left(X^{\alpha 2}\right)_{x} Y^{\alpha 2}-\left(Y^{\alpha 2}\right)_{x} X^{\alpha 2}\right)(x, 0)
$$

where $K^{\alpha}(x, 0)=\frac{1}{\varphi^{\prime}\left(m_{0}^{\alpha}(x)\right)\left(\left(X^{\alpha}-Y^{\alpha}\right)(x, 0)\right)^{2}}>0$ for $\alpha \neq 0$. As $\varphi^{\prime}( \pm 1)=0$, when $x$ tends to the tangency points we have $\lim \frac{d}{d x} m_{0}^{\alpha}(x)= \pm \infty$, therefore $\Lambda_{0}^{\alpha}$ reaches $v= \pm 1$ at these points vertically.

The stability of the critical manifold $\Lambda_{0}^{\alpha}$ for system (3.7) is given by

$$
\left.\frac{\partial}{\partial v} F^{2}(x, v ; \alpha, \varepsilon)\right|_{\varepsilon=0}=\varphi^{\prime}(v)\left(X^{\alpha 2}-Y^{\alpha 2}\right)(x, 0),
$$

thus the critical manifold is repelling if it is defined over a escaping region and attracting if it is defined over a sliding region, see [ST98.

As it was seen in [ST98], the dynamics induced over the critical manifold $\Lambda_{0}^{\alpha}$ is equivalent to the dynamics of the sliding vector field $\left(Z^{\alpha}\right)^{s}$, defined in $\Sigma^{e} \cup \Sigma^{s}$, since by a simple computation and (2.2) we obtain

$$
\dot{x}=\left.F^{1}(x, v ; \alpha, 0)\right|_{\Lambda_{0}^{\alpha}}=2\left(Z^{\alpha}\right)^{s}(x) .
$$

Therefore, if the sliding vector field $\left(Z^{\alpha}\right)^{s}$ has a pseudo-equilibrium $Q(\alpha)=(x(\alpha), 0)$, the induced dynamics in $\Lambda_{0}^{\alpha}$ has a critical point at $\left(x(\alpha), m_{0}^{\alpha}(x(\alpha))\right.$ which has the same stability as the pseudoequilibrium $Q(\alpha)$. 
For $\alpha=0$, since the origin is a fold-fold point, one can write $F^{2}(x, v ; 0,0)=x \cdot \mathcal{A}(x, v)$ where

$$
\mathcal{A}(x, v)=(1+\varphi(v)) X_{x}^{2}(\mathbf{0})+(1-\varphi(v)) Y_{x}^{2}(\mathbf{0})+\mathcal{O}(x) .
$$

Therefore, in this case, the critical manifold $\Lambda_{0}^{0}$ decomposes as $\Lambda_{0}^{0}=C_{0} \cup \Lambda_{0}$, where

$$
C_{0}=\{(x, v): x=0\} \text { and } \Lambda_{0}=\{(x, v): \mathcal{A}(x, v)=0\} .
$$

Moreover, at $C_{0},(3.23)$ is identically zero, therefore $C_{0}$ is not a hyperbolic critical manifold of system (3.7) We will see in sections 3.2.1 and 3.2.2 that the critical manifold $\Lambda_{0}$ can be empty depending on the folds visibility.

During the rest of this paper we will restrict ourselves to the study of the regularization of $Z^{\alpha}$ in the case that $\left(X^{1} \cdot Y^{1}\right)(\mathbf{0})<0$. The dynamics of the other case is straightforward and can be found in $\mathrm{KH} 15 \mathrm{~b}$.

\subsubsection{Folds with the same visibility}

When the folds have the same visibility, for $\alpha=0$ Definition 2.3 implies that $X_{x}^{2} \cdot Y_{x}^{2}(\mathbf{0})>0$ and hence $\mathcal{A}(x, v) \neq 0$ for $(x, v)$ in a neighborhood of the origin, therefore $\Lambda_{0}=\{(x, v): \mathcal{A}(x, v)=$ $0\}=\varnothing$. The critical manifold is $\Lambda_{0}^{0}=C_{0}$ and it is not hyperbolic, see Fig. 13(a)

We saw in Propositions 2.17 and 2.18 that, for $\alpha \neq 0$, an sliding or escaping region appears between the two fold points. Therefore the critical manifold $\Lambda_{0}^{\alpha}$, given in $(3.20)$, is a smooth curve connecting the points $\left(T_{X}^{\alpha}, 1\right)$ and $\left(T_{Y}^{\alpha},-1\right)$. In addition, using Definition 2.3 and (3.22), we obtain that $\Lambda_{0}^{\alpha}$ is an increasing curve if $\alpha>0$ and decreasing if $\alpha<0$. Adding the results about the sliding and escaping regions of sections 2.1 and 2.2 we obtain:

- In the visible-visible case, see Fig. 13(a)

- If $\alpha<0, \Lambda_{0}^{\alpha}=\Lambda_{0}^{\alpha, u}$ is a decreasing curve connecting the points $\left(T_{X}^{\alpha}, 1\right)$ and $\left(T_{Y}^{\alpha},-1\right)$ and is repelling. The point $\left(x(\alpha), m_{0}^{\alpha}(x(\alpha)) \in \Lambda_{0}^{\alpha, u}\right.$ is stable for the induced dynamics.

- If $\alpha>0, \Lambda_{0}^{\alpha}=\Lambda_{0}^{\alpha, s}$ is an increasing curve connecting the points $\left(T_{Y}^{\alpha},-1\right)$ and $\left(T_{X}^{\alpha}, 1\right)$ and is attracting. The point $\left(x(\alpha), m_{0}^{\alpha}(x(\alpha)) \in \Lambda_{0}^{\alpha, s}\right.$ is unstable for the induced dynamics.

- In the invisible-invisible case, see Fig. 13(b)

- If $\alpha<0, \Lambda_{0}^{\alpha}=\Lambda_{0}^{\alpha, s}$ is a decreasing curve connecting the points $\left(T_{X}^{\alpha}, 1\right)$ and $\left(T_{Y}^{\alpha},-1\right)$ and is attracting. The point $\left(x(\alpha), m_{0}^{\alpha}(x(\alpha)) \in \Lambda_{0}^{\alpha, s}\right.$ is stable for the induced dynamics.

- If $\alpha>0, \Lambda_{0}^{\alpha}=\Lambda_{0}^{\alpha, u}$ is an increasing curve connecting the points $\left(T_{Y}^{\alpha},-1\right)$ and $\left(T_{X}^{\alpha}, 1\right)$ and is repelling. The point $\left(x(\alpha), m_{0}^{\alpha}(x(\alpha)) \in \Lambda_{0}^{\alpha, u}\right.$ is unstable for the induced dynamics.

\subsubsection{Folds with opposite visibility}

When the folds have opposite visibility, for $\alpha=0$ there exists a curve $v=m_{0}(x)$ defined in a neighborhood of $x=0$ such that $\mathcal{A}\left(x, m_{0}(x)\right)=0$, where

$$
\begin{aligned}
m_{0}(x) & =\bar{v}+\mathcal{O}(x) \\
\bar{v} & =\varphi^{-1}\left(\frac{\left(X_{x}^{2}+Y_{x}^{2}\right)}{\left(Y_{x}^{2}-X_{x}^{2}\right)}(\mathbf{0})\right)
\end{aligned}
$$

which is transverse to the line $x=0$ at the point $(0, \bar{v})$.

Using (3.23), for $\alpha=0$, we have two hyperbolic critical manifolds

$$
\Lambda_{0}^{s}=\left\{(x, v): v=m_{0}(x), x<0\right\}, \quad \Lambda_{0}^{u}=\left\{(x, v): v=m_{0}(x), x>0\right\}
$$

which are attracting and repelling, respectively. Therefore, $\Lambda_{0}=\Lambda_{0}^{s} \cup \Lambda_{0}^{u}$, see Figures 14(a) and $14(\mathrm{~b})$, for $\alpha=0$. 

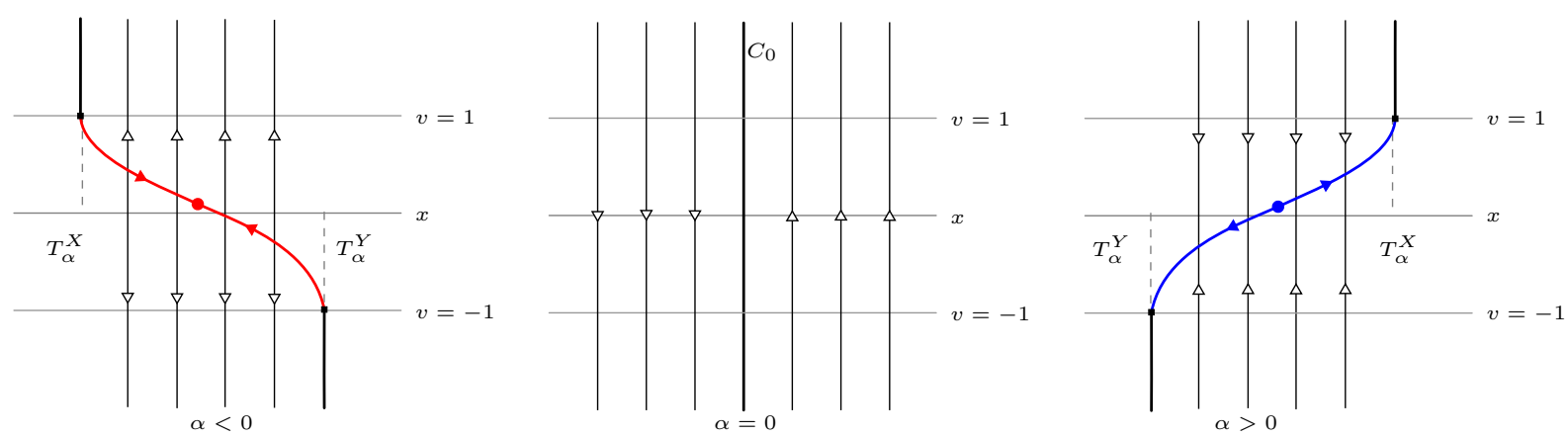

(a) The visible-visible fold
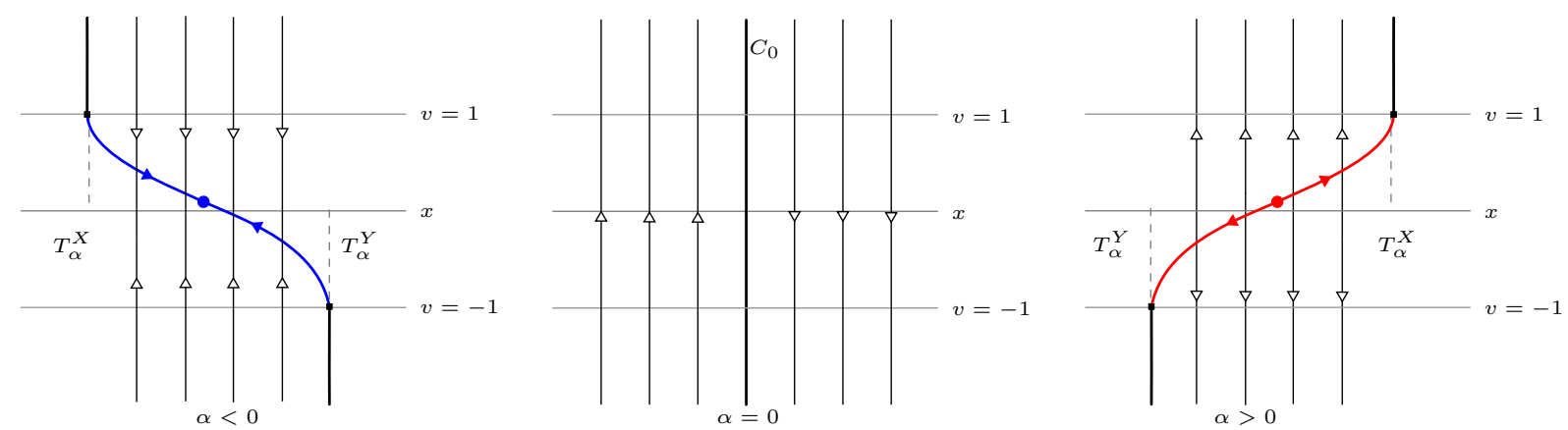

(b) The invisible-invisible fold

Figure 13: The critical manifold when the folds have the same visibility for different values of $\alpha$.

For $\alpha \neq 0$, we have seen in Proposition 2.20 that a crossing region appears between the fold points $\left(T_{X}^{\alpha}, 0\right)$ and $\left(T_{Y}^{\alpha}, 0\right)$. Therefore there exist two critical manifolds: $\Lambda_{0}^{\alpha, s}$, which is defined for $x<\min \left\{T_{X}^{\alpha}, T_{Y}^{\alpha}\right\}$ and is attracting, and $\Lambda_{0}^{\alpha, u}$ which is defined for $x>\max \left\{T_{X}^{\alpha}, T_{Y}^{\alpha}\right\}$ and is repelling.

Adding the results about the sliding and escaping regions of section 2.3 we obtain:

- $(\operatorname{det} Z)_{x}(\mathbf{0})>0$, see Fig. 14(a)

- If $\alpha<0, \Lambda_{0}^{\alpha, s}$ is a increasing and attracting curve containing the point $\left(T_{X}^{\alpha}, 1\right) . \Lambda_{0}^{\alpha, u}$ is a increasing and repelling curve containing the point $\left(T_{Y}^{\alpha},-1\right)$. The point $\left(x(\alpha), m_{0}^{\alpha}(x(\alpha))\right.$ $\in \Lambda_{0}^{\alpha, s}$ is unstable for the induced dynamics.

- If $\alpha>0, \Lambda_{0}^{\alpha, s}$ is a decreasing and attracting curve containing the point $\left(T_{Y}^{\alpha},-1\right) . \Lambda_{0}^{\alpha, u}$ is a decreasing and repelling curve containing the point $\left(T_{X}^{\alpha}, 1\right)$. The point $\left(x(\alpha), m_{0}^{\alpha}(x(\alpha))\right.$ $\in \Lambda_{0}^{\alpha, u}$ is stable for the induced dynamics.

- $(\operatorname{det} Z)_{x}(\mathbf{0})<0$, see Fig. 14(b)

- If $\alpha<0, \Lambda_{0}^{\alpha, s}$ is a increasing and attracting curve containing the point $\left(T_{X}^{\alpha}, 1\right) . \Lambda_{0}^{\alpha, u}$ is a increasing and repelling curve containing the point $\left(T_{Y}^{\alpha},-1\right)$. The point $\left(x(\alpha), m_{0}^{\alpha}(x(\alpha))\right.$ $\in \Lambda_{0}^{\alpha, u}$ is unstable for the induced dynamics.

- If $\alpha>0, \Lambda_{0}^{\alpha, s}$ is a decreasing and attracting curve containing the point $\left(T_{X}^{\alpha}, 1\right) . \Lambda_{0}^{\alpha, u}$ is a decreasing and repelling curve containing the point $\left(T_{Y}^{\alpha},-1\right)$. The point $\left(x(\alpha), m_{0}^{\alpha}(x(\alpha))\right.$ $\in \Lambda_{0}^{\alpha, s}$ is stable for the induced dynamics.

Remark 3.11. When $\alpha$ tends to zero the tangency points $T_{X}^{\alpha}$ and $T_{Y}^{\alpha}$ meet in the fold-fold singularity. Therefore, when the folds have the same visibility, the critical manifold $\Lambda_{0}^{\alpha}$ tends to the vertical line $C_{0}=\{x=0\}$ which is not hyperbolic. When the folds have opposite visibility, the two critical manifolds $\Lambda_{0}^{\alpha, s}$ and $\Lambda_{0}^{\alpha, u}$ join in the degenerated hyperbola $C_{0} \cup \Lambda_{0}^{s} \cup \Lambda_{0}^{u}$ (see Fig. 14(a), Fig. 14(b)). 

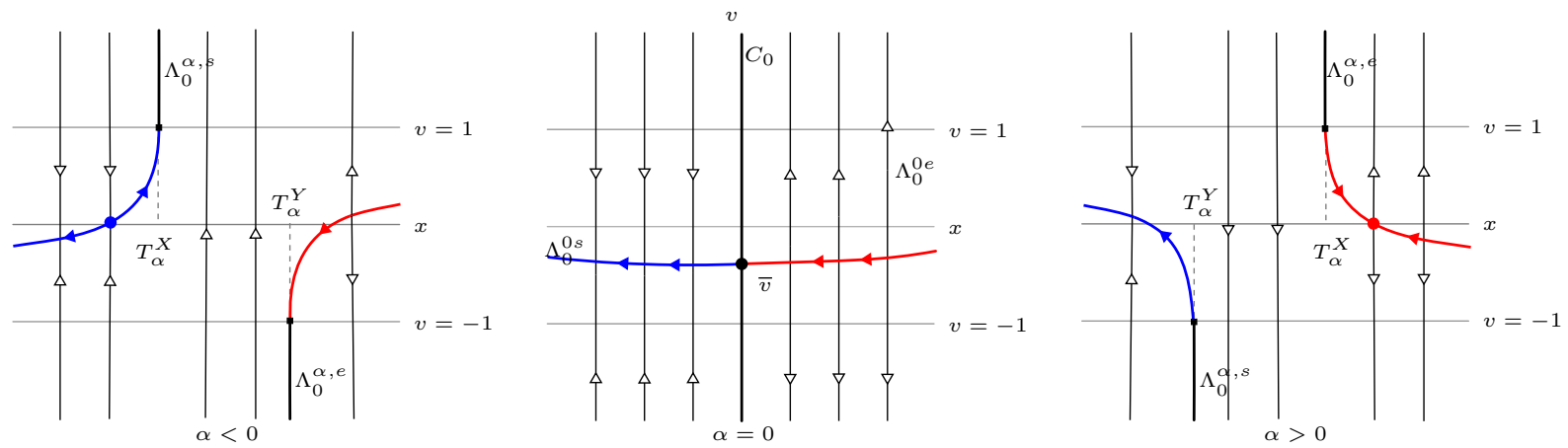

(a) The visible-invisible fold satisfying $(\operatorname{det} Z)_{x}(\mathbf{0})>0$
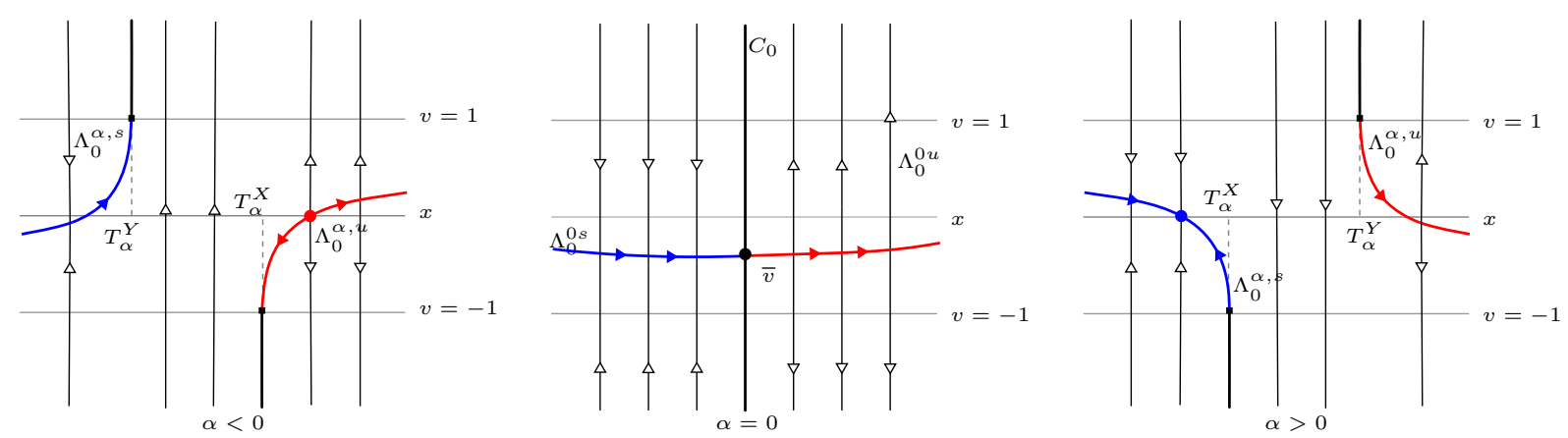

(b) The visible-invisible fold satisfying $(\operatorname{det} Z)_{x}(\mathbf{0})<0$

Figure 14: The critical manifold when the folds have the opposite visibility for different values of $\alpha$.

Remark 3.12. In all cases, the dynamics over the critical manifold for $\alpha \neq 0$ is equivalent to the dynamics of the sliding vector field $\left(Z^{\alpha}\right)^{s}$ studied in section 2. Therefore, for each fixed $\alpha \neq 0$ and $\varepsilon>0$ sufficiently small, the dynamics of the regularized vector field is faithful to the dynamics of the unfoldings of $Z$ studied in that section.

Remark 3.13. The points $v^{*}$, given in (3.12), and $\bar{v}$ given in (3.26) satisfy the following relation

$$
\varphi\left(v^{*}\right)-\varphi(\bar{v})=C(\operatorname{det} Z)_{x}(\mathbf{0}),
$$

where $C=\frac{2}{\left(X^{1}-Y^{1}\right)(\mathbf{0})\left(X_{x}^{2}-Y_{x}^{2}\right)(\mathbf{0})}>0$.

Since $\varphi$ is an increasing map, we have that $-1<\bar{v}<v^{*}<1$, if $(\operatorname{det} Z)_{x}(\mathbf{0})>0$ and $-1<v^{*}<$ $\bar{v}<1$, if $(\operatorname{det} Z)_{x}(\mathbf{0})<0$. The relative positions of the points $\left(0, v^{*}\right)$ and $(0, \bar{v})$ will be important to describe the global dynamics of the regularized vector field in subsection 4.3.

\section{The bifurcation diagram of the regularized vector field $Z_{\varepsilon}^{\alpha}$}

The aim of this section is to understand the relation between the bifurcation diagram of the versal unfolding $Z^{\alpha}$ of $Z \in \Lambda^{F}$ and its regularization $Z_{\varepsilon}^{\alpha}$.

As we will see in section 4.1 the dynamics of the regularized vector field $Z_{\varepsilon}^{\alpha}$ is very similar to the dynamics of the unfolding $Z^{\alpha}$ in the case of the visible-visible fold. When we study the invisible-invisible fold in section 4.2 and the visible-invisible one in section 4.3 we will see that the regularization may create new periodic orbits and bifurcations which were not present in the unfolding $Z^{\alpha}$. 


\subsection{Visible-visible case}

When both folds are visible, by Corolary 3.8 the critical point $P(\alpha, \varepsilon)$ is a saddle which is $\varepsilon$-close to the pseudo-equilibrium $Q(\alpha)$. Using the results in subsubsection 3.2.1 and applying the Fenichel Theorem, for each fixed $\alpha \neq 0$ and any compact set between the fold points $\left(T_{X}^{\alpha}, 1\right)$ and $\left(T_{Y}^{\alpha},-1\right)$, for $0<\varepsilon<\varepsilon_{0}(\alpha)$, there exists a normally hyperbolic invariant manifold $\Lambda_{\varepsilon}^{\alpha}$ which is $\varepsilon$-close to $\Lambda_{0}^{\alpha}$ (see Fig. 13(a) . Moreover, for $\alpha<0, \Lambda_{\varepsilon}^{\alpha}=\Lambda_{\varepsilon}^{\alpha, u}$ is repelling and is the stable manifold of the saddle point $P(\alpha, \varepsilon)$ and for $\alpha>0, \Lambda_{\varepsilon}^{\alpha}=\Lambda_{\varepsilon}^{\alpha, s}$ is attracting and is its the unstable manifold.

A simple computation shows that for each fixed $\alpha \neq 0$ and for $\varepsilon>0$ the vector field $X^{\alpha}(x, \varepsilon v)$ has a unique visible fold point at $v=1$ at $T_{X}^{\alpha, \varepsilon}=T_{X}^{\alpha}+\mathcal{O}(\varepsilon)$ (see (2.18) . Analogously, the vector field $Y^{\alpha}(x, \varepsilon v)$ has a unique visible fold at $v=-1$ at the point $T_{Y}^{\alpha, \varepsilon}=T_{Y}^{\alpha}+\mathcal{O}(\varepsilon)$. Moreover

$$
\begin{aligned}
& T_{X}^{\alpha, \varepsilon}=-\left(\frac{\tilde{X}^{2}}{X_{x}^{2}}(\mathbf{0})\right) \alpha-\left(\frac{X_{y}^{2}}{X_{x}^{2}}(\mathbf{0})\right) \varepsilon+\mathcal{O}_{2}(\alpha, \varepsilon), \\
& T_{Y}^{\alpha, \varepsilon}=-\left(\frac{\tilde{Y}^{2}}{Y_{x}^{2}}(\mathbf{0})\right) \alpha-\left(\frac{Y_{y}^{2}}{Y_{x}^{2}}(\mathbf{0})\right) \varepsilon+\mathcal{O}_{2}(\alpha, \varepsilon) .
\end{aligned}
$$

Observe that for $x<T_{X}^{\alpha, \varepsilon}$ the vector $X^{\alpha}(x, 1)$ points inward to the regularization zone and points outwards to the regularization zone for $x>T_{X}^{\alpha, \varepsilon}$. Analogously, for $x<T_{Y}^{\alpha, \varepsilon}$ the vector $Y^{\alpha}(x,-1)$ points outwards to the regularization zone for $x<T_{Y}^{\alpha, \varepsilon}$ and inwards to the regularization zone for $x>T_{Y}^{\alpha, \varepsilon}$.

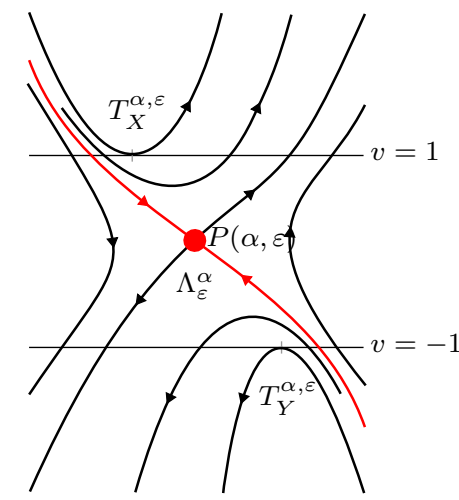

$\alpha<\alpha_{\mathcal{H}}$

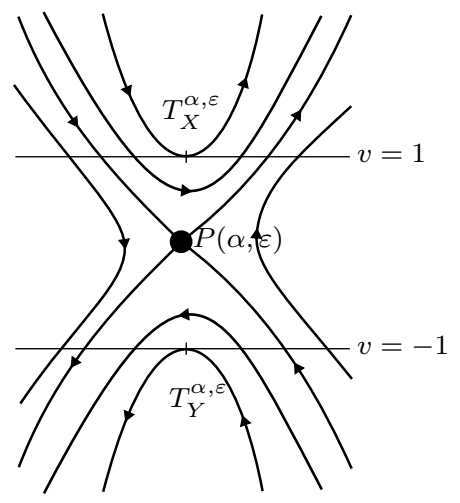

$\alpha=\alpha_{\mathcal{H}}$

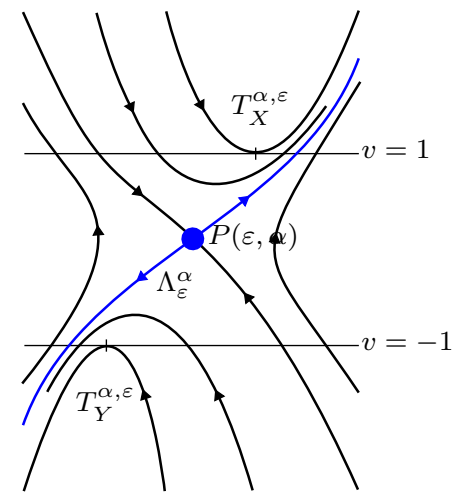

$\alpha>\alpha_{\mathcal{H}}$

Figure 15: The phase portrait of $Z_{\varepsilon}^{\alpha}$.

The above information and the fact that the dynamics over the Fenichel Manifold $\Lambda_{\varepsilon}^{\alpha}$ is equivalent to the one over the critical manifold $\Lambda_{0}^{\alpha}$, gives:

- for $\alpha<0$ and $\varepsilon$ small enough, the Fenichel manifold $\Lambda_{\varepsilon}^{\alpha}$, which is the stable manifold of the saddle point $P(\alpha, \varepsilon)$, intersects the section $\{v=1\}$ on the left of the tangency point $T_{X}^{\alpha, \varepsilon}$ and it intersects the section $\{v=-1\}$ on the right of the tangency point $T_{Y}^{\alpha, \varepsilon}$,

- for $\alpha>0$ and $\varepsilon$ small enough, the Fenichel manifold $\Lambda_{\varepsilon}^{\alpha}$, which is the unstable manifold of the saddle point $P(\alpha, \varepsilon)$, intersects the section $\{v=1\}$ on the right of the tangency point $T_{X}^{\alpha, \varepsilon}$ and it intersects the section $\{v=-1\}$ on the left of the tangency point $T_{Y}^{\alpha, \varepsilon}$.

Observe that, for $\alpha=0$ and $\varepsilon$ small enough, even if one can not apply Fenichel theorem, we know that $P(0, \varepsilon)$ is a saddle with stable and unstable manifolds. By the exposed above, the phase portrait of $Z_{\varepsilon}^{\alpha}$ must look as in Figure 15 .

Remark 4.1. Over the curve $\mathcal{H}$, given in (3.16) the matrix $D Z_{\varepsilon}^{\alpha}(P(\alpha, \varepsilon))$ has two real eigenvalues with same absolute value. Therefore, the critical point $P(\alpha, \varepsilon)$ is a neutral saddle and the qualitative behavior of the system reminds the behavior of the Filippov system $Z^{\alpha}$ for $\alpha=0$. Then in some sense, the dynamics of $Z$ is "continued" over the curve $\mathcal{H}$. 


\subsection{The invisible-invisible case}

When both folds are invisible, for each $\alpha \neq 0$ and $\varepsilon>0$ small enough, by Corolary 3.8 and in agreement with ST98, the point $P(\alpha, \varepsilon)$ is a node with the same character that the pseudo-node $Q(\alpha)$ of $Z^{\alpha}$.

Using the results about the critical manifold given in subsubsection 3.2.1, we can apply the Fenichel Theorem in any compact set between the points $\left(T_{X}^{\alpha}, 1\right)$ and $\left(T_{Y}^{\alpha},-1\right)$, obtaining that for $0<\varepsilon<\varepsilon_{0}(\alpha)$, there exists a normally hyperbolic invariant manifold $\Lambda_{\varepsilon}^{\alpha}$ which is $\varepsilon$-close to $\Lambda_{0}^{\alpha}$ (see Fig. 13(b) . Moreover, for $\alpha<0, \Lambda_{\varepsilon}^{\alpha}=\Lambda_{\varepsilon}^{\alpha, s}$ is attracting and for $\alpha>0, \Lambda_{\varepsilon}^{\alpha}=\Lambda_{\varepsilon}^{\alpha, u}$ is repelling. In both cases, these manifolds contain the node $P(\alpha, \varepsilon)$ and they are its weak manifold.

We now consider the tangency points $T_{X, Y}^{\alpha, \varepsilon}$ given in (4.1) and (4.2), see Figure 16. For $x<$ $T_{X}^{\alpha, \varepsilon}$ the vector $X^{\alpha}(x, 1)$ points outward to the regularization zone and points inwards to the regularization zone for $x>T_{X}^{\alpha, \varepsilon}$. Analogously, for $x<T_{Y}^{\alpha, \varepsilon}$ the vector $Y^{\alpha}(x,-1)$ points inwards to the regularization zone and outwards to the regularization zone for $x>T_{Y}^{\alpha, \varepsilon}$.

The above information and the fact that the dynamics over the Fenichel Manifold $\Lambda_{\varepsilon}^{\alpha}$ is the same of the critical manifold $\Lambda_{0}^{\alpha}$ gives:

- for $\alpha<0$ and $\varepsilon$ small enough, the Fenichel manifold $\Lambda_{\varepsilon}^{\alpha, s}$, which is the weak manifold of the stable node $P(\alpha, \varepsilon)$, intersects the section $\{v=1\}$ on the right of the tangency point $T_{X}^{\alpha, \varepsilon}$ and it intersects the section $\{v=-1\}$ on the left of the tangency point $T_{Y}^{\alpha, \varepsilon}$,

- for $\alpha>0$ and $\varepsilon$ small enough, the Fenichel manifold $\Lambda_{\varepsilon}^{\alpha, u}$, which is the weak manifold of the unstable node $P(\alpha, \varepsilon)$, intersects the section $\{v=1\}$ on the left of the tangency point $T_{X}^{\alpha, \varepsilon}$ and it intersects the section $\{v=-1\}$ on the right of the tangency point $T_{Y}^{\alpha, \varepsilon}$.

The phase portrait of the vector field $Z_{\varepsilon}^{\alpha}$ for $(\alpha, \varepsilon)$ below the parabola $\mathcal{D}$ is given in Figure 16.
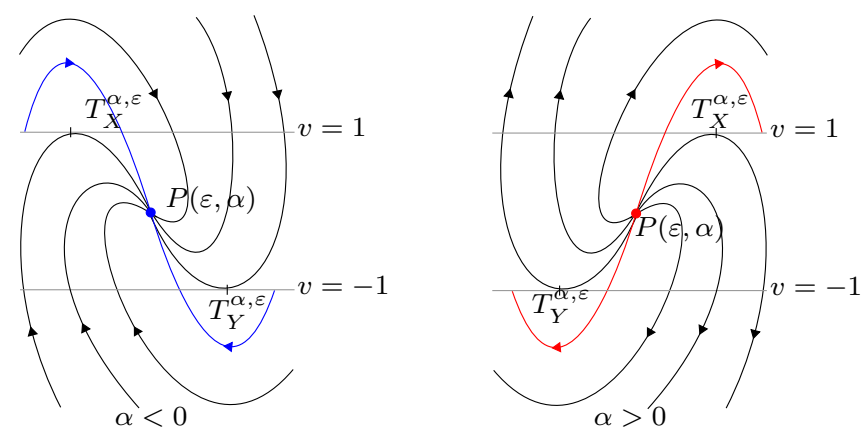

Figure 16: Phase portrait of $Z_{\varepsilon}^{\alpha}$ in the invisible-invisible case for $(\alpha, \varepsilon)$ below the curve $\mathcal{D}$ for $\alpha \neq 0$.

When $(\alpha, \varepsilon)$ are above the parabola $\mathcal{D}$ the point $P(\alpha, \varepsilon)$ is a focus. Since it undergoes a Hopf bifurcation, a periodic orbit arises at one side of the curve $\mathcal{H}$. The nature of the Hopf bifurcation depends on the first Lyapunov coefficient $\ell_{1}(\varepsilon, \alpha(\varepsilon)$ ) (see [HG83, p. 152).

Now we are going to investigate the persistence of the crossing cycle $\Gamma^{\alpha}$ which appears in the unfolding $Z^{\alpha}$ when $\alpha \cdot \mu_{Z}>0$ as seen in Proposition 2.18. We will also study the relation between the limit cycle which raises from the Hopf bifurcation and the periodic orbit $\Gamma_{\varepsilon}^{\alpha, s}$ which raises from the crossing cycle $\Gamma^{\alpha}$.

Proposition 4.2. Consider $Z \in \Lambda^{F}$ having an invisible fold-fold satisfying $X^{1} \cdot Y^{1}(\mathbf{0})<0$. Consider the coefficient $\mu_{Z}$ in (2.15). If $\mu_{Z}>0$, one has that:

(a) For $\alpha, \varepsilon>0$ sufficiently small and such that $(\alpha, \varepsilon)$ are on the right of the curve $\mathcal{H}$ (see (3.16)), there exists a stable periodic orbit $\Gamma_{\varepsilon}^{\alpha, s}$ of the vector field $Z_{\varepsilon}^{\alpha}$;

Moreover,

(b1) If $\alpha>0$, there exists $\varepsilon_{0}(\alpha)$ such that for $0<\varepsilon<\varepsilon(\alpha), \Gamma_{\varepsilon}^{\alpha, s}$ is the unique stable hyperbolic periodic orbit of $Z_{\varepsilon}^{\alpha}$. Furthermore, $\Gamma_{\varepsilon}^{\alpha, s}=\Gamma^{\alpha}+\mathcal{O}(\varepsilon)$, where $\Gamma^{\alpha}$ is given in Proposition 2.18. 
(b2) If $\alpha<0$, the system $Z_{\varepsilon}^{\alpha}$ has no periodic orbits for $0<\varepsilon<\varepsilon(\alpha)$.

(c1) There exists a Melnikov function $M(v, \delta)$ given in (5.3), whose properties are given in Proposition 5.1, such that for $\alpha=\delta \varepsilon+O\left(\varepsilon^{2}\right)$, such that $\delta>\delta_{\mathcal{H}}$ and $\varepsilon$ small enough, calling $\left(v_{\varepsilon}^{s}, 0\right)=\Gamma_{\varepsilon}^{\alpha, s} \cap\{x=0\}=\left(v^{s}, 0\right)+O(\varepsilon)$, the value $v^{s}$ satisfies:

$$
M\left(v^{s}, \delta\right)=0, \frac{\partial M}{\partial v}\left(v^{s}, \delta\right) \leq 0,
$$

and $\Gamma_{\varepsilon}^{\alpha, s}$ is locally unique if $\frac{\partial M}{\partial v}\left(v^{s}, \delta\right)<0$.

(c2) Moreover, if $M(v, \delta)$ is strictly concave $\left(\frac{\partial^{2} M}{\partial v^{2}}(v, \delta)<0\right)$ then the periodic orbit $\Gamma_{\varepsilon}^{\alpha, s}$ is unique and disappears at $\alpha_{\mathcal{H}}$.

If $\mu_{Z}<0$ one has an analogous results changing signs of the parameters.

Proof. The proof of this proposition can be found in subsection 7.1.

Next we analyze the relation between the stable periodic orbit $\Gamma_{\varepsilon}^{\alpha, s}$ and a periodic orbit which arises from the Hopf bifurcation.

The following theorems give us the bifurcation diagram of $Z_{\varepsilon}^{\alpha}$ in each case, depending on the signs of $\mu_{Z}$ and the first Lyapunov coefficient $\ell_{1}(\alpha(\varepsilon), \varepsilon)$.

Theorem 4.3 (Invisible fold-fold: $\mu_{Z}>0$ and $\ell_{1}(\alpha(\varepsilon), \varepsilon)<0$ ). Let $Z \in \Lambda^{F}$ having an invisible fold-fold satisfying $X^{1} \cdot Y^{1}(\mathbf{0})<0$ and $\mu_{Z}>0$. Suppose that the first Lyapunov coefficient $\ell_{1}(\alpha(\varepsilon), \varepsilon)$ at the Hopf bifurcation of $Z_{\varepsilon}^{\alpha}$ is negative. Let $\alpha_{\mathcal{D}}^{ \pm}\left(\varepsilon_{0}\right)$ and $\alpha_{\mathcal{H}}\left(\varepsilon_{0}\right)$ be the intersections between the line $\varepsilon=\varepsilon_{0}$ and the curves $\mathcal{D}^{ \pm}$(the negative and positive parts of $\mathcal{D}$ given in (3.15) and $\mathcal{H}((3.16)$, respectively. One has that

- For $\alpha<\alpha_{\mathcal{D}}^{-}\left(\varepsilon_{0}\right)$ sufficiently small the critical point $P(\alpha, \varepsilon)$ is an stable node;

- For $\alpha_{\mathcal{D}}^{-}\left(\varepsilon_{0}\right)<\alpha<\alpha_{\mathcal{H}}\left(\varepsilon_{0}\right)$ the critical point $P(\alpha, \varepsilon)$ is an stable focus;

- When $\alpha=\alpha_{\mathcal{H}}\left(\varepsilon_{0}\right)$ a supercritical Hopf bifurcation takes place;

- For $\alpha$ values such that $\alpha_{\mathcal{H}}\left(\varepsilon_{0}\right)<\alpha<\alpha_{\mathcal{D}}^{+}\left(\varepsilon_{0}\right)$, the critical point $P(\alpha, \varepsilon)$ is an unstable focus and there exist a stable limit cycle $\Gamma_{\varepsilon}^{\alpha, s}$.

- When $\alpha>\alpha_{\mathcal{D}}^{+}\left(\varepsilon_{0}\right)$ the critical point $P(\alpha, \varepsilon)$ is a unstable node and the limit cycle $\Gamma_{\varepsilon}^{\alpha, s}$ persists for $\alpha>\alpha_{\mathcal{D}}^{+}\left(\varepsilon_{0}\right)$. The cycle $\Gamma_{\varepsilon}^{\alpha, s}$ tends to $\Gamma^{\alpha}$ when $\varepsilon$ goes to zero.

- If the Melnikov function $M(v, \delta)$ is strictly concave for $\delta$ close enough to $\delta_{\mathcal{H}}$ the stable periodic $\Gamma_{\varepsilon}^{\alpha, s}$ disappears at the Hopf bifurcation. Therefore, the periodic orbit which rises from the Hopf bifurcation and the one which is given by Proposition 4.2 are the same.

Proof. The character of the critical point $P(\alpha, \varepsilon)$ is given by Corolary 3.8. The existence of the periodic orbit $\Gamma_{\varepsilon}^{\alpha, s}$ is given by Proposition 4.2. Moreover, when the first Lyapunov coefficient $\ell_{1}(\varepsilon, \alpha(\varepsilon))$ is negative, a supercritical Hopf bifurcation occurs, creating a stable periodic orbit $\tilde{\Gamma}_{\varepsilon}^{\alpha, s}$ near the critical point $P(\alpha, \varepsilon)$ for $(\alpha, \varepsilon)$ to the right of the curve $\mathcal{H}$. As both periodic orbits are given, in first order, by the zeros of $M(v, \delta)$, when this function is strictly concave both periodic orbits have to coincide.

The cycle $\Gamma_{\varepsilon}^{\alpha, s}$, given by Theorem 4.3 , tends to the non smooth crossing cycle $\Gamma^{\alpha}$ when $\varepsilon$ tends to zero. Summarizing, the small orbit arising from the Hopf bifurcation and the regularized periodic orbit coming from the pseudo-Hopf bifurcation of the non smooth system are, generically, continuation one of the other.

The bifurcation diagram of $Z_{\varepsilon}^{\alpha}$ in the two parameter space is sketched in Figure 17 


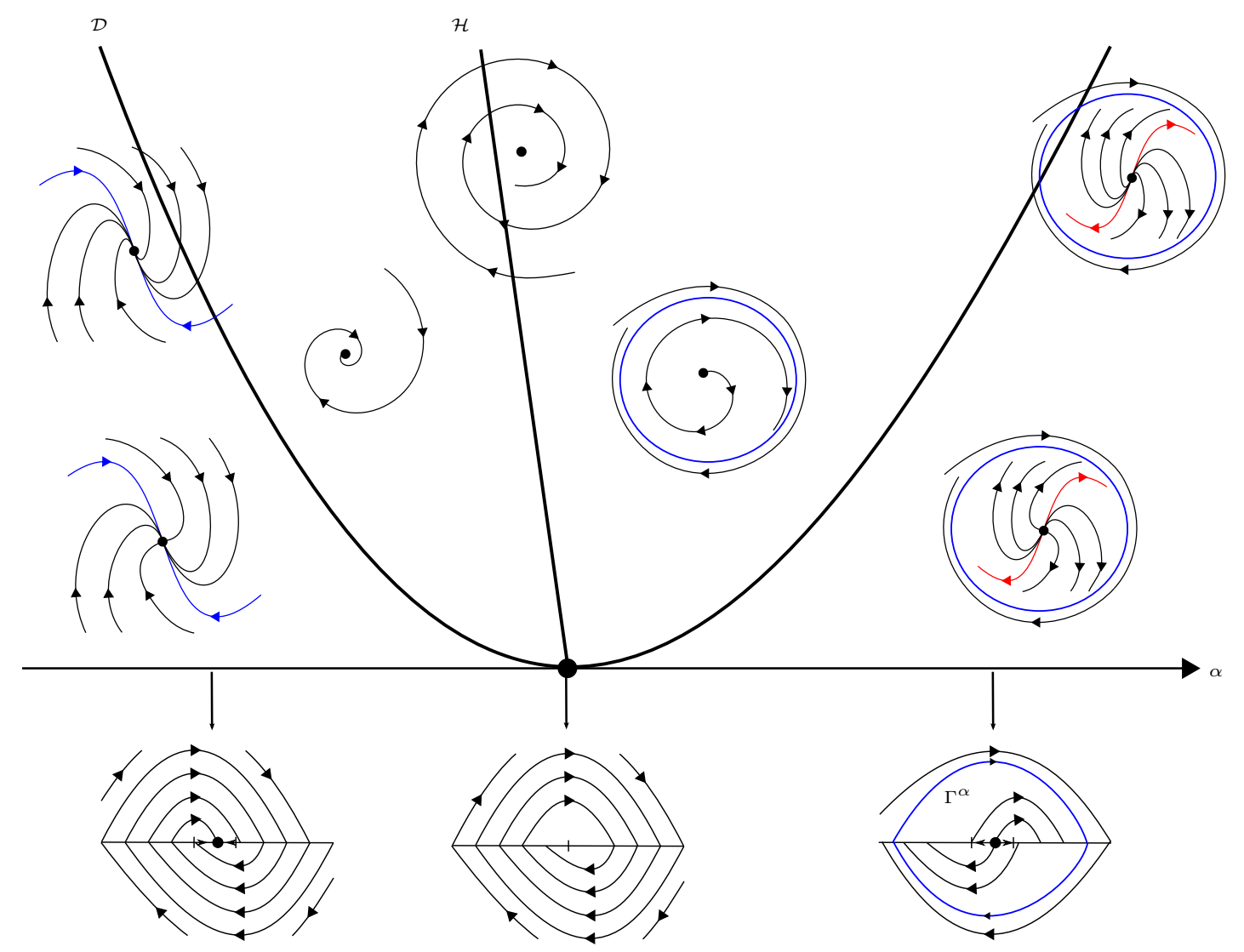

Figure 17: Bifurcation diagram of $Z_{\varepsilon}^{\alpha}$ when $\ell_{1}(\varepsilon, \alpha(\varepsilon))<0$ and $Z$ has an invisible fold-fold with $\mu_{Z}>0$. A stable limit cycle exists for $\alpha>\alpha_{\mathcal{H}}$.

Theorem $4.4\left(\mu_{Z}>0\right.$ and $\left.\ell_{1}(\alpha(\varepsilon), \varepsilon)>0\right)$. Assume the same hypothesis of Theorem 4.3 but $\ell_{1}(\alpha(\varepsilon), \varepsilon)$ positive. Then one has that:

- For $\alpha<\alpha_{\mathcal{D}}^{-}\left(\varepsilon_{0}\right)$ sufficiently small the critical point $P(\alpha, \varepsilon)$ is a stable node;

- For $\alpha_{\mathcal{D}}^{-}\left(\varepsilon_{0}\right)<\alpha<\alpha_{\mathcal{H}}\left(\varepsilon_{0}\right)$ the critical point $P(\alpha, \varepsilon)$ is a stable focus;

- There exists a curve $\mathcal{S}$ in the parameter plane such that for $\alpha<\alpha_{\mathcal{S}}\left(\varepsilon_{0}\right)$ the generalized Poincaré return map $\phi_{\varepsilon}^{\alpha}$ has no fixed points.

- For $\alpha_{\mathcal{S}}\left(\varepsilon_{0}\right)<\alpha<\alpha_{\mathcal{H}}\left(\varepsilon_{0}\right)$, the critical point $P(\alpha, \varepsilon)$, which is a stable focus, coexists with a pair of periodic orbits $\Gamma_{\varepsilon}^{\alpha, s}$ and $\Delta_{\varepsilon}^{\alpha, u}$, which are stable and unstable, respectively. For $\alpha=\alpha_{\mathcal{H}}\left(\varepsilon_{0}\right)$ a subcritical Hopf bifurcation takes place. This implies the disappearance of the unstable periodic orbit $\Delta_{\varepsilon}^{\alpha, u}$ for $\alpha>\alpha_{\mathcal{H}}$;

- For $\alpha>\alpha_{\mathcal{H}}\left(\varepsilon_{0}\right)$, the stable limit cycle $\Gamma_{\varepsilon}^{\alpha, s}$ persists. Moreover, the critical point $P(\alpha, \varepsilon)$ is a unstable focus $\alpha_{\mathcal{H}}\left(\varepsilon_{0}\right)<\alpha<\alpha_{\mathcal{D}}^{+}(\varepsilon 0)$ and becomes a unstable node when $\alpha>\alpha_{\mathcal{D}}^{+}\left(\varepsilon_{0}\right)$. The cycle $\Gamma_{\varepsilon}^{\alpha, s}$ tends to $\Gamma^{\alpha}$ when $\varepsilon$ goes to zero.

Proof. The character of the critical point $P(\alpha, \varepsilon)$ is given by Corolary 3.8. The existence of the stable periodic orbit $\Gamma_{\varepsilon}^{\alpha, s}$ for $\alpha>\alpha_{\mathcal{H}}\left(\varepsilon_{0}\right)$, is given by Proposition 4.2. When the parameter values reach the curve $\mathcal{H}$, a subcritical Hopf bifurcation occurs and an unstable periodic orbit $\Delta_{\varepsilon}^{\alpha, u}$ appears for $\alpha<\alpha_{\mathcal{H}}\left(\varepsilon_{0}\right)$. Observe that, for these parameter values, because of the attracting character of the generalized first return map $\phi_{\varepsilon}^{\alpha}$ far from the origin, and the presence of the unstable periodic orbit $\Delta_{\varepsilon}^{\alpha, u}$, the Poincaré-Bendixson theorem guarantees the persistence of the periodic orbit $\Gamma_{\varepsilon}^{\alpha, s}$ for $\alpha<\alpha_{\mathcal{H}}\left(\varepsilon_{0}\right)$ small enough. On the other hand, if we fix $\alpha<0$, Proposition 4.2 says that system 
$Z_{\varepsilon}^{\alpha}$ has no periodic orbit for $\varepsilon<\varepsilon(\alpha)$. Therefore, must exist a curve $\mathcal{S}$ in the parameter space where the "total" first return map $\phi_{\varepsilon}^{\alpha}$ has a bifurcation. Therefore there exists a value $\alpha_{\mathcal{S}}\left(\varepsilon_{0}\right)$, where these two periodic orbits collide for $\alpha=\alpha_{\mathcal{S}}\left(\varepsilon_{0}\right)$ and then disappear for $\alpha<\alpha_{\mathcal{S}}\left(\varepsilon_{0}\right)$.

The next proposition, whose proof is given in section 5 , provides quantitative information about the periodic orbits given in theorem 4.4 in terms of the Melnikov function $M(v, \delta)$,.

Proposition 4.5 (The Saddle-node bifurcation). Consider the Melnikov function $M(v, \delta)$ given in (5.3). Then, under the hypotheses of Theorem 4.4:

- For $\alpha=\delta \varepsilon+O\left(\varepsilon^{2}\right)$, such that $\delta<\delta_{\mathcal{H}}$ sufficiently close, and $\varepsilon$ small enough, calling $\left(v_{\varepsilon}^{u}, 0\right)=$ $\Delta_{\varepsilon}^{\alpha, u} \cap\{x=0\}=\left(v^{u}, 0\right)+O(\varepsilon)$, the value $v^{u}$ satisfies:

$$
M\left(v^{u}, \delta\right)=0, \frac{\partial M}{\partial v}\left(v^{u}, \delta\right)>0,
$$

- If moreover we assume that $\frac{\partial^{3} M}{\partial v^{3}}(v, \delta) \neq 0$ for $\delta$ near $\delta_{\mathcal{H}}$, for $\delta_{\mathcal{S}}<\delta<\delta_{\mathcal{H}}$, the function $M$ has two zeros corresponding to the periodic orbits $\Delta_{\varepsilon}^{\alpha, u}$ and $\Gamma_{\varepsilon}^{\alpha, s}$ given in Theorem 4.4. When $\alpha=\alpha_{\mathcal{S}}\left(\varepsilon_{0}\right)$ a Saddle-Node bifurcation takes place.

- The curve $\mathcal{S}$ can be found as $\alpha=\alpha_{\mathcal{S}}=\delta_{\mathcal{S}} \varepsilon+O\left(\varepsilon^{2}\right)$, where $\delta_{\mathcal{S}}$ is the solution of the (linear in $\delta)$ equations:

$$
M\left(v_{\mathcal{S}}, \delta_{\mathcal{S}}\right)=0, \frac{\partial M}{\partial v}\left(v_{\mathcal{S}}, \delta_{\mathcal{S}}\right)=0
$$

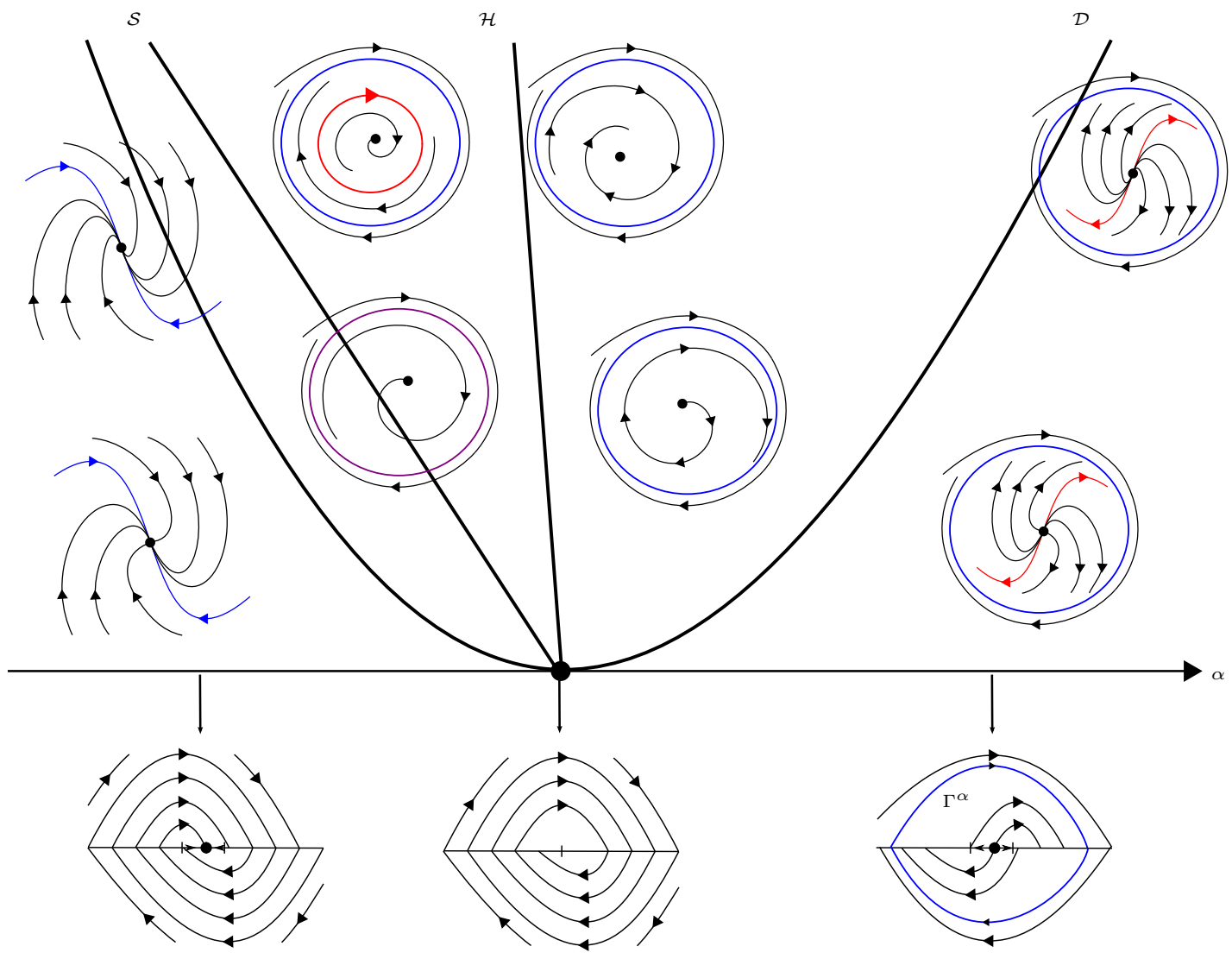

Figure 18: Bifurcation diagram of $Z_{\varepsilon}^{\alpha}$ when $\ell_{1}(\varepsilon, \alpha(\varepsilon))>0$ and $Z$ has an invisible-invisible fold with $\mu_{Z}>0$. Two periodic orbits arise at a saddle-node bifurcation, the unstable one disappears at the Hopf bifurcation. 
In the next two examples, which satisfy Equation (2.8) and therefore are versal unfoldings of the fold-fold singularity, we illustrate the behaviors stated in Theorems 4.3 and 4.4 . We also show that both behaviors can be achieved by the same piecewise vector field considering different transition maps $\varphi$. For this purpose, let $Z^{\alpha}=(X, Y)$ be the piecewise vector field given by

$$
Z^{\alpha}(x, y)=\left\{\begin{array}{l}
X^{\alpha}(x, y)=(1-2 x,-x+\alpha) \\
Y(x, y)=(-1,-x)
\end{array}\right.
$$

which has an invisible-invisible fold at the origin for $\alpha=0$. The coefficient $\mu_{Z}=\frac{4}{3}$, therefore, the origin is an stable fixed point for the Poincaré map $\phi_{Z}$.

The $\varphi$-regularization $Z_{\varepsilon}^{\alpha}(x, y)$ in coordinates $y=\varepsilon v$, is given by

$$
\left\{\begin{array}{l}
\dot{x}=-2 x+2 \varphi(v)(1-x) \\
\varepsilon \dot{v}=-2 x+\alpha(1+\varphi(v))
\end{array} \quad|v| \leq 1 .\right.
$$

Example 4.1 (Supercritical Hopf bifurcation for the invisible-invisible fold). Consider $\varphi$ as in (3.2) with

$$
\varphi(v)=-\frac{1}{2} v^{3}+\frac{3}{2} v, \text { for } v \in(-1,1) .
$$

The critical point is $P(\alpha, \varepsilon)=\left(\frac{1}{2} \alpha, 0\right)+\mathcal{O}_{2}(\alpha, \varepsilon)$, and the curves $\mathcal{D}$ and $\mathcal{H}$ are given by:

$$
\begin{aligned}
\mathcal{D} & =\left\{(\alpha, \varepsilon): \varepsilon=\frac{3}{32} \alpha^{2}+\mathcal{O}\left(\alpha^{3}\right)\right\} \\
\mathcal{H} & =\left\{(\alpha, \varepsilon): \alpha=\frac{4}{3} \varepsilon+\mathcal{O}\left(\varepsilon^{2}\right)\right\}
\end{aligned}
$$

The first Lyapunov coefficient is $\ell_{1}(\alpha(\varepsilon), \varepsilon)=\frac{1}{\sqrt{\varepsilon}}\left(-\frac{1}{3 \sqrt{6}}+\mathcal{O}(\varepsilon)\right)$, therefore the Hopf bifurcation is supercritical.

We fix $\varepsilon=0.006$ and vary the parameter $\alpha$. The intersection of the line $\varepsilon=0.006$ with the parabola $\mathcal{D}$ occurs at the points $\alpha_{\mathcal{D}}^{ \pm} \approx \pm 0.25$ and the intersection with $\mathcal{H}$ at $\alpha_{\mathcal{H}} \approx 0.008$.

In Figure 19 we can see the changes on the phase portrait of $Z_{\varepsilon}^{\alpha}$ when we vary the parameter $\alpha$. In Fig. 19(b) $\alpha>\alpha_{\mathcal{D}}^{-}$: the node becomes a stable focus. In Fig. 19(c) $\alpha_{\mathcal{D}}^{-}<\alpha<\alpha_{\mathcal{H}}$ : the stable focus begins to loose strength. In Fig. 19(d), $\alpha>\alpha_{\mathcal{H}}$ : the critical point is an unstable focus and a small stable limit cycle $\Gamma_{\varepsilon}^{\alpha, s}$ inside the regularization zone appears. In Fig. 19(e) the stable limit cycle $\Gamma_{\varepsilon}^{\alpha, s}$ is no more located inside the regularization zone. In Fig. 19(f) $\alpha>\alpha_{\mathcal{D}}^{+}$: the critical point becomes an unstable node and the limit cycle still $\Gamma_{\varepsilon}^{\alpha, s}$ persists outside the regularization zone. In Fig. 21(a) we show the behavior of the Melnikov function $M(v ; \delta)$, which is strictly concave, for different values of $\delta$ and which has a zero for $\delta>\delta_{\mathcal{H}}$ corresponding to $\Gamma_{\varepsilon}^{\alpha, s}$.

Example 4.2 (Subcritical Hopf bifurcation for the invisible-invisible fold). Consider the transition map

$$
\varphi(v)=-v^{5}+\frac{3}{2} v^{3}+\frac{v}{2} \text { for } v \in(-1,1) .
$$

The critical is point $P(\alpha, \varepsilon)=\left(\frac{1}{2} \alpha, 0\right)+\mathcal{O}_{2}(\alpha, \varepsilon)$, and the bifurcation curves are given by

$$
\begin{aligned}
\mathcal{D} & =\left\{(\alpha, \varepsilon): \varepsilon=\frac{1}{32} \alpha^{2}+\mathcal{O}\left(\alpha^{3}\right)\right\} \\
\mathcal{H} & =\left\{(\alpha, \varepsilon): \alpha=4 \varepsilon+\mathcal{O}\left(\varepsilon^{2}\right)\right\}
\end{aligned}
$$

The Lyapunov coefficient is $\ell_{1}(\varepsilon, \alpha(\varepsilon))=\frac{1}{\sqrt{\varepsilon}}\left(\frac{9}{\sqrt{2}}+\mathcal{O}(\varepsilon)\right)$, therefore the Hopf bifurcation is subcritical.

Fix $\varepsilon=0.006$. In Figure 20, we present the simulations for different values of $\alpha$. In Fig. 20(a), $\alpha=0$ : the stable focus $P(\alpha, \varepsilon)$ is a global attractor. In Figure 20(b), $\alpha=0.012$ : we detect the 


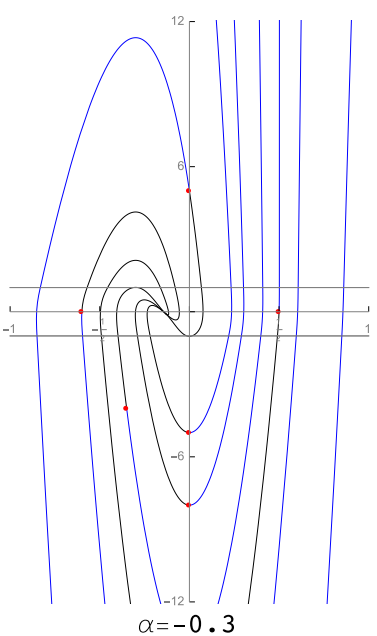

(a)

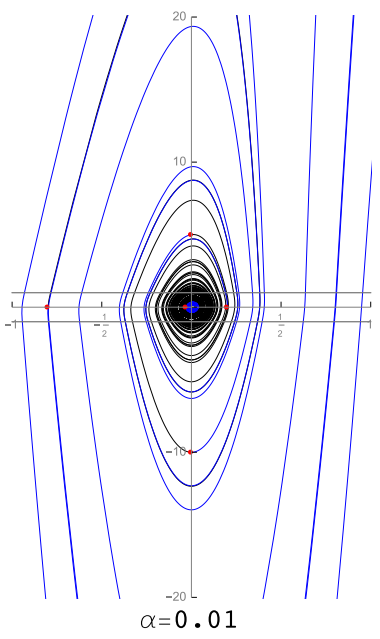

(d)

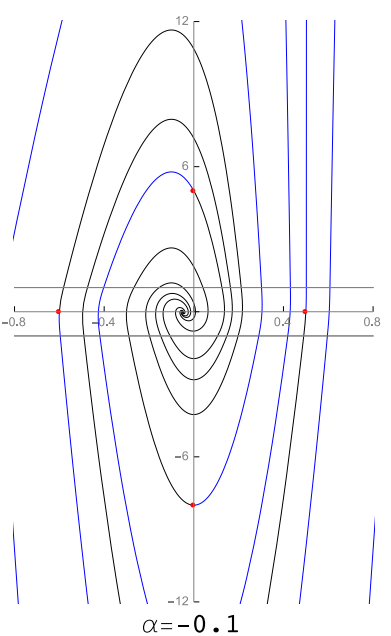

(b)

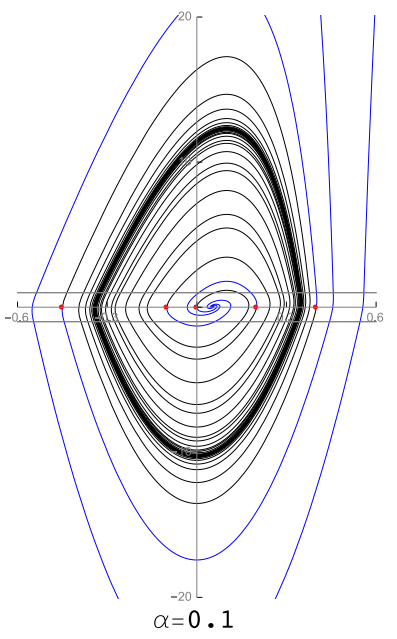

(e)

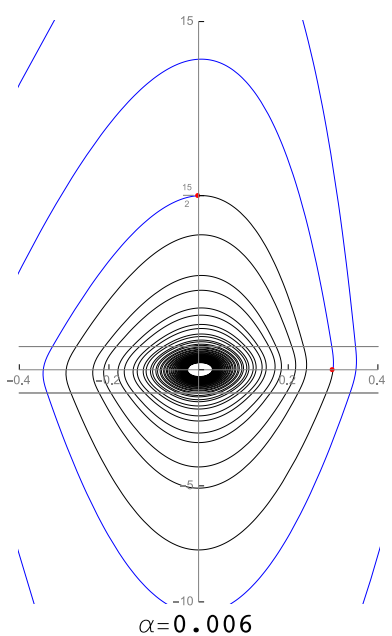

(c)

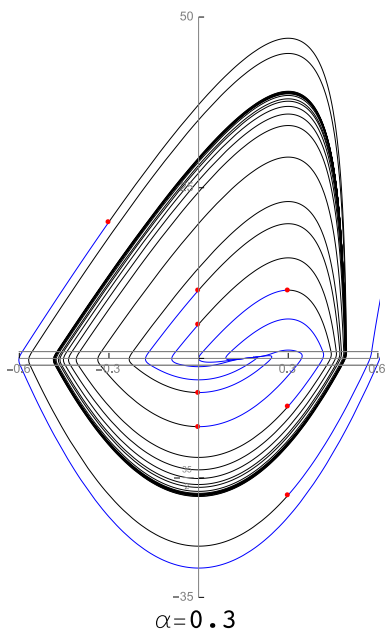

(f)

Initial condition

Negative time

Positive Time

Figure 19: Example 4.1- The evolution of the dynamics while increasing the value of $\alpha$ for $\varepsilon=0.006$.

presence of two periodic orbits, an smaller one $\Delta_{\varepsilon}^{\alpha, u}$ which is unstable and a bigger one $\Gamma_{\varepsilon}^{\alpha, s}$ which is stable. This means that the saddle-node value $\alpha_{\mathcal{S}}$ belongs to the interval $I_{\mathcal{S}}=(0.011,0.012)$. In Figures 20(b), 20(c) and 20(d), we can see that the amplitude of the stable periodic orbit $\Gamma_{\varepsilon}^{\alpha, s}$ increases whereas the unstable one $\Delta_{\varepsilon}^{\alpha, u}$ decreases while we increase the value of $\alpha$. In Fig. 20(e) and Fig. 20(f), for $\alpha$ greater than $\alpha \mathcal{H}$, the critical point $P(\alpha, \varepsilon)$ is an unstable focus and only the stable periodic orbit $\Gamma_{\varepsilon}^{\alpha, s}$ persists. We also show, in Fig. 21(b) the behavior of the Melnikov function $M(v ; \delta)$ for different values of $\delta$. In yellow we show $M\left(v, \delta_{\mathcal{S}}\right)$ which has a zero and is also a maximum. For $\delta_{\mathcal{S}}<\delta<\delta_{\mathcal{H}}$ the function has two zeros. At $\delta=\delta_{\mathcal{H}}$, in black, the zero with positive slope disappears and only the big one with negative slope persists for $\delta>\delta_{\mathcal{H}}$, corresponding to $\Gamma_{\varepsilon}^{\alpha, s}$.

\subsection{The visible-invisible case}

In this section we study the case where the folds have opposite visibility, the vector field $Z$ satisfies $X^{1} \cdot Y^{1}(\mathbf{0})<0$ and $(\operatorname{det} Z)_{x}(\mathbf{0}) \neq 0$, (see (2.9)). The dynamics of the system $Z_{\varepsilon}^{\alpha}$ is more involved when $(\operatorname{det} Z)_{x}(\mathbf{0})<0$, and it is studied in next subsection. 


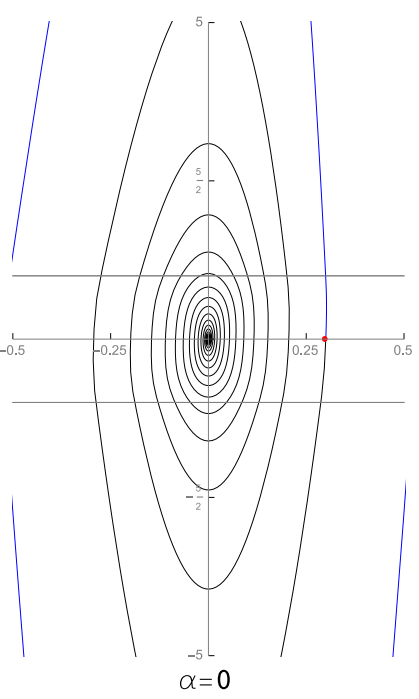

(a)

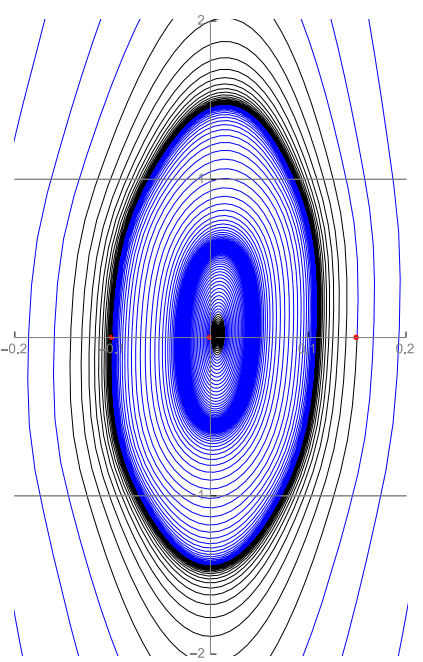

$\alpha=0.015$

(d)

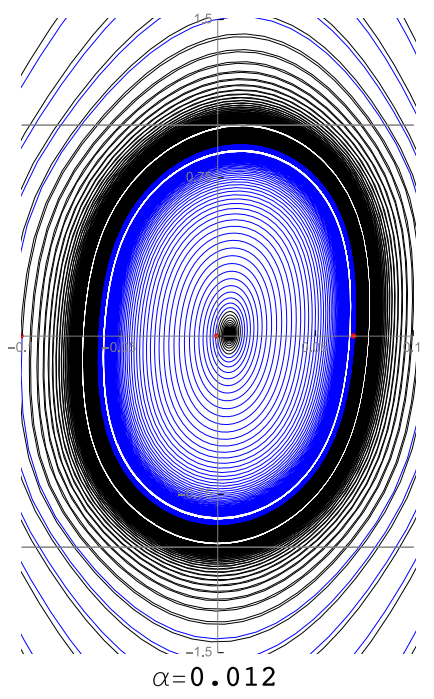

(b)

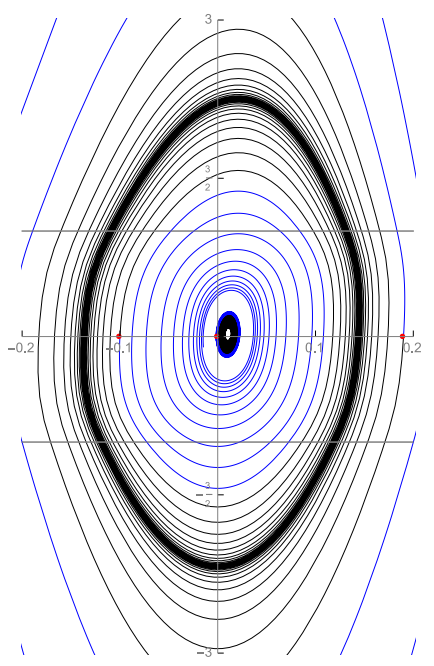

$\alpha=0.021$

(e)

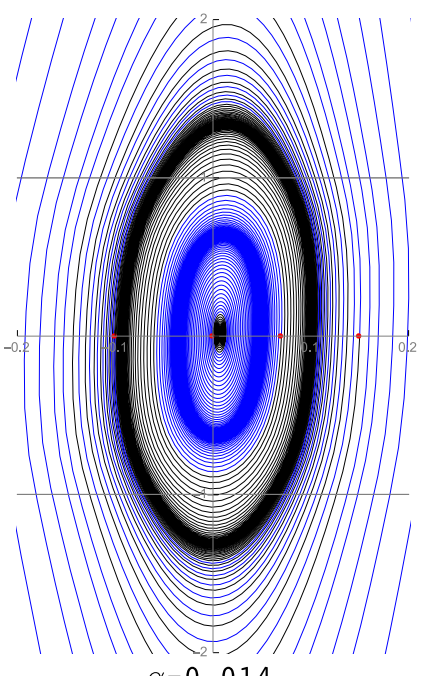

$\alpha=0.014$

(c)

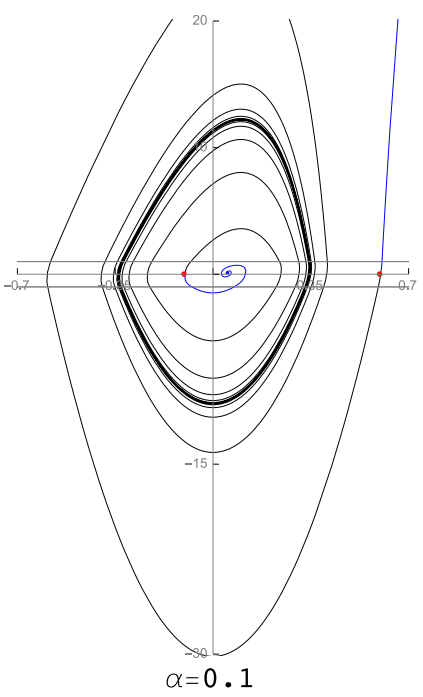

(f)

Initial condition

Negative time

Positive Time

Figure 20: Example 4.2. The evolution of the dynamics while increasing the value of $\alpha$ for $\varepsilon=0.006$.

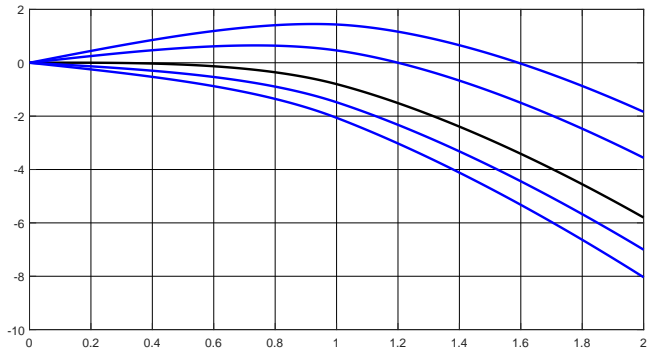

(a)

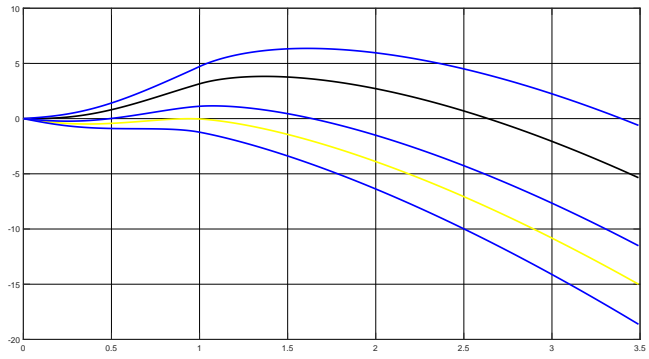

(b)

Figure 21: (a) The Melnikov function for example 4.1 $\delta=\delta_{\mathcal{H}}$ in black is supercritical, $M(v, \delta)$ has a zero with negative slope for $\delta<\delta_{\mathcal{H}}$. (b) The Melnikov function for example 4.2 . $\delta=\delta_{\mathcal{H}}$ in black is subcritical, $\delta=\delta_{S}$ in yellow. For $\delta_{S}<\delta<\delta_{\mathcal{H}} M$ has two zeros, only one survives for $\delta_{\mathcal{H}}<\delta$. 


\subsubsection{The focus case: $(\operatorname{det} Z)_{x}(0)<0$}

When $(\operatorname{det} Z)_{x}(\mathbf{0})<0$, for each $\alpha \neq 0$ and $\varepsilon$ small enough, by Corolary 3.8 the critical point $P(\alpha, \varepsilon)$ is a node with the same character that the pseudo-node $Q(\alpha)$ of $Z^{\alpha}$. This behavior persists for all values of $(\alpha, \varepsilon)$ below the parabola $\mathcal{D}$ given in $(3.15)$.

Using the results about the critical manifolds given in subsubsection 3.2.2 and applying Fenichel theory, for each fixed $\alpha \neq 0$ and any compact set of the critical manifolds $\Lambda_{0}^{\alpha, s}$ and $\Lambda_{0}^{\alpha, u}$ excluding the tangency points $\left(T_{X}^{\alpha}, 1\right)$ and $\left(T_{Y}^{\alpha},-1\right)$, for $0<\varepsilon<\varepsilon_{0}(\alpha)$, there exist two normally hyperbolic invariant manifold $\Lambda_{\varepsilon}^{\alpha, s}$ and $\Lambda_{\varepsilon}^{\alpha, u}$ which are $\varepsilon$-close to $\Lambda_{0}^{\alpha, s}$ and $\Lambda_{0}^{\alpha, u}$, respectively (see Fig. 14(b)).

Moreover, in this case, the Fenichel manifold $\Lambda_{\varepsilon}^{\alpha, u}$ is the weak manifold of the unstable node $P(\alpha, \varepsilon)$ for $\alpha<0$ and $\Lambda_{\varepsilon}^{\alpha, s}$ is the weak manifold of the stable node $P(\alpha, \varepsilon)$ for $\alpha>0$.

As we have seen in subsection 4.1. the vector field $X^{\alpha}(x, \varepsilon v)$ has a unique visible fold point at $\left(T_{X}^{\alpha, \varepsilon}, 1\right)$ and $Y^{\alpha}(x, \varepsilon v)$ has a unique invisible fold point at $\left(T_{Y}^{\alpha, \varepsilon},-1\right)$ (see Equation (4.1) and Equation (4.2).

Observe that for $x<T_{X}^{\alpha, \varepsilon}$ the vector $X^{\alpha}(x, 1)$ points inward to the regularization zone and points outwards to the regularization zone for $x>T_{X}^{\alpha, \varepsilon}$. Analogously, for $x<T_{Y}^{\alpha, \varepsilon}$ the vector $Y^{\alpha}(x,-1)$ points inwards to the regularization zone for $x<T_{Y}^{\alpha, \varepsilon}$ and outwards to the regularization zone for $x>T_{Y}^{\alpha, \varepsilon}$.

The above information and the fact that the dynamics over the Fenichel Manifolds $\Lambda_{\varepsilon}^{\alpha, s / u}$ is equivalent to the one over the critical manifolds $\Lambda_{0}^{\alpha, s / u}$, it follows that (see Figure 22):

- for $\alpha<0$, the stable Fenichel manifold $\Lambda_{\varepsilon}^{\alpha, s}$ intersects the section $\{v=1\}$ on the right of the tangency point $T_{X}^{\alpha, \varepsilon}$. The unstable Fenichel manifold $\Lambda_{\varepsilon}^{\alpha, u}$, which is the weak manifold of the unstable node $P(\alpha, \varepsilon)$, can intersect or not the section $v=-1$. If this intersection occurs it is located to the right of the tangency point $T_{Y}^{\alpha, \varepsilon}$,

- for $\alpha>0$, the unstable Fenichel manifold $\Lambda_{\varepsilon}^{\alpha, u}$ intersects the section $\{v=1\}$ on the left of the tangency point $T_{X}^{\alpha, \varepsilon}$. The stable Fenichel manifold $\Lambda_{\varepsilon}^{\alpha, s}$, which is the weak manifold of the stable node, can intersect or not the section $v=-1$. If this intersection occurs it is located to the left of the tangency point $T_{Y}^{\alpha, \varepsilon}$.

When $(\alpha, \varepsilon)$ is above the parabola $\mathcal{D}$ the critical point $P(\alpha, \varepsilon)$ becomes a focus, which is unstable for $\alpha<\alpha_{\mathcal{H}}(\varepsilon)$, undergoes a Hopf bifurcation for $\alpha=\alpha_{\mathcal{H}}(\varepsilon)$ and is stable for $\alpha>\alpha_{\mathcal{H}}(\varepsilon)$. The main point here is that, since there are no periodic orbits in the bifurcation diagram of $Z^{\alpha}, \alpha \neq 0$, it must exist a curve in the parameter space such that, on this curve, the limit cycle which raises from the Hopf bifurcation disappears. It is at this point that the slow-fast nature of system (3.5) plays a role, because the evolution of the periodic orbit will be influenced by the evolution of the Fenichel manifolds when the parameters vary.

When $\alpha=\delta \varepsilon$, the critical manifolds of $Z_{\varepsilon}^{\alpha}$ are the same as the ones for $\alpha=0$. Then, as we saw in subsubsection 3.2.2 there are two critical manifolds $\Lambda_{0}^{s, u}$ given by:

$$
\Lambda_{0}^{s}=\left\{(x, v), v=m_{0}(x), x<0\right\}, \Lambda_{0}^{u}=\left\{(x, v), v=m_{0}(x), x>0\right\},
$$

which are normally hyperbolic (attracting and repelling respectively) and we restrict them to $|x|>$ $\kappa$ for a small but fixed $\kappa>0$. Applying Fenichel theorem, for any compact subset $\mathcal{K}$ of the critical manifolds, and $\varepsilon$ small enough, we know the existence of two normally hyperbolic invariant manifolds

$$
\Lambda_{\varepsilon}^{s}=\left\{(x, v) \in \mathcal{K}, v=m^{s}(x ; \varepsilon), x<-\kappa\right\}, \Lambda_{\varepsilon}^{u}=\left\{(x, v) \in \mathcal{K}, v=m^{u}(x ; \varepsilon), x>\kappa\right\},
$$

with $m^{s, u}(x ; \varepsilon)=m_{0}(x)+\mathcal{O}(\varepsilon)$.

In Proposition 4.6 we prove the existence of a maximal Canard by looking for $\alpha=\delta_{\mathcal{C}} \varepsilon+\mathcal{O}\left(\varepsilon^{\frac{3}{2}}\right)$ such that the stable and unstable Fenichel manifolds can be extended up to $x=0$ and coincide.

Moreover, we will see in Theorem 4.10 that when $\varphi$ is linear, the regularized system $Z_{\varepsilon}^{\alpha}$ in the regularization zone $|v| \leq 1$ can be transformed, after a change of variables, into a normal form 
studied by Krupa-Szmolyan in KS01b. This will completely determine the position of the curves where the Hopf bifurcation and the maximal Canard occur. Therefore, in this case, we provide a complete description of the bifurcation diagram of the regularized system. Later, in Example 4.4. we see that this result is not true when $\varphi$ is not a linear map. Therefore, for non linear regularization the bifurcation diagram depends strongly of the transition map $\varphi$.
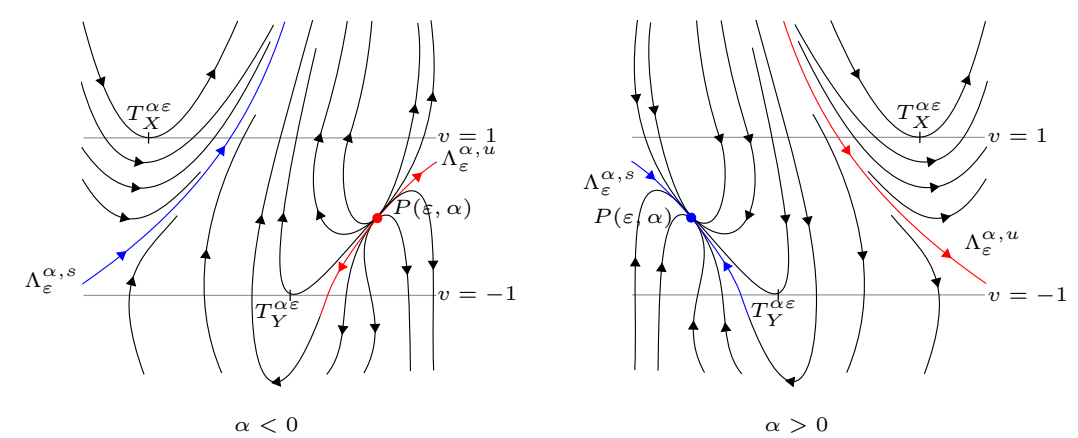

Figure 22: The phase portrait of $Z_{\varepsilon}^{\alpha}$ for $(\alpha, \varepsilon)$ below the curve $\mathcal{D}$.

Proposition 4.6. Let $Z \in \Lambda^{F}$ having a visible-invisible fold, satisfying $X^{1} \cdot Y^{1}(\mathbf{0})<0$ and $(\operatorname{det} Z)_{x}(\mathbf{0})<0$. Then, for $\alpha=\delta \varepsilon$ the stable and unstable Fenichel manifolds $\Lambda_{\varepsilon}^{\alpha, s}$ and $\Lambda_{\varepsilon}^{\alpha, u}$ of the regularized system $Z_{\varepsilon}^{\alpha}$ can be extended up to $x=0$. Moreover:

$$
\Lambda_{\varepsilon}^{\alpha, s, u} \cap\{x=0\}=\left(0, \bar{v}+\mathcal{O}\left(\varepsilon^{\frac{1}{2}}\right)\right),
$$

where $\bar{v}=m_{0}(0)$ is given in Equation (3.26), and the system has a maximal Canard for

$$
\mathcal{C}=\left\{(\alpha, \varepsilon): \alpha=\alpha_{\mathcal{C}}(\varepsilon)=\delta_{\mathcal{C}} \varepsilon+\mathcal{O}\left(\varepsilon^{\frac{3}{2}}\right)\right\}
$$

and $\varepsilon$ small enough, where $\delta_{C}=-\frac{M_{0} M_{3}+M_{1} M_{4}}{M_{2} M_{4}}$ and the constants $M_{i}$ are given by

$$
\begin{aligned}
& M_{0}=\left(X^{1}+Y^{1}+\varphi(\bar{v})\left(X^{1}-Y^{1}\right)\right)(\mathbf{0}), \\
& M_{1}=\bar{v}\left(X_{y}^{2}+Y_{y}^{2}+\varphi(\bar{v})\left(X_{y}^{2}-Y_{y}^{2}\right)\right)(\mathbf{0}), \\
& M_{2}=\left(\tilde{X}^{2}+\tilde{Y}^{2}+\varphi(\bar{v})\left(\tilde{X}^{2}-\tilde{Y}^{2}\right)\right)(\mathbf{0}), \\
& M_{3}=\frac{1}{2}\left(X_{x x}^{2}+Y_{x x}^{2}+\varphi(\bar{v})\left(X_{x x}^{2}-Y_{x x}^{2}\right)\right)(\mathbf{0}), \\
& M_{4}=\varphi^{\prime}(\bar{v})\left(X_{x}^{2}-Y_{x}^{2}\right)(\mathbf{0}) .
\end{aligned}
$$

Moreover, for $\alpha=\alpha_{\mathcal{C}}(\varepsilon)$, one has that $\Lambda_{\varepsilon}^{\alpha, s} \cap\{x=0\}=\Lambda_{\varepsilon}^{\alpha, u} \cap\{x=0\}=(0, \bar{v}+\mathcal{O}(\varepsilon))$,

Proof. The proof of this proposition is done using asymptotic methods and it is deferred to subsection 7.2 in the appendix.

Next, we will see how the maximal Canard obtained in Proposition 4.6 plays an important role in the behavior of the periodic orbits of the system.

During this section, we fix $\mathcal{C}<\mathcal{H}$, that is, the curve $\mathcal{C}$ where the Canard trajectory takes place is located to the left of the curve $\mathcal{H}$ where the Hopf bifurcation happens. The other case can be done analogously. In Theorems 4.7 and 4.8, completed by Proposition 4.11 we present the bifurcation diagram of the regularized system $Z_{\varepsilon}^{\alpha}$ depending on the sign of the first Lyapunov coefficient $\ell_{1}(\alpha(\varepsilon), \varepsilon)$ over the curve $\mathcal{H}$ and the sign of the "way-in,way-out" function (4.12). 


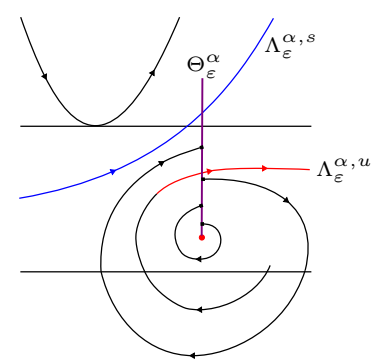

(a) $\alpha<\alpha_{\mathcal{C}}$

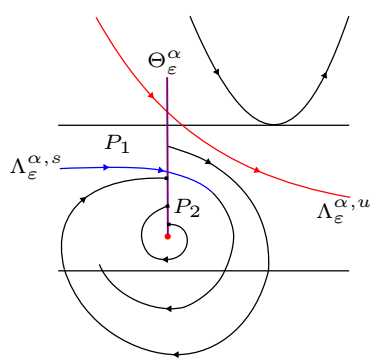

(b) $\alpha_{\mathcal{C}}<\alpha<\alpha_{\mathcal{H}}$

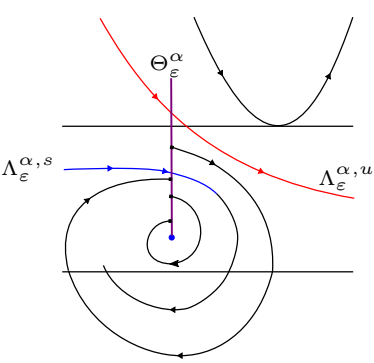

(c) $\alpha>\alpha_{\mathcal{H}}$

Figure 23: The first return map associated to the regularized system $Z_{\varepsilon}^{\alpha}$ when the folds have opposite visibility.

Theorem 4.7. Let $Z \in \Lambda^{F}$ having an visible-invisible fold-fold singularity at the origin satisfying $X^{1} \cdot Y^{1}(\mathbf{0})<0$ and $(\operatorname{det} Z)_{x}(\mathbf{0})<0$. Suppose that on the curve $\mathcal{H}$ (given in (3.16)), the first Lyapunov coefficient $\ell_{1}(\alpha(\varepsilon), \varepsilon)<0$ and for each $\varepsilon$ sufficiently small $\alpha_{\mathcal{C}}(\varepsilon)<\alpha_{\mathcal{H}}(\varepsilon)$.

Let $\alpha_{\mathcal{D}}^{ \pm}\left(\varepsilon_{0}\right), \alpha_{\mathcal{H}}\left(\varepsilon_{0}\right)$ and $\alpha_{\mathcal{C}}\left(\varepsilon_{0}\right)$ be the intersection of the curves $\mathcal{D}^{ \pm} \overline{(3.15)}, \mathcal{H}(\overline{(3.16)})$ and $\mathcal{C}\left(\right.$ (7.30) with the line $\varepsilon=\varepsilon_{0}$, respectively. We have the following:

- For $\alpha<\alpha_{\mathcal{D}}^{-}\left(\varepsilon_{0}\right)$ the critical point $P(\alpha, \varepsilon)$ is a unstable node;

- For $\alpha_{\mathcal{D}}^{-}\left(\varepsilon_{0}\right)<\alpha<\alpha_{\mathcal{C}}\left(\varepsilon_{0}\right)$ the critical point $P(\alpha, \varepsilon)$ is a unstable focus;

- For $\alpha=\alpha_{\mathcal{C}}\left(\varepsilon_{0}\right)$ the stable and unstable Fenichel manifolds of system $Z_{\varepsilon}^{\alpha}$ coincide along a maximal Canard and there exists an stable periodic orbit $\Delta_{\varepsilon}^{\alpha, s}$ for $\alpha>\alpha_{\mathcal{C}}\left(\varepsilon_{0}\right)$.

- The periodic orbit $\Delta_{\varepsilon}^{\alpha, s}$ persists for $\alpha_{\mathcal{C}}<\alpha<\alpha_{\mathcal{H}}\left(\varepsilon_{0}\right)$.

- For $\alpha=\alpha_{\mathcal{H}}\left(\varepsilon_{0}\right)$ a supercritical Hopf bifurcation takes place. The critical point $P(\alpha, \varepsilon)$ becomes an stable focus.

- Moreover, the critical point $P(\alpha, \varepsilon)$ is a stable focus for $\alpha_{\mathcal{H}}\left(\varepsilon_{0}\right)<\alpha<\alpha_{\mathcal{D}}^{+}\left(\varepsilon_{0}\right)$ and a stable node $\alpha>\alpha_{\mathcal{D}}^{+}\left(\varepsilon_{0}\right)$.

- If the Melnikov function $M(v, \delta)$ is strictly concave for $\delta$ near $\delta_{\mathcal{H}}$, then the periodic orbit $\Delta_{\varepsilon}^{\alpha, s}$ is unique and disappears at $\alpha=\alpha_{\mathcal{H}}\left(\varepsilon_{0}\right)$.

Proof. The character of the critical point is given by Corolary 3.8. The dynamics for $(\alpha, \varepsilon)$ below the parabola $\mathcal{D}$ has been discussed in the beginning of this section. We are going focus on the dynamics above the parabola $\mathcal{D}$.

When $\alpha_{\mathcal{D}}\left(\varepsilon_{0}\right)^{-}<\alpha<\alpha_{\mathcal{H}}\left(\varepsilon_{0}\right)$ the critical point $P(\alpha, \varepsilon)$ is a unstable focus, see Fig. 25(a)

In the case $\alpha_{\mathcal{C}}\left(\varepsilon_{0}\right)<\alpha_{\mathcal{H}}\left(\varepsilon_{0}\right)$ for each fixed $\varepsilon$ small enough, the maximal Canard occurs before the Hopf bifurcation.

For $\alpha<0$ and $\varepsilon>0$ sufficiently small, the stable Fenichel manifold $\Lambda_{\varepsilon}^{\alpha, s}$ becomes unbounded for positive time. The same occurs for $\alpha>0$ for the unstable Fenichel manifold $\Lambda_{\varepsilon}^{\alpha, u}$ in negative time, see Figure 22

When $\alpha=\delta \varepsilon$, the critical manifold $\Lambda_{0}^{\alpha}$ associated to the vector field $Z_{\varepsilon}^{\alpha}$ is equal to the critical manifold $\Lambda_{0}=\Lambda_{0}^{s} \cup \Lambda_{0}^{u}$ associated to the vector field $Z_{\varepsilon}^{\alpha}, \alpha=0$. therefore, when $\alpha \rightarrow \alpha_{\mathcal{C}}\left(\varepsilon_{0}\right)^{ \pm}$, both the stable and unstable the Fenichel manifolds become "flattened" until they coincide at $\alpha=\alpha_{\mathcal{C}}\left(\varepsilon_{0}\right)$, see Fig. 24(b) However, by continuity, for $\alpha<\alpha_{\mathcal{C}}\left(\varepsilon_{0}\right)$ the stable Fenichel manifold is above the unstable one and the opposite occurs for $\alpha>\alpha_{\mathcal{C}}\left(\varepsilon_{0}\right)$.

At the point $\alpha=\alpha_{\mathcal{C}}\left(\varepsilon_{0}\right)$, the manifolds $\Lambda_{\varepsilon}^{\alpha, u}$ and $\Lambda_{\varepsilon}^{\alpha, s}$ coincide as in Fig. 24(b) and the unstable focus $P(\alpha, \varepsilon)$ is below them (see Remark 3.13). This change on the relative position of the critical manifolds $\Lambda_{\varepsilon}^{\alpha, s}$ and $\Lambda_{\varepsilon}^{\alpha, u}$, gives raise to a stable periodic orbit which persists for $\alpha_{\mathcal{C}}\left(\varepsilon_{0}\right)<\alpha<$ $\alpha_{\mathcal{H}}\left(\varepsilon_{0}\right)$.

In fact, let $\Theta$ be a cross section transverse to the stable Fenichel manifold $\Lambda_{\varepsilon}^{\alpha, s}$ and containing the point $P(\alpha, \varepsilon)$, consider the return map $\pi: \Theta \rightarrow \Theta$. Take $P_{1} \in \Theta$ which is above and sufficiently 


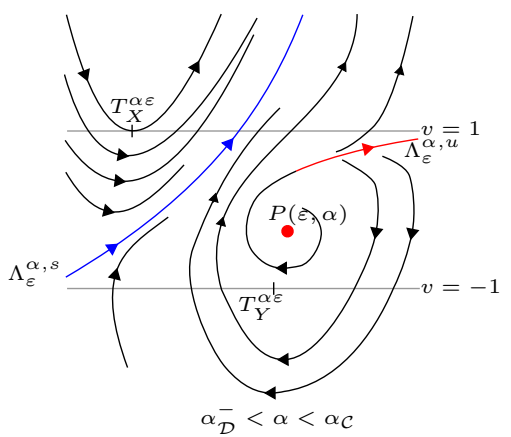

(a)

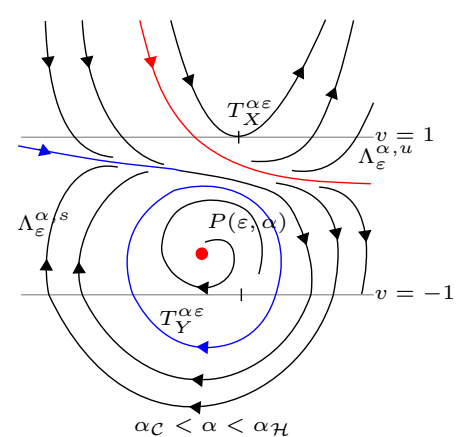

(c)

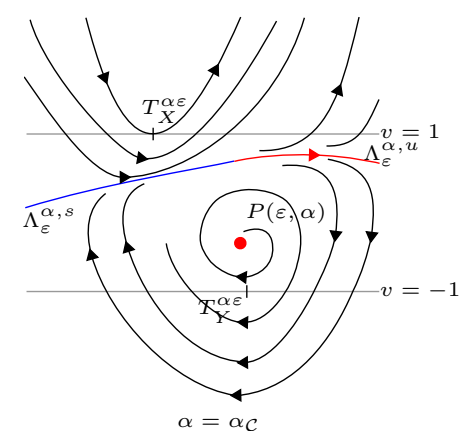

(b)

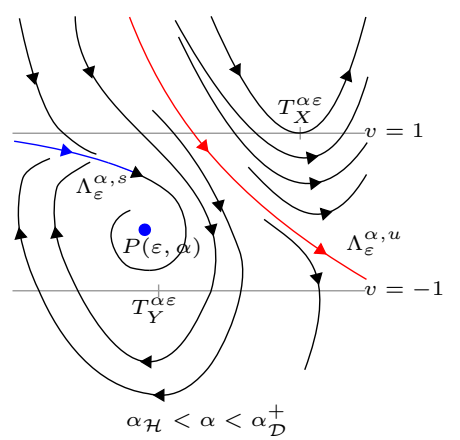

(d)

Figure 24: The supercritical Hopf bifurcation on the regularization of a visible-invisible fold-fold satisfying $\alpha_{\mathcal{C}}<\alpha_{\mathcal{H}}$.

close to $\Lambda_{\varepsilon}^{\alpha, s}$, its trajectory crosses the line $v=-1$ and then intersects the section $\Theta$ below $\Lambda_{\varepsilon}^{\alpha, s}$; therefore $\pi\left(P_{1}\right)-P_{1}<0$. Opposed to this behavior, since the point $P(\alpha, \varepsilon)$ is a unstable focus, the trajectory of any initial condition $P_{2} \in \Theta$ near the focus intersects, for negative time, $\Theta$ even closer to the focus point $P(\alpha, \varepsilon)$, see Figure 23 therefore $\pi\left(P_{2}\right)-P_{2}>0$.

This way, the Bolzano-Weierstrass Theorem, guarantees the existence of a fixed point of the return map $\pi$ which gives an stable periodic orbit $\Delta_{\varepsilon}^{\alpha, s}$ for all $\alpha_{\mathcal{C}}\left(\varepsilon_{0}\right)<\alpha<\alpha_{\mathcal{H}}\left(\varepsilon_{0}\right)$.

When $\alpha=\alpha_{\mathcal{H}}\left(\varepsilon_{0}\right)$, the critical point $P(\alpha, \varepsilon)$ undergoes to a supercritical Hopf bifurcation, therefore, for $\alpha<\alpha_{\mathcal{H}}\left(\varepsilon_{0}\right)$ there exists a small stable periodic orbit $\bar{\Delta}_{\varepsilon}^{\alpha, s}$ near the critical point $P(\alpha, \varepsilon)$. For $\alpha>\alpha_{\mathcal{H}}\left(\varepsilon_{0}\right)$ the critical point changes its stability and becomes a stable focus, therefore, there are no periodic orbits near the critical point.

On the other hand, if the Melnikov function $M(v, \delta)$ is strictly concave for $\delta$ near $\delta_{\mathcal{C}}$ and $\alpha=\delta \varepsilon$, system $Z_{\varepsilon}^{\alpha}$ has a unique periodic orbit for parameter values in this interval. Therefore it follows that the two stable periodic orbits $\Delta_{\varepsilon}^{\alpha, s}$ and $\bar{\Delta}_{\varepsilon}^{\alpha, s}$ coincide. This means that, in this case, the periodic orbit which raises from the canard becomes smaller until it disappear in the Hopf bifurcation. Finally, for $\alpha>\alpha_{\mathcal{H}}\left(\varepsilon_{0}\right)$ the regularized system has no periodic orbits.

Theorem 4.8. Consider the same hypothesis of Theorem 4.7 but now $\ell_{1}(\alpha(\varepsilon), \varepsilon)>0$.

Let $\alpha_{\mathcal{D}}^{ \pm}\left(\varepsilon_{0}\right), \alpha_{\mathcal{H}}\left(\varepsilon_{0}\right)$ and $\alpha_{\mathcal{C}}\left(\varepsilon_{0}\right)$ be the intersection of the line $\varepsilon=\varepsilon_{0}$ with the curves $\mathcal{D}^{ \pm}, \mathcal{H}$ and $\mathcal{C}$, respectively. Generically, we have the following

- For $\alpha<\alpha_{\mathcal{D}}^{-}\left(\varepsilon_{0}\right)$ the critical point $P(\alpha, \varepsilon)$ is a unstable node;

- For $\alpha_{\mathcal{D}}\left(\varepsilon_{0}\right)^{-}<\alpha<\alpha_{\mathcal{H}}\left(\varepsilon_{0}\right)$ the critical point $P(\alpha, \varepsilon)$ is a unstable focus;

- When $\alpha=\alpha_{\mathcal{C}}\left(\varepsilon_{0}\right)$ the system $Z_{\varepsilon}^{\alpha}$ has a Canard trajectory;

- For $\alpha_{\mathcal{C}}\left(\varepsilon_{0}\right)<\alpha<\alpha_{\mathcal{H}}\left(\varepsilon_{0}\right)$ there exists a stable periodic orbit $\Delta_{\varepsilon}^{\alpha, s}$ and the critical point $P(\alpha, \varepsilon)$ is a unstable focus;

- When $\alpha=\alpha_{\mathcal{H}}\left(\varepsilon_{0}\right)$ a subcritical Hopf bifurcation takes place. The critical point $P(\alpha, \varepsilon)$ becomes a stable focus and a unstable periodic orbit $\Delta_{\varepsilon}^{\alpha, u}$ appears; 
- The pair of periodic orbits coexist for $\alpha_{\mathcal{H}}\left(\varepsilon_{0}\right)<\alpha<\alpha_{\mathcal{S}}\left(\varepsilon_{0}\right)$;

- When $\alpha>\alpha_{\mathcal{S}}\left(\varepsilon_{0}\right)$ there are no periodic orbits. Moreover, the critical point $P(\alpha, \varepsilon)$ is an stable focus when $\alpha_{\mathcal{H}}\left(\varepsilon_{0}\right)<\alpha<\alpha_{\mathcal{D}}^{+}\left(\varepsilon_{0}\right)$ and an stable node for $\alpha>\alpha_{\mathcal{D}}^{+}\left(\varepsilon_{0}\right)$.

Proof. The character of the critical point and the argument which gives the existence of the stable periodic orbit $\Delta_{\varepsilon}^{\alpha, s}$ for $\alpha_{\mathcal{C}}<\alpha<\alpha_{\mathcal{H}}$ is already proved in Theorem 4.7, see Fig. 25(c),

At $\alpha>\alpha_{\mathcal{H}}\left(\varepsilon_{0}\right)$, the point $P(\alpha, \varepsilon)$ becomes an stable focus. Since the Hopf bifurcation is subcritical $\left(\ell_{1}(\alpha(\varepsilon), \varepsilon)>0\right)$, a unstable periodic orbit $\Delta_{\varepsilon}^{\alpha, u}$ appears for $\alpha>\alpha_{\mathcal{H}}\left(\varepsilon_{0}\right)$, as shown in Fig. 25(d). Observe that the stable periodic orbit $\Delta_{\varepsilon}^{\alpha, s}$ persistence is guaranteed by the first return map and therefore, both periodic orbits coexist.

Since for fixed $\alpha>0$ and $\varepsilon>0$ small enough, $Z_{\varepsilon}^{\alpha}$ has no periodic orbits, for each $\varepsilon$ it must exist a value of $\alpha_{\mathcal{S}}\left(\varepsilon_{0}\right)$ such that the two orbits collapse and then disappear, as illustrated in Figures $25(\mathrm{e})$ and 25(f), respectively. One expects that, in the simplest case, for $\alpha=\alpha_{\mathcal{S}}\left(\varepsilon_{0}\right)$ the periodic orbits collide in a saddle-node bifurcation.

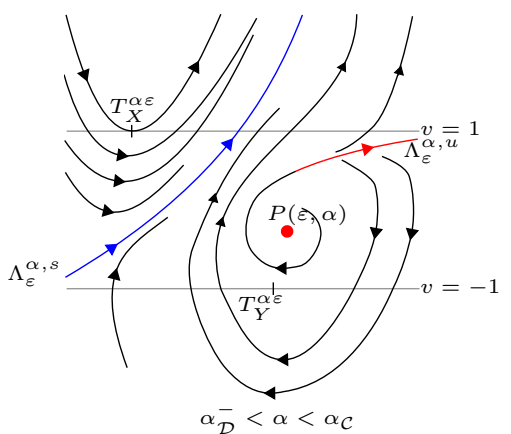

(a)

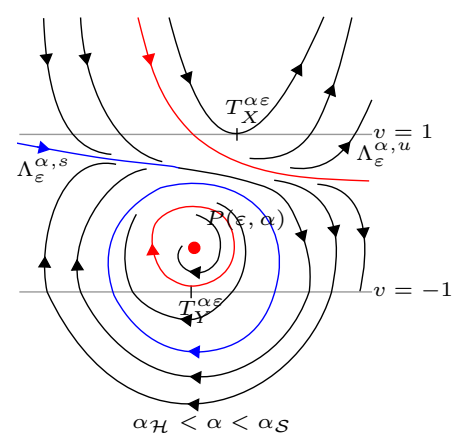

(d)

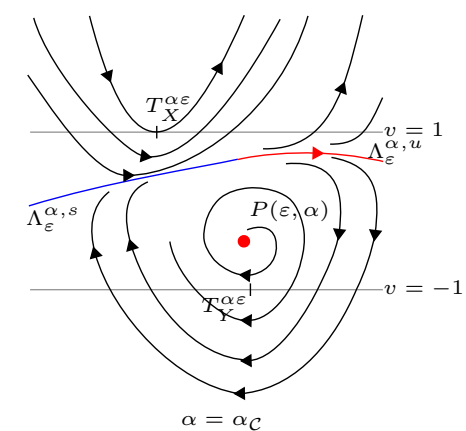

(b)

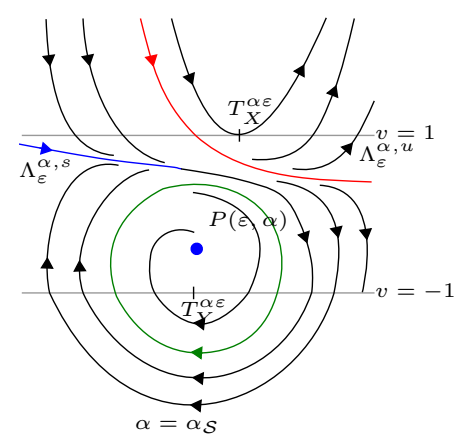

(e)

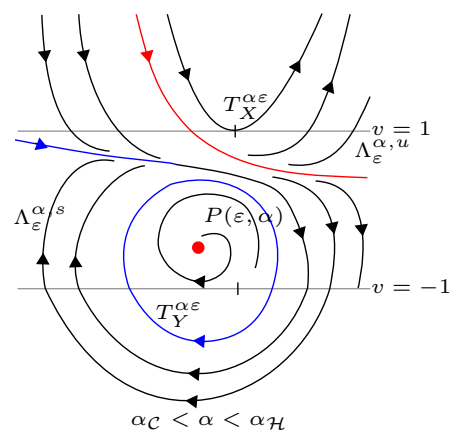

(c)

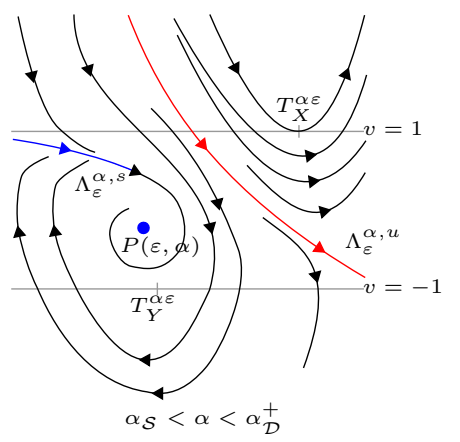

(f)

Figure 25: The bifurcation diagram for each fixed $\varepsilon_{0}>0$ when $P(\alpha, \varepsilon)$ suffers a subcritical Hopf bifurcation and $\alpha_{\mathcal{H}}(\varepsilon)>\alpha_{\mathcal{C}}(\varepsilon)$.

Remark 4.9. In this case one can obtain a proposition similar to Proposition 4.5 which gives the condition for the periodic orbits to collide in a saddle-node bifurcation and, when it exists, the value of the saddle node bifurcation parameters using the Melnikov function $M(v, \delta)$ given in (5.3).

Theorems 4.7 and 4.8 show that the regularization of the unfolding of a visible-invisible fold with $(\operatorname{det} Z)_{x}(\mathbf{0})<0$ and $\alpha_{\mathcal{C}}<\alpha_{\mathcal{H}}$ behaves very closely to the classical slow-fast systems studied by Krupa and Szmolyan in KS01a. Next theorem shows that, when the transition function is linear, both systems are $\mathcal{C}^{r}$-conjugated inside the regularization zone.

Theorem 4.10 (Linear regularization). Let $Z$ having a visible-invisible fold-fold point at the origin satisfying $X^{1} \cdot Y^{1}(\mathbf{0})<0$ and $(\operatorname{det} Z)_{x}(\mathbf{0})<0$. Consider $Z^{\alpha}$ an unfolding of $Z$ and $Z_{\varepsilon}^{\alpha}$ its regularization with a linear transition function: $\varphi(v)=v$ for $v \in(-1,1)$. In addition, suppose that $\alpha=\delta \varepsilon$. Then there exists a change of variables which transforms system (3.5) in the region 
$|v| \leq 1$ into the general slow-fast system (7.32), As a consequence the maximal Canard occurs for $\alpha_{\mathcal{C}}=\delta_{\mathcal{C}} \varepsilon+\mathcal{O}\left(\varepsilon^{3 / 2}\right)$

$$
\delta_{\mathcal{C}}=\delta_{\mathcal{H}}+\bar{A},
$$

with

$$
\bar{A}=-\frac{1}{N(Z, \tilde{Z})}\left(-\frac{A_{10} A_{3}}{A_{6}^{2}}+\frac{A_{9}}{A_{6}}-A_{1}\right) .
$$

which has the same sign as the Lyapunov coefficient $\ell_{1}(\alpha(\varepsilon), \varepsilon)$ at the point $P(\alpha, \varepsilon)$.

Proof. The proof of this proposition is deferred to subsection 7.3 in the appendix.

Consequently, when $\varphi$ is linear, if $\delta_{\mathcal{C}}<\delta_{\mathcal{H}}$, we have that $\ell_{1}<0$ and therefore the hypothesis of Theorem 4.8 can not be fulfilled. Consequently the dynamics of the regularized system $Z_{\varepsilon}^{\alpha}$ in this case is always given by Theorem 4.7. One could think that it would be possible to state an analogous result for the regularized system $Z_{\varepsilon}^{\alpha}$ with a nonlinear transition map. However, in Example 4.4 using the cubic transition map (4.5), we show that in these cases the dynamics is not always equivalent to the Krupa-Szmolyan system.

\subsubsection{The function $R$ : disappearance of the periodic orbit after the occurrence of the maximal Canard}

In this section we give a description of how the stable periodic orbit $\Delta_{\varepsilon}^{\alpha, s}$ obtained in Theorems 4.8 and 4.7. and which exists for $\alpha>\alpha_{\mathcal{C}}$, disappears after the maximal Canard occurs for $\alpha<\alpha_{\mathcal{C}}$. We present here an argument different from one used in these theorems, and which is independent of the character of the critical point $P(\alpha, \varepsilon)$, which shows that, exponentially close (respect to $\varepsilon$ ) to the parameter value $\alpha=\alpha_{\mathcal{C}}(\varepsilon)$, a "big" (of order $\mathcal{O}\left(\frac{1}{\varepsilon}\right)$ in the $(x, v)$ plane) periodic orbit $\Delta_{\varepsilon}^{\alpha, \mathcal{C}}$ exists. The different mechanisms that make the stable orbit $\Delta_{\varepsilon}^{\alpha, s}$ "disappear" depend on the (attracting or repelling) character of $\Delta_{\varepsilon}^{\alpha, \mathcal{C}}$.

The existence of the big periodic orbit $\Delta_{\varepsilon}^{\alpha, \mathcal{C}}$ is also shown in KH15b using the ideas of [KS01b], but we present it here because our argument is different. As usually happens in singular perturbed problems (see Eck83 KS01b]), periodic orbits of size $\mathcal{O}(1)$ in the variable $y\left(\mathcal{O}\left(\frac{1}{\varepsilon}\right)\right.$ in the variable $v$ ) exist when the parameter $\alpha$ is close to the value $\alpha_{\mathcal{C}}=\delta_{\mathcal{C}} \varepsilon+\mathcal{O}\left(\varepsilon^{3 / 2}\right)$ where the maximal Canard exists, in fact, exponentially close.

The reasoning which gives the existence of these "big" periodic orbits will be made in the original variables $(x, y)$ of the problem and is the following. Take $y^{*}<0$, small but independent of $\varepsilon$. Consider $\alpha=\delta \varepsilon$, the section $\Theta=\left\{(0, y), y \leq y^{*}\right\}$ and $\Theta_{\varepsilon}=\{(0, y), y \leq \varepsilon\}$ and consider the maps $\pi^{s}, \pi^{u}(\cdot ; \delta): \Theta \rightarrow \Theta_{\varepsilon}$, which follow the flow in positive or negative times until it meets the section $\Theta_{\varepsilon}$. These maps are well defined if the Fenichel manifolds are close enough, therefore for $\alpha=\delta \varepsilon$ close enough to $\alpha_{\mathcal{C}}$.

Fix $y \in \Theta$. Depending of the position of the Fenichel manifolds, which depends on the sign of $\delta-\delta_{\mathcal{C}}$, the sign of $f(\delta)=\pi^{s}(y ; \delta)-\pi^{u}(y ; \delta)$ changes (see Figure 26). By Bolzano theorem it must exist a value of $\delta=\delta(y ; \varepsilon)$ such that $f(\delta)=\pi^{s}(y ; \delta)-\pi^{u}(y ; \delta)=0$, and therefore a periodic orbit $\Delta_{\varepsilon}^{\alpha, \mathcal{C}}$ of system (3.5) passing through $\left(0, \frac{y}{\varepsilon}\right)$ exists for this value of $\delta$. Moreover, calling $\Lambda_{\varepsilon}^{\alpha, s, u} \cap\{x=0\}=v^{s, u}$, for $\delta=\delta(y ; \varepsilon)$ :

$$
0=f(\delta)=\pi^{s}(y ; \delta)-\pi^{u}(y ; \delta)=\pi^{s}(y ; \delta)-y^{s}(\delta)+y^{s}(\delta)-y^{u}(\delta)+y^{u}(\delta)-\pi^{u}(y ; \delta)
$$

where $y^{s, u}=v^{s, u} \varepsilon$. Due to the exponential attraction of the Fenichel manifolds, we know that $\pi^{s / u}(y ; \delta)-y^{s / u}(\delta)=\varepsilon \mathcal{O}\left(e^{-\frac{c(y)}{\varepsilon}}\right)$. Using that $y^{s}(\delta)-y^{u}(\delta)=\varepsilon^{3 / 2} C\left(\delta-\delta_{\mathcal{C}}\right)+\mathcal{O}\left(\varepsilon^{2}\right)$, (see Equation (7.31) we obtain that, for $\varepsilon$ small enough:

$$
\delta(y ; \varepsilon)=\delta_{\mathcal{C}}+\mathcal{O}\left(e^{-\frac{c(y)}{\varepsilon}}\right), \quad c(y)>0 .
$$

The orbit $\Delta_{\varepsilon}^{\alpha, \mathcal{C}}$ which arises from the point $(0, v)=\left(0, \frac{y}{\varepsilon}\right)$ is given, in first order, by the singular orbit having four pieces: $\Delta=\Delta_{1} \cup \Delta_{2} \cup \Delta_{3} \cup \Delta_{4}$. 

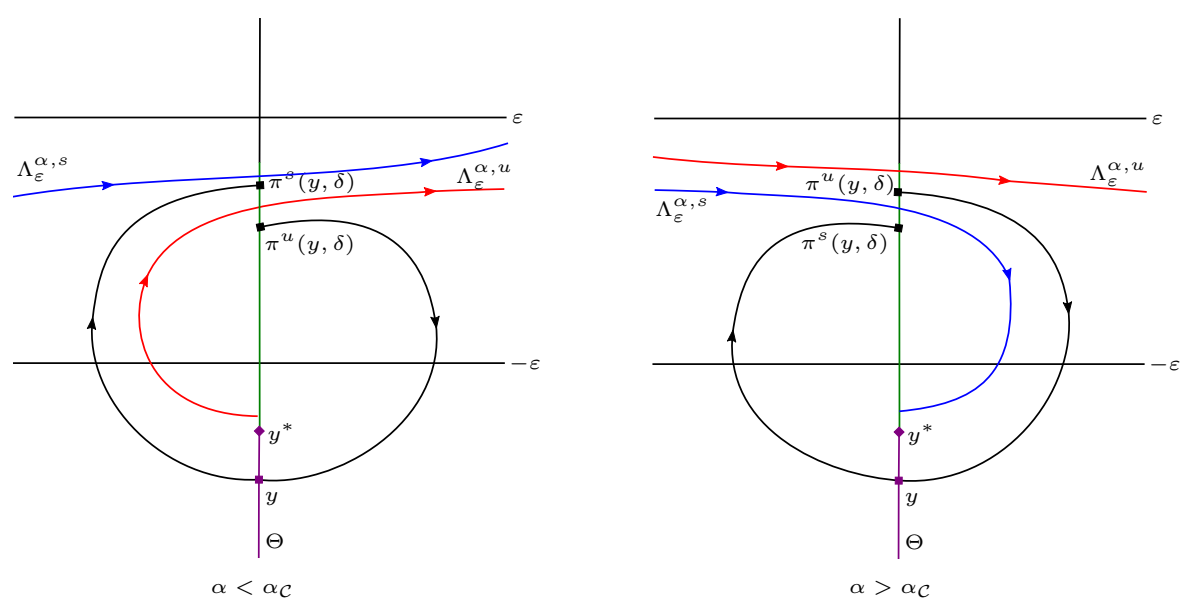

Figure 26: The maps $\pi u, s(y, \delta)$ for $\alpha=\delta \varepsilon$ close to the value $\alpha=\alpha_{\mathcal{C}}$

- $\Delta_{1}$ is the orbit through $(0, y)$ of the vector field $Y$ written in $(x, v)=\left(x, \frac{y}{\varepsilon}\right)$ :

$$
\Delta_{1}=\left\{\varphi_{Y}(t ; 0, y),-t_{1} \leq t \leq t_{2}\right\}, \text { where } \varphi_{Y}\left(-t_{1} ; 0, y\right)=(x, 0), \varphi_{Y}\left(t_{2} ; 0, y\right)=(\bar{x}, 0)
$$

where $\varphi_{Y}$ denotes the flow of the vector field $Y$.

- $\Delta_{2}$ is the vertical orbit through $(\bar{x},-1)$ :

$$
\Delta_{2}=\{(\bar{x}, v(t))\} \text {, where } \varepsilon v^{\prime}(t)=F^{2}(\bar{x}, v(t) ; 0,0), t_{2} \leq t \leq t_{2}+\varepsilon \bar{t}:=t_{3}
$$

- $\Delta_{3}$ is the piece of maximal Canard between the points $\left(x, m_{0}(x)\right)$ and $\left(\bar{x}, m_{0}(\bar{x})\right)$ (see Equation (3.26) :

$$
\Delta_{3}=\left\{\left(x(t), m_{0}(x(t))\right\}, \text { where } x^{\prime}(t)=F^{1}\left(x(t), m_{0}(x(t)) ; 0,0\right), t_{3} \leq t \leq t_{4} .\right.
$$

- $\Delta_{4}$ is the vertical orbit through $(x, \bar{v})$ :

$$
\Delta_{4}=\{(x, v(t))\}, \text { where } \varepsilon v^{\prime}(t)=F^{2}(x, v(t) ; 0,0), t_{4} \leq t \leq t_{4}+\varepsilon \tilde{t}:=t_{5}
$$

The stability of the periodic orbit $\Delta_{\varepsilon}^{\alpha, \mathcal{C}}$ arising from $\Delta$ is given by:

$$
\int_{0}^{T} \operatorname{Div} F\left(\Delta_{\varepsilon}^{\alpha, \mathcal{C}}(t), \delta \varepsilon, \varepsilon\right) d t
$$

where $T=T(\varepsilon)$ is its period. Using the form of the equations and the fact that $\varphi(v)=-1$ for $v \leq-1$, this integral is given in first order respect to $\varepsilon$ by the so called "way-in, way-out" function:

$$
\frac{1}{\varepsilon} R=\frac{1}{\varepsilon} \int_{t_{3}}^{t_{4}} \varphi^{\prime}\left(m_{0}(x(t))\left(X^{2}-Y^{2}\right)(x(t), 0) d t .\right.
$$

which correspond to the integration along the "Canard piece" $\Delta_{3}$. It is important to mention that, even if our system is not slow fast for $v \leq-1$ (it is simply the vector field $Y$ written in variables $\left.\left(x, v=\frac{y}{\varepsilon}\right)\right)$, and the time spent in $\Delta_{1}$ is of the same order that the one in $\Delta_{3}$, the fact that $\varphi$ is constant for $v \leq-1$ makes the classical "way-in, way-out" function be also the dominant term of this integral.

Changing variables in the integral $s=x(t)$, and using that $d s=F^{1}\left(x(t), m_{0}(x(t)) ; 0,0\right) d t$ and that $\bar{x}=\phi_{Y}(x)$, where $\phi_{Y}$ is the Poincaré map associated to the vector field $Y$ near its invisible fold given in Proposition 2.12, one obtains a suitable form for this function, parameterized by the coordinate $x$ :

$$
R(x)=-\int_{\phi_{Y}(x)}^{x} \varphi^{\prime}\left(m_{0}(s)\right) \frac{\left(X^{2}-Y^{2}\right)^{2}}{2(\operatorname{det} Z)}(s, 0) d s .
$$


Note that in [KS01b the authors parametrize this function by the "hight" of the periodic orbit that in our case corresponds to $y=\mathcal{O}\left(x^{2}\right)$.

For $0<x<1$, using that $\phi_{Y}(x)=-x+\beta_{Y} x^{2}+O\left(x^{3}\right)$, as given in Proposition 2.12, the Taylor expansion of $R(x)$ is:

$$
R(x)=-\left(\varphi^{\prime}(\bar{v}) G^{\prime}(0) \beta_{Y}+\frac{2}{3} \varphi^{\prime \prime}(\bar{v}) m_{0}^{\prime}(0) G^{\prime}(0)+\frac{\varphi^{\prime}(\bar{v})}{3} G^{\prime \prime}(0)\right) x^{3}+O\left(x^{4}\right) .
$$

where $G(x)=\frac{\left(X^{2}-Y^{2}\right)^{2}}{2(\operatorname{det} Z)}(x, 0)$. Using the expression for $m_{0}(x)$ given in $(3.26)$ and $G(x)$ one obtains:

$$
R(x)=A x^{3}+O\left(x^{4}\right)
$$

with

$$
A=-\frac{G^{\prime}(0)}{3}\left[\varphi^{\prime}(\bar{v})\left\{3 \beta_{Y}+2\left(\frac{X_{x x}^{2}-Y_{x x}^{2}}{X_{x}^{2}-Y_{x}^{2}}-\frac{(\operatorname{det} Z)_{x x}}{2(\operatorname{det} Z)_{x}}\right)\right\}+\frac{2 \varphi^{\prime \prime}(\bar{v})}{\varphi^{\prime}(\bar{v})} \frac{X_{x x}^{2} Y_{x}^{2}-Y_{x x}^{2} X_{x}^{2}}{\left(Y_{x}^{2}-X_{x}^{2}\right)^{2}}\right](\mathbf{0})
$$

and $G^{\prime}(0)=\frac{\left(X_{x}^{2}-Y_{x}^{2}\right)^{2}}{2(\operatorname{det} Z)_{x}}(\mathbf{0})<0$

Then, the periodic orbit $\Delta_{\varepsilon}^{\alpha, \mathcal{C}}$ of size $O(1)$ originated at the so called "Canard explosion" is stable if

$$
B=\left[\varphi^{\prime}(\bar{v})\left\{3 \beta_{Y}+2\left(\frac{X_{x x}^{2}-Y_{x x}^{2}}{X_{x}^{2}-Y_{x}^{2}}-\frac{(\operatorname{det} Z)_{x x}}{2(\operatorname{det} Z)_{x}}\right)\right\}+\frac{2 \varphi^{\prime \prime}(\bar{v})}{\varphi^{\prime}(\bar{v})} \frac{X_{x x}^{2} Y_{x}^{2}-Y_{x x}^{2} X_{x}^{2}}{\left(Y_{x}^{2}-X_{x}^{2}\right)^{2}}\right](\mathbf{0})<0
$$

and unstable otherwise. Moreover, the orbit $\Delta_{\varepsilon}^{\alpha, \mathcal{C}}$, for $\alpha=\delta(x) \varepsilon$ stays stable (unstable) while the function $R(x)$ stays negative (positive).

Next proposition tells us in what region of the parameter plane the periodic orbit $\Delta_{\varepsilon}^{\alpha, \mathcal{C}}$, which raises from the maximal Canard, appears. Moreover, it also explains the relation between the periodic orbit $\Delta_{\varepsilon}^{\alpha, \mathcal{C}}$ and the stable periodic orbit $\Delta_{\varepsilon}^{\alpha, s}$ given in Theorems 4.7 and 4.8 . Recall that the stable periodic orbit $\Delta_{\varepsilon}^{\alpha, s}$ obtained in these theorems exists for $(\alpha, \varepsilon)$ between the curves $\mathcal{C}$ and $\mathcal{H}$, independently of the sign of $B$.

Proposition 4.11. Let $B$ be the quantity given in (4.13), then:

- If $B<0$ the periodic orbit $\Delta_{\varepsilon}^{\alpha, \mathcal{C}}$ is stable and appears for $(\alpha, \varepsilon)$ located on the right of the curve $\mathcal{C}$. In addition, for $(\alpha, \varepsilon)$ on the left of the curve $\mathcal{C}$, for any compact set $\mathcal{K}$ containing the critical point $P(\alpha, \varepsilon)$, for $\varepsilon$ small enough, given an initial condition inside $\mathcal{K}$ then its trajectory tends to $P(\alpha, \varepsilon)$ backward in time and leaves $\mathcal{K}$ forward in time.

- If $B>0$ the periodic orbit $\Delta_{\varepsilon}^{\alpha, \mathcal{C}}$ is unstable and appears for $(\alpha, \varepsilon)$ located on the left of the curve $\mathcal{C}$. Moreover, for each $\varepsilon>0$ small enough, there exists a value $\alpha_{\tilde{\mathcal{S}}}$ satisfying $\alpha_{\mathcal{D}}^{-}(\varepsilon)<\alpha_{\tilde{\mathcal{S}}}(\varepsilon)<\alpha_{\mathcal{C}}(\varepsilon)$ such that

- For $\alpha_{\tilde{\mathcal{S}}}(\varepsilon)<\alpha<\alpha_{\mathcal{C}}(\varepsilon)$ the unstable periodic orbit $\Delta_{\varepsilon}^{\alpha, \mathcal{C}}$ coexists with the smaller stable periodic orbit $\Delta_{\varepsilon}^{\alpha, s}$ given in Theorems 4.7 and 4.8 .

- For $\alpha<\alpha_{\tilde{\mathcal{S}}}(\varepsilon)$ there are no periodic orbits.

Proof. At first, we prove the region of the parameter plane where the periodic orbit appears. Suppose $B<0$ and that $\Delta_{\varepsilon}^{\alpha, \mathcal{C}}$ exists for $(\alpha, \varepsilon)$ on the left of $\mathcal{C}$. Therefore, the Poincaré map defined in the cross section $\Theta$ (see Theorem 4.7) is repelling near the unstable manifold and attracting near the periodic orbit $\Delta_{\varepsilon}^{\alpha, \mathcal{C}}$. This reasoning guarantees the existence of a bigger unstable periodic $\tilde{\Delta}_{\varepsilon}^{\alpha, u}$ which is located below the unstable manifold and its interior contains $\Delta_{\varepsilon}^{\alpha, \mathcal{C}}$ which is a contradiction with $B<0$. The case $B>0$ can be proved analogously. The persistence of the periodic orbit $\Delta_{\varepsilon}^{\alpha, s}$ for $\alpha<\alpha_{\mathcal{C}}(\varepsilon)$ is given by the return map as we did in Theorem 4.8 using the repelling character of $\Delta_{\varepsilon}^{\alpha, \mathcal{C}}$, for an illustration see Figure 27. 

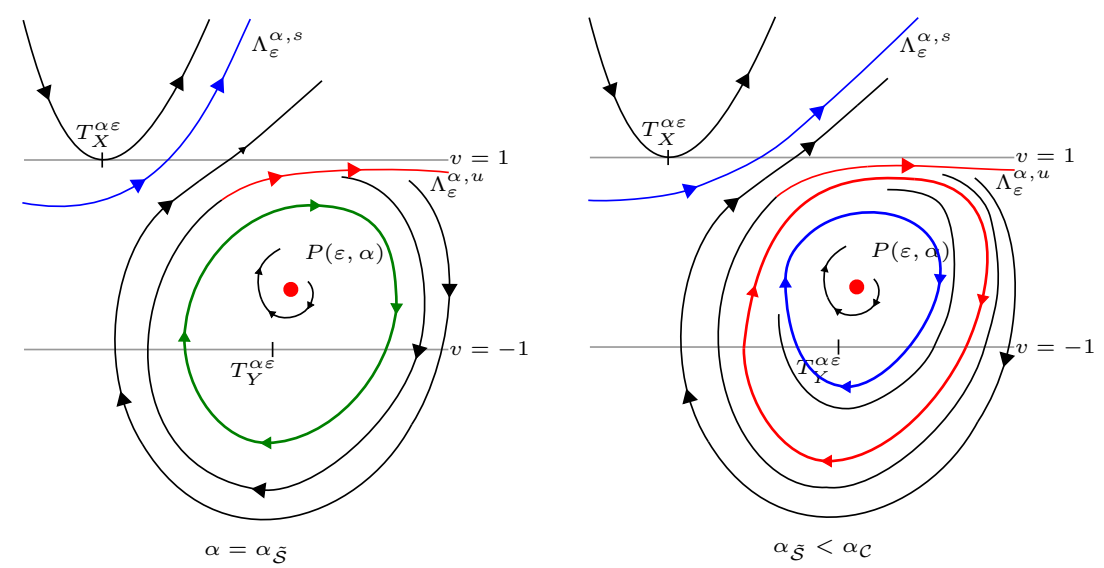

Figure 27: The periodic orbits of $Z_{\varepsilon}^{\alpha}$ for $\alpha<\alpha_{\mathcal{C}}$ when $B>0$. A saddle-node occurs at $\alpha=\alpha_{\tilde{\mathcal{S}}}$.

Remark 4.12. When $B<0$, using the information given in Theorem 4.7 we have two stable periodic orbits $\Delta_{\varepsilon}^{\alpha, C}$ and $\Delta_{\varepsilon}^{\alpha, s}$ for $\alpha$ near the value $\alpha_{\mathcal{C}}$. Therefore, in this situation, the simplest case is when these two periodic orbits coincide. Moreover, when $B>0$, since we have two periodic orbits $\Delta_{\varepsilon}^{\alpha, C}$ and $\Delta_{\varepsilon}^{\alpha, s}$ for $\alpha_{\tilde{\mathcal{S}}}<\alpha<\alpha_{\mathcal{C}}$ and no periodic for $\alpha<\alpha_{\tilde{\mathcal{S}}}$, the simplest case, is when the two periodic orbits collide in a saddle-node bifurcation for $\alpha=\alpha_{\tilde{\mathcal{S}}}$ and then disappear for $\alpha<\alpha_{\tilde{\mathcal{S}}}$.

The above discussion, joined to the results given in Theorems 4.7. 4.8 and Proposition 4.11. suggest that we have four simplest possibilities for the bifurcation diagram of $Z_{\varepsilon}^{\alpha}$ having a visibleinvisible fold with $(\operatorname{det} Z)_{x}(\mathbf{0})<0$. Moreover, if $R(x)<0 \forall x \geq 0$, then, for any $x>0$, one can find $\varepsilon$ small enough such that for $\delta=\delta(x ; \varepsilon)$ the stable periodic orbit $\Delta_{\varepsilon}^{\alpha, \mathcal{C}}$ exists until $\alpha=\delta(x ; \varepsilon) \varepsilon>$ $\alpha_{\mathcal{C}}(\varepsilon)$. The orbit increases unboundedly as $\alpha$ approaches $\alpha_{\mathcal{C}}(\varepsilon)$ and "disappears at infinity". This is the so-called Canard explosion. Nevertheless, due to the lack of compactness, fixing a value of $\varepsilon$ small enough so that all the previous results about the Fenichel manifolds are valid, we can only find the periodic orbit for $|x| \leq x^{*}(\varepsilon)$ and therefore for $\alpha$ close but until a certain distance of the $\alpha_{\mathcal{C}}(\varepsilon)$. Summarizing, the bifurcation diagram of $Z_{\varepsilon}^{\alpha}$ is then exactly as in Figures 24 or 25, depending on $\ell_{1}(\alpha, \varepsilon)$ sign.

However, if $B>0$, then we insert Figure 27 between subfigures $(a)$ and $(b)$ of Figures 24 and 25. depending on $\ell_{1}(\alpha, \varepsilon)$ sign. We summarize the four possible bifurcation diagram in Figure 28.

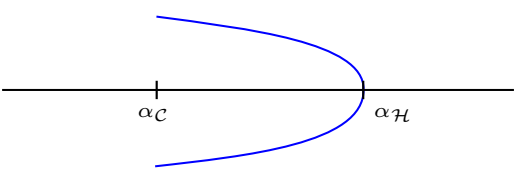

$R(x)<0$ and $\ell_{1}<0$

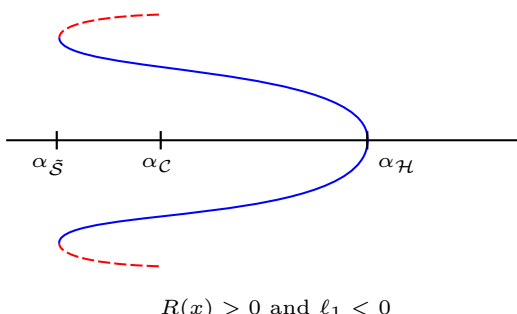

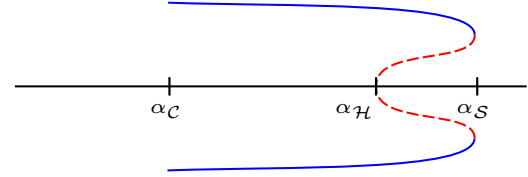

$R(x)<0$ and $\ell_{1}>0$

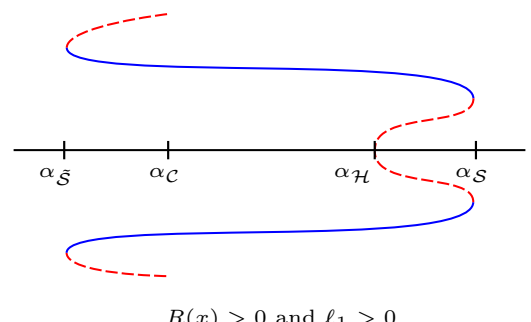

$R(x)>0$ and $\ell_{1}>0$

Figure 28: The four simplest- possible bifurcation diagrams for the periodic orbits of system $Z_{\varepsilon}^{\alpha}$ having a visibleinvisible fold-fold and satisfying $(\operatorname{det} Z)_{x}(\mathbf{0})<0$, depending on the sign of $B$ and the first Lyapunov coefficient $\ell_{1}$.

In the sequel, we present examples which exhibit the behaviors stated in Theorems 4.7 and 4.8 
and in Proposition 4.11. First, consider $Z^{\alpha}$ given by

$$
Z_{\alpha}(x, y)=\left\{\begin{array}{l}
X_{\alpha}(x, y)=\left(1+2 x, x+\frac{7}{2} y-\alpha\right) \\
Y(x, y)=(-1,-3 x)
\end{array}\right.
$$

which has a visible-invisible fold at the origin for $\alpha=0$ and satisfies $(\operatorname{det} Z)_{x}(\mathbf{0})=-2$.

The $\varphi$-regularization $Z_{\varepsilon}^{\alpha}(x, y)$ in coordinates $(x, v)$ where $y=\varepsilon v$ has the form

$$
Z_{\varepsilon}^{\alpha}(x, \varepsilon v)=\left\{\begin{array}{l}
\dot{x}=2 x+\varphi(v)(2+2 x), \\
\varepsilon \dot{v}=-2 x-\alpha+\frac{7 \varepsilon v}{2}+\varphi(v)\left(4 x-\alpha+\frac{7 \varepsilon v}{2}\right),
\end{array}\right.
$$

Example 4.3 (Supercritical Hopf bifurcation for the visible-invisible fold). Consider the transition $\operatorname{map} \varphi(v)$

$$
\varphi(v)=v^{5}+\frac{3}{2} v^{3}+\frac{1}{2} v, \text { for } v \in(-1,1) .
$$

The point is $P(\alpha, \varepsilon)=\left(-\frac{1}{2} \alpha+\mathcal{O}_{2}(\alpha, \varepsilon), 0+\mathcal{O}_{2}(\alpha, \varepsilon)\right)$ and the bifurcation curves are:

$$
\begin{aligned}
& \mathcal{D}=\left\{(\alpha, \varepsilon): \varepsilon=\frac{9}{32} \alpha^{2}+\mathcal{O}\left(\alpha^{3}\right)\right\} \\
& \mathcal{H}=\left\{(\alpha, \varepsilon): \alpha=\frac{11}{3} \varepsilon+\mathcal{O}\left(\varepsilon^{2}\right)\right\} .
\end{aligned}
$$

The first Lyapunov coefficient is $\ell_{1}(\alpha(\varepsilon), \varepsilon)=\frac{1}{\sqrt{\varepsilon}}(-23.15+\mathcal{O}(\varepsilon))$, therefore the Hopf bifurcation is supercritical. The Canard trajectory occurs over the curve

$$
\mathcal{C}=\left\{(\alpha, \varepsilon): \alpha=1.98 \varepsilon+\mathcal{O}\left(\varepsilon^{3 / 2}\right)\right\}
$$

therefore, the stated in Theorem 4.7 holds.

We fix $\varepsilon=0.01$ and vary $\alpha$ sufficiently small. We obtain $\alpha_{\mathcal{D}}^{ \pm}\left(\varepsilon_{0}\right) \approx \pm 0.18, \alpha_{\mathcal{H}}\left(\varepsilon_{0}\right) \approx 0.03$ and $\alpha_{\mathcal{C}}\left(\varepsilon_{0}\right) \approx 0.019$ obtained by the intersection between the line $\varepsilon=\varepsilon_{0}$ and curves $\mathcal{D}, \mathcal{H}$ and $\mathcal{C}$ respectively.

In Fig. 29(a) $\alpha=0.015$ : the stable Fenichel manifold $\Lambda_{\varepsilon}^{\alpha, s}$ is above the unstable Fenichel manifold $\Lambda_{\varepsilon}^{\alpha, u}$ and the critical point $P(\alpha, \varepsilon)$ is an unstable focus. In Fig. 29(b) $\alpha=0.019$ : the Fenichel manifolds $\Lambda_{\varepsilon}^{\alpha, u}$ and $\Lambda_{\varepsilon}^{\alpha, s}$ are becoming closer. In Fig. 29(c), $\alpha=0.02$ : the Canard has occurred and a big stable orbit $\Delta_{\varepsilon}^{\alpha, \mathcal{C}}=\Delta_{\varepsilon}^{\alpha, s}$ appears. In Figures 29(c) to 29(e), one can see that the amplitude of the stable periodic orbit decreases while $\alpha$ approaches the value $\alpha_{\mathcal{H}}$.

In Fig. 29(f) $\alpha=0.05$ : the subcritical Hopf bifurcation has occurred and the stable periodic orbit no longer exists. The critical point $P(\alpha, \varepsilon)$ is an stable focus and there are no periodic orbits. In Figure 31(a) we show the behavior of the Melnikov function for this example.

Example 4.4 (Subcritical Hopf bifurcation for the visible-invisible fold). Consider the cubic transition map (4.5). The critical point is $P(\alpha, \varepsilon)=\left(-\frac{1}{2} \alpha+\mathcal{O}_{2}(\alpha, \varepsilon), \mathcal{O}_{2}(\alpha, \varepsilon)\right)$, and the bifurcation curves $\mathcal{D}$, and $\mathcal{H}$ are given by:

$$
\begin{aligned}
& \mathcal{D}=\left\{(\alpha, \varepsilon): \varepsilon=0.84 \alpha^{2}+\mathcal{O}\left(\alpha^{3}\right)\right\} \\
& \mathcal{H}=\left\{(\alpha, \varepsilon): \alpha=1.22 \varepsilon+\mathcal{O}\left(\varepsilon^{2}\right)\right\} .
\end{aligned}
$$

The first Lyapunov coefficient is $\ell_{1}(\alpha(\varepsilon), \varepsilon)=\frac{1}{\sqrt{\varepsilon}}(0.57+\mathcal{O}(\varepsilon))$, therefore the Hopf bifurcation is subcritical. The Canard trajectory occurs over the curve

$$
\mathcal{C}=\left\{(\alpha, \varepsilon): \alpha=1.21 \varepsilon+\mathcal{O}\left(\varepsilon^{3 / 2}\right)\right\}
$$

therefore, the stated in Theorem 4.8 holds. 


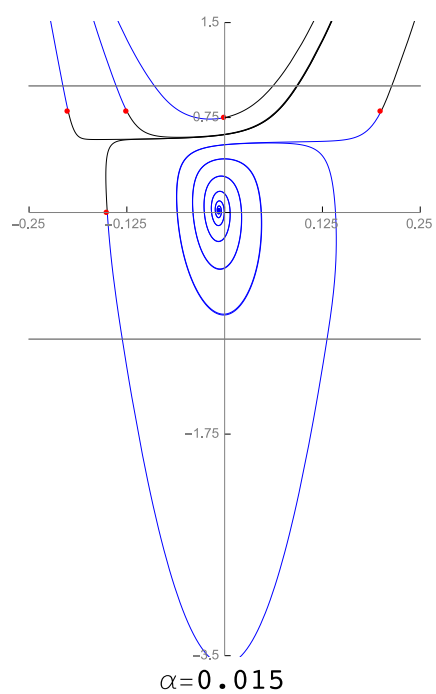

(a)

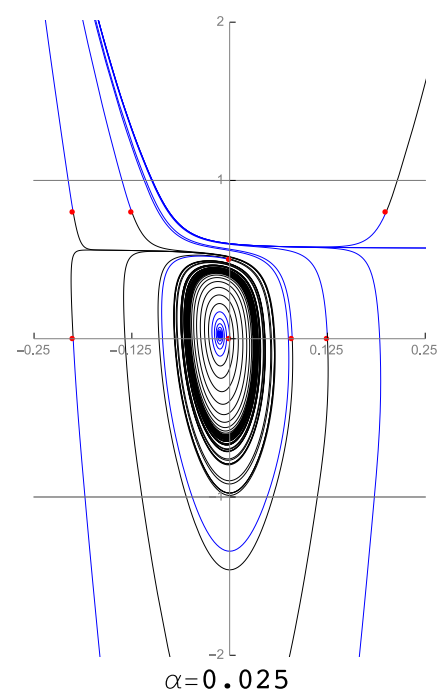

(d)

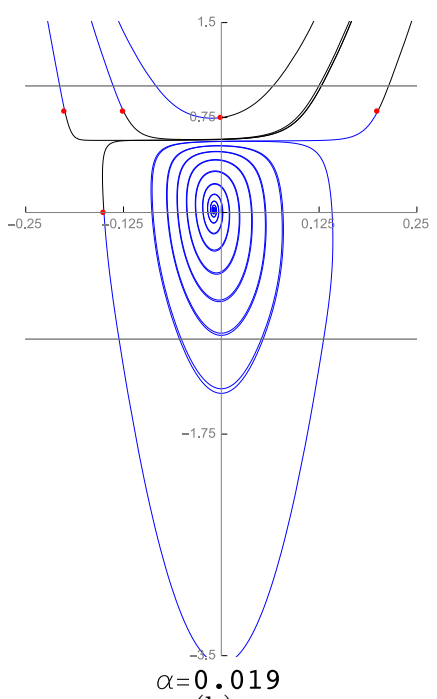

(b)

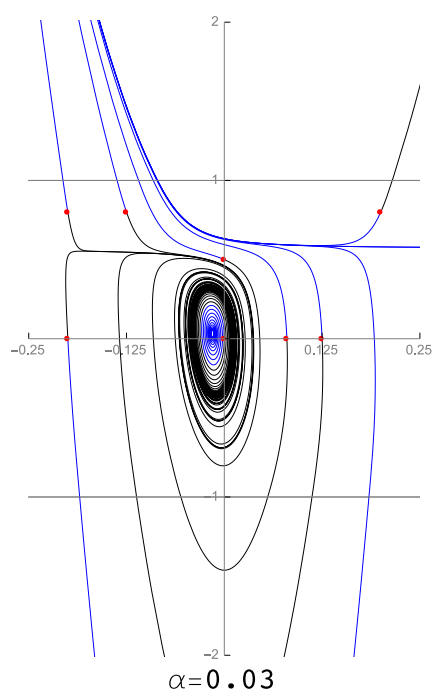

(e)

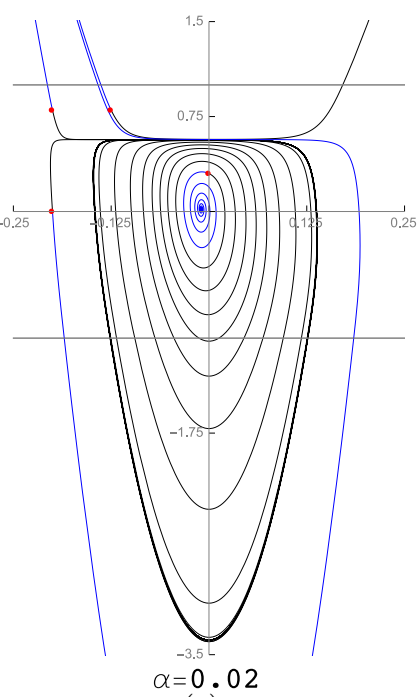

(c)

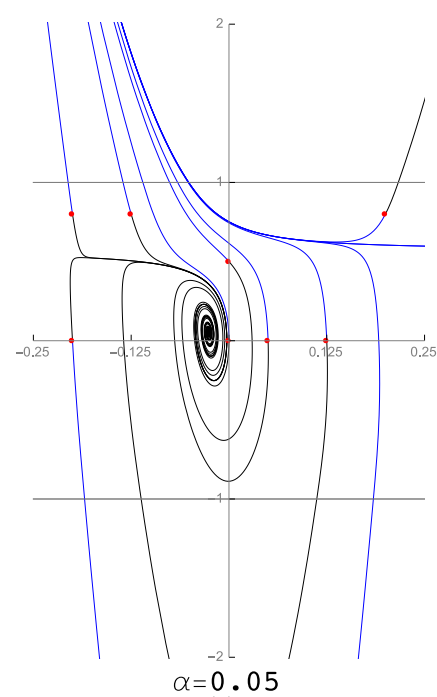

(f)

Initial condition

Negative time

Positive Time

Figure 29: Example 4.3 Trajectories for the regularized vector field $Z_{\varepsilon}^{\alpha}$ for different values of $\alpha$ and $\varepsilon=0.01$.

Fix $\varepsilon_{0}=0.01$ and consider values of $\alpha_{\mathcal{D}}^{ \pm}\left(\varepsilon_{0}\right) \approx \pm 0.1, \alpha_{\mathcal{H}}\left(\varepsilon_{0}\right) \approx 0.01222$ and $\alpha_{\mathcal{C}}\left(\varepsilon_{0}\right) \approx 0.01215$ obtained by the intersection between the line $\varepsilon=\varepsilon_{0}$ and curves $\mathcal{D}, \mathcal{H}$ and $\mathcal{C}$, respectively. We are going to focus our attention to the region inside the parabola $\mathcal{D}$.

In Figures 30(a) to 30(f) one can see the evolution of the dynamics of $Z_{\varepsilon}^{\alpha}$ while we vary the parameter $\alpha$. In Fig. 30(a) $\alpha=0.01, \alpha<\alpha_{\mathcal{C}}\left(\varepsilon_{0}\right)$ : the stable Fenichel manifold is above the unstable one and the critical point is an unstable focus. In Fig. 30(b), $\alpha=0.01216$ : the Canard already happened. There exist a big stable periodic orbit $\Delta_{\varepsilon}^{\alpha, s}$ and an unstable focus. In Fig. 30(c), $\alpha=0.0123$ : the subcritical Hopf bifurcation has occurred and the critical point $P(\alpha, \varepsilon)$ is a stable focus. An small unstable periodic $\Delta_{\varepsilon}^{\alpha, u}$ appears. In Figures $30(\mathrm{c})$ and $30(\mathrm{e})$, the two periodic orbits $\Delta_{\varepsilon}^{\alpha, u}, \Delta_{\varepsilon}^{\alpha, s}$ coexist until the parameter $\alpha$ reaches the value $\alpha_{\mathcal{S}}\left(\varepsilon_{0}\right)$. In Fig. 30(f) $\alpha=0.0127$ : there are no periodic orbits and the stable focus $P(\alpha, \varepsilon)$ is global stable. This means that the value $\alpha_{\mathcal{S}}\left(\varepsilon_{0}\right)$ given by the intersection between the curve $\mathcal{S}$ and the line $\varepsilon=0.01$ belongs to $I_{\mathcal{S}}=(0.01216,0.01217)$. Therefore, for $\alpha>\alpha_{\mathcal{S}}$ we have only an stable focus and no periodic orbits. In Figure 31(b) we show the behavior of the Melnikov function for this example.

Remark 4.13. We want to emphasize that, due to theorem 4.10, the hypothesis of Theorem 4.8 can not be fulfilled if we use a linear regularization function $\varphi$. Therefore, the importance of Example 4.4 


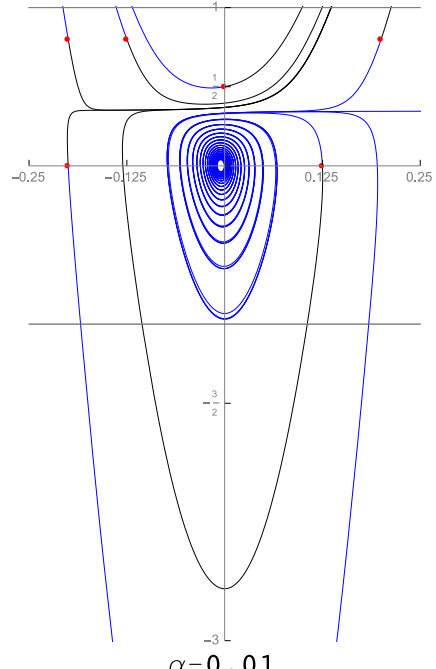

$=0.01$

(a)

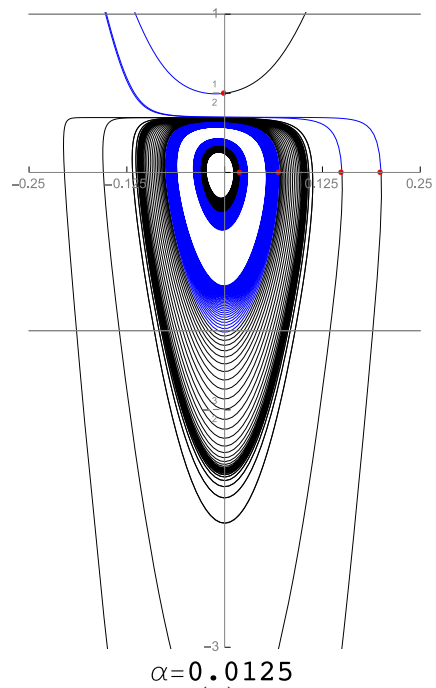

(d)

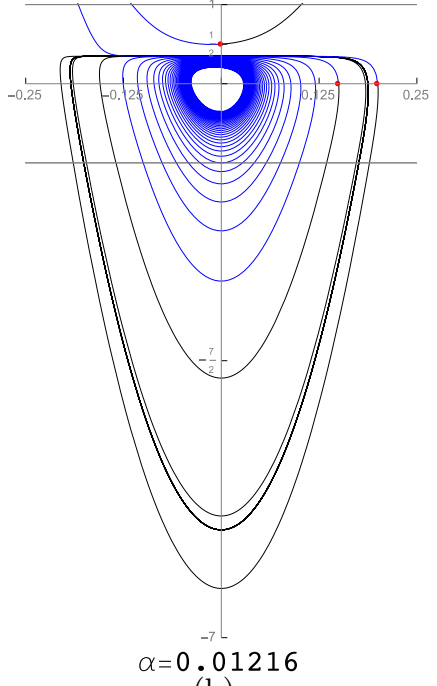

(b)

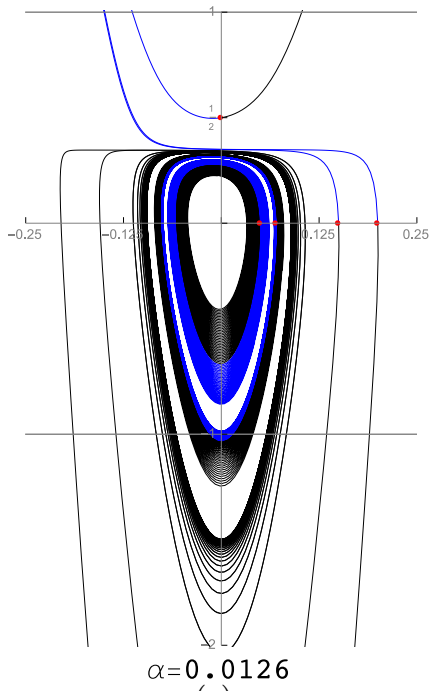

(e)

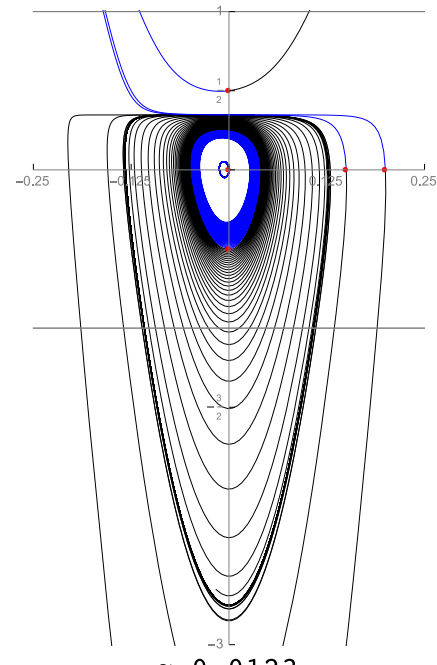

$\alpha=0.0123$

(c)

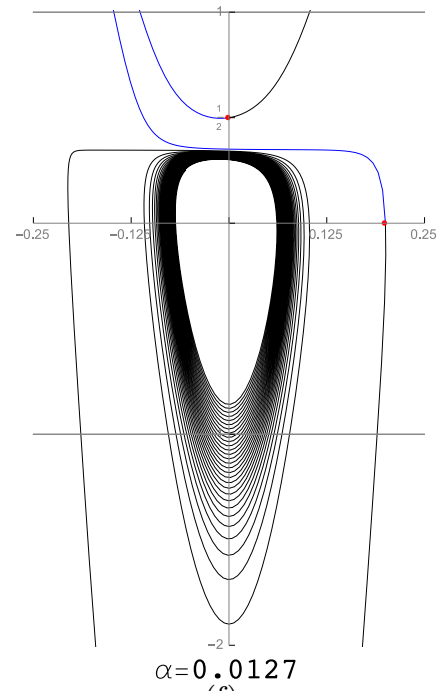

(f)

Initial condition

Negative time

Positive Time

Figure 30: Some trajectories of the regularized system $Z_{\varepsilon}^{\alpha}$ of Example 4.4 for different values of the parameter $\alpha$ and $\varepsilon=0.01$.

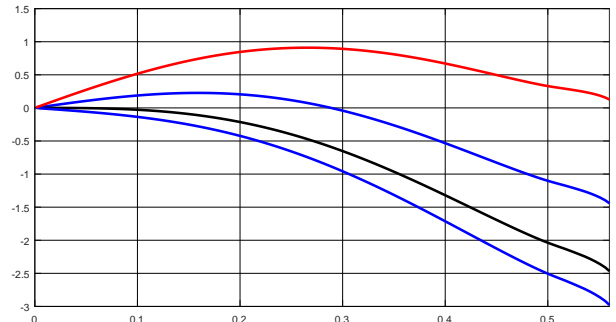

(a)

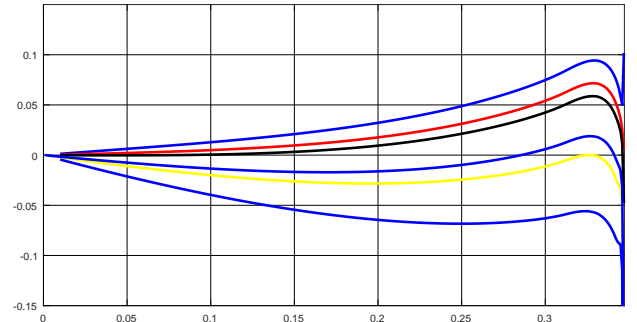

(b)

Figure 31: (a) The Melnikov function for example 4.3 $\delta=\delta_{\mathcal{H}}$ in black is supercritical, $\delta=\delta_{\mathcal{C}}$ in red. For $\delta_{\mathcal{C}} \leq \delta \leq \delta_{\mathcal{H}}$, $M(v, \delta)$ has one zero with negative slope. (b) The Melnikov function for Example $4.4 \delta=\delta_{\mathcal{H}}$ in black is subcritical, $\delta=\delta_{\mathcal{C}}$ in red, $\delta=\delta_{\mathcal{S}}$ in yellow. $M(v, \delta)$ has one zero with negative slope for $\delta_{\mathcal{H}} \leq \delta=\delta_{\mathcal{H}}$, two zeros for $\delta_{\mathcal{H}} \leq \delta \leq \delta_{\mathcal{S}}$ and no zeros for $\delta>\delta_{\mathcal{S}}$ and $\delta<\delta_{\mathcal{C}}$ 
is to show that the hypothesis of Theorem 4.8 are achievable for a $\mathcal{C}^{2}$ transition map.

In what follows, we provide an example to demonstrate that the sign of the coefficient $B$ depends directly of the transition function $\varphi$. For examples 4.5 and 4.6 . consider the vector field

$$
Z_{\alpha}(x, y)=\left\{\begin{array}{l}
X_{\alpha}=\left(1+0.2 x,-\alpha+x\left(8 x^{2}+3 x+1\right)-4 y\right) \\
Y(x, y)=\left(-1,-x\left(8 x^{2}+3 x+3\right)\right)
\end{array}\right.
$$

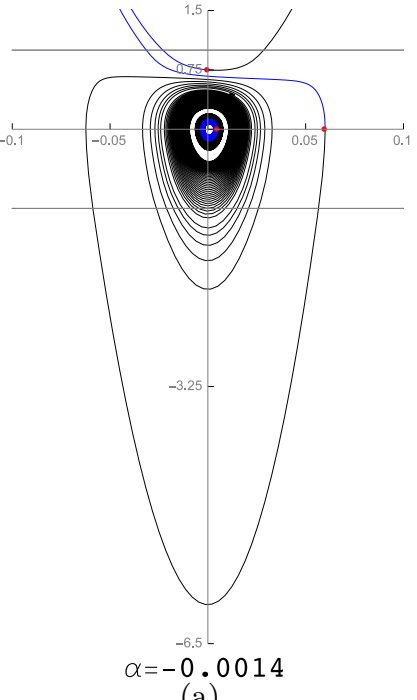

(a)

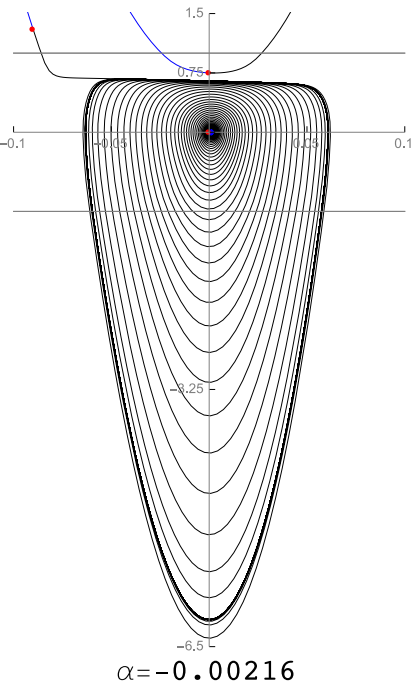

(b)

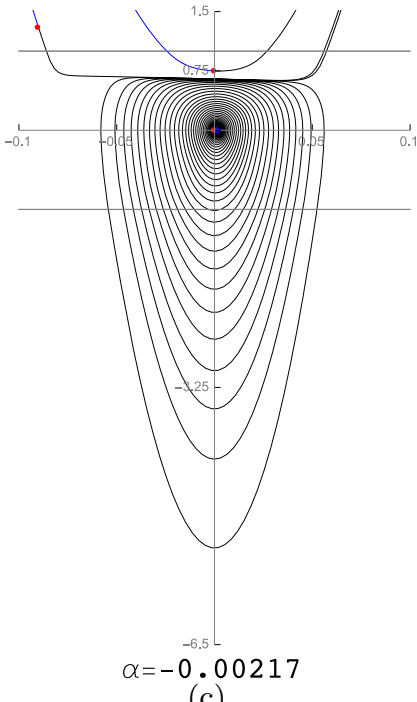

(c)

Initial condition

Negative time

Positive Time

Figure 32: Some trajectories of the regularized system $Z_{\varepsilon}^{\alpha}$ of Example 4.5 for different values of the parameter $\alpha$ and $\varepsilon=0.001$.

Example 4.5 (A stable periodic orbit near the Canard: $B<0$ ). Consider the piecewise vector field (4.16) and let

$$
\varphi(v)=-\frac{5 v^{7}}{2}+\frac{9 v^{5}}{2}-2 v^{3}+v, v \in(-1,1) .
$$

The critical point is $P(\alpha, \varepsilon)=\left(-\frac{1}{2} \alpha+\mathcal{O}_{2}(\alpha, \varepsilon), \mathcal{O}_{2}(\alpha, \varepsilon)\right)$ and the bifurcation curves $\mathcal{D}$, and $\mathcal{H}$ are given by:

$$
\begin{aligned}
\mathcal{D} & =\left\{(\alpha, \varepsilon): \varepsilon=0.562 \alpha^{2}+\mathcal{O}\left(\alpha^{3}\right)\right\} \\
\mathcal{H} & =\left\{(\alpha, \varepsilon): \alpha=-1.26 \varepsilon+\mathcal{O}\left(\varepsilon^{2}\right)\right\} .
\end{aligned}
$$

The first Lyapunov coefficient is $\ell_{1}(\alpha(\varepsilon), \varepsilon)=\frac{1}{\sqrt{\varepsilon}}(-6.3+\mathcal{O}(\varepsilon))$, therefore the Hopf bifurcation is supercritical.

The Canard trajectory occurs over the curve

$$
\mathcal{C}=\left\{(\alpha, \varepsilon): \alpha=-2.167 \varepsilon+\mathcal{O}\left(\varepsilon^{3 / 2}\right)\right\} .
$$

The coefficient $B=-2.17<0$ (see $(4.13)$, therefore, the stated in the first item of Proposition 4.11 holds.

Fix $\varepsilon_{0}=0.001$ and consider values of $\alpha_{\mathcal{D}}^{ \pm}\left(\varepsilon_{0}\right) \approx \pm 0.042, \alpha_{\mathcal{H}}\left(\varepsilon_{0}\right) \approx-0.0012$ and $\alpha_{\mathcal{C}}\left(\varepsilon_{0}\right) \approx$ -0.0021 obtained by the intersection between the line $\varepsilon=\varepsilon_{0}$ and curves $\mathcal{D}, \mathcal{H}$ and $\mathcal{C}$, respectively.

We focus our attention in the region close to the canard curve $\mathcal{C}$, in order to show the behavior explained in the first item of Proposition 4.11

In Figure 32 we illustrate some trajectories of the vector field $Z_{\varepsilon}^{\alpha}$ for $\alpha=\delta \varepsilon$ with $\delta$ near the value $\delta_{\mathcal{C}}$. Observe that, for these values of $\alpha$ the critical point $P(\alpha, \varepsilon)$ is always an unstable focus. 


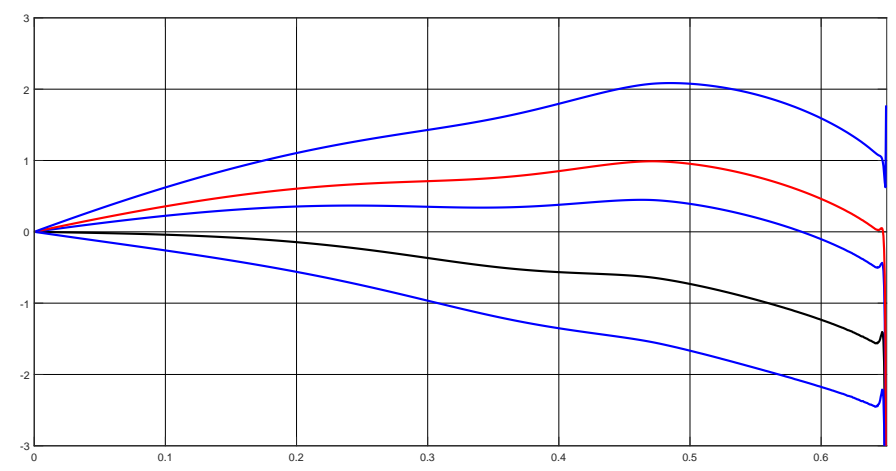

Figure 33: The Melnikov function for example $4.5 \delta=\delta_{\mathcal{H}}$ in black is supercritical, $\delta=\delta_{\mathcal{C}}$ in red. $M(v, \delta)$ has only one zero for $\delta$ near $\delta_{\mathcal{C}}$ and it has negative slope.

In Fig. 32(a), $\alpha=-0.00140, \alpha_{\mathcal{C}} \ll \alpha \leq \alpha_{\mathcal{H}}$ : a stable periodic orbit exists and the stable Fenichel manifold is above the unstable one. In Figure 32(b) $\alpha=-0.00216, \alpha \leq \alpha_{\mathcal{C}}$, one can see a big stable periodic orbit, which corresponds to the periodic orbit $\Delta_{\varepsilon}^{\alpha, C}$. Finally, in Fig. 32(c), $\alpha=-0.00217, \alpha>\alpha_{\mathcal{C}}$ : the Canard already happened and the unstable Fenichel manifold is above the stable one. The periodic orbit $\Delta_{\varepsilon}^{\alpha, C}$ no longer exist. In Figure 33 one can see the Melnikov function for different values of $\delta$ when $\alpha=\delta \varepsilon$.

Example 4.6 (A unstable periodic orbit near the Canard: $B>0$ ). Consider the piecewise vector field (4.16) and the cubic transition map (4.5).

The critical point is $P(\alpha, \varepsilon)=\left(-\frac{1}{2} \alpha+\mathcal{O}_{2}(\alpha, \varepsilon), \mathcal{O}_{2}(\alpha, \varepsilon)\right)$ and the bifurcation curves $\mathcal{D}$, and $\mathcal{H}$ are given by:

$$
\begin{aligned}
\mathcal{D} & =\left\{(\alpha, \varepsilon): \varepsilon=0.8437 \alpha^{2}+\mathcal{O}\left(\alpha^{3}\right)\right\} \\
\mathcal{H} & =\left\{(\alpha, \varepsilon): \alpha=-0.8444 \varepsilon+\mathcal{O}\left(\varepsilon^{2}\right)\right\} .
\end{aligned}
$$

The first Lyapunov coefficient is $\ell_{1}(\alpha(\varepsilon), \varepsilon)=\frac{1}{\sqrt{\varepsilon}}(-1.09+\mathcal{O}(\varepsilon))$, therefore the Hopf bifurcation is supercritical.

The Canard trajectory occurs over the curve

$$
\mathcal{C}=\left\{(\alpha, \varepsilon): \alpha=-1.01013 \varepsilon+\mathcal{O}\left(\varepsilon^{3 / 2}\right)\right\} .
$$

The coefficient $B=5.66>0$ (see(4.13)], therefore, the stated in the second item of Proposition 4.11 holds. The system $Z_{\varepsilon}^{\alpha}$ has a saddle-node bifurcation of periodic orbits in some curve $\mathcal{\mathcal { S }}$ located to the left of the canard curve $\mathcal{C}$.

Fix $\varepsilon_{0}=0.001$ and consider values of $\alpha_{\mathcal{D}}^{ \pm}\left(\varepsilon_{0}\right) \approx \pm 0.1, \alpha_{\mathcal{H}}\left(\varepsilon_{0}\right) \approx-0.0084$ and $\alpha_{\mathcal{C}}\left(\varepsilon_{0}\right) \approx$ -0.0101013 obtained by the intersection between the line $\varepsilon=\varepsilon_{0}$ and curves $\mathcal{D}, \mathcal{H}$ and $\mathcal{C}$, respectively.

Once again, we focus our attention in the region close to the canard curve $\mathcal{C}$, in order to show the behavior explained in Proposition 4.11.

In Figures 34(a) to 34(d) we illustrate some trajectories of the vector field $Z_{\varepsilon}^{\alpha}$ for $\alpha=\delta \varepsilon$ with $\delta$ near the value $\delta_{\mathcal{C}}$. Observe that, for these values of $\alpha$ the critical point $P(\alpha, \varepsilon)$ is always an unstable focus. We vary the parameter values from right to left on the bifurcation diagram, that is, from the Hopf bifurcation to the Canard direction.

In Fig. 34(a) $\alpha=-0.001, \alpha_{\mathcal{C}}<\alpha<\alpha_{\mathcal{H}}$ : a small stable periodic orbit exists and the unstable Fenichel manifold is bellow the stable one. In Fig. 34(b) $\alpha=-0.00101014, \alpha_{\tilde{\mathcal{S}}}<\alpha \lesssim \alpha_{\mathcal{C}}$ : the stable Fenichel manifold is above the unstable one. For this value of $\alpha$, one can see two periodic orbits: a bigger one which is unstable and corresponds to the periodic orbit $\Delta_{\varepsilon}^{\alpha, C}$ and an smaller one, which is stable and correspond to the periodic orbit $\Delta_{\varepsilon}^{\alpha, s}$. 


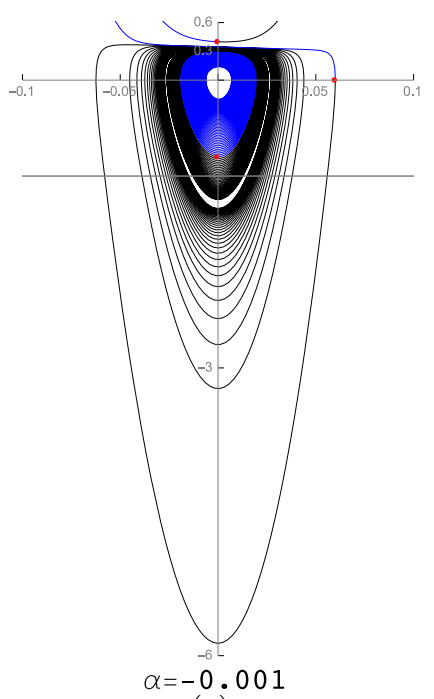

(a)

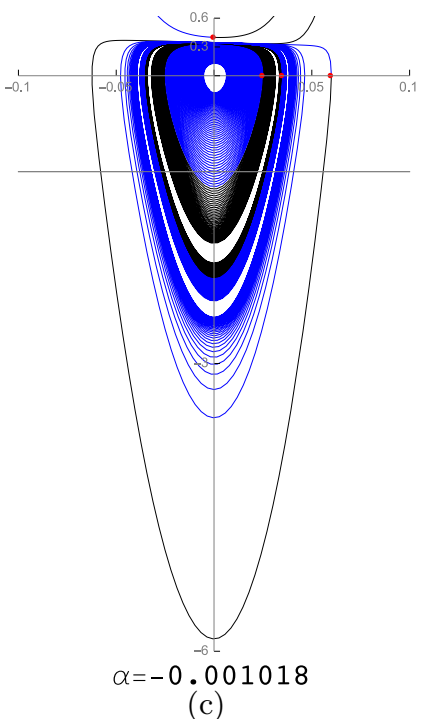

(c)

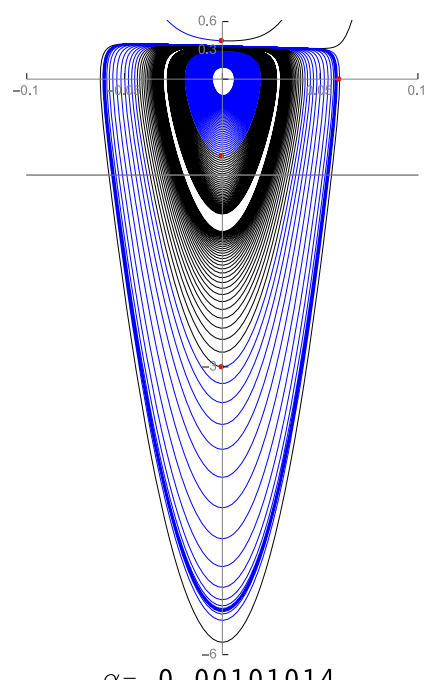

(b)

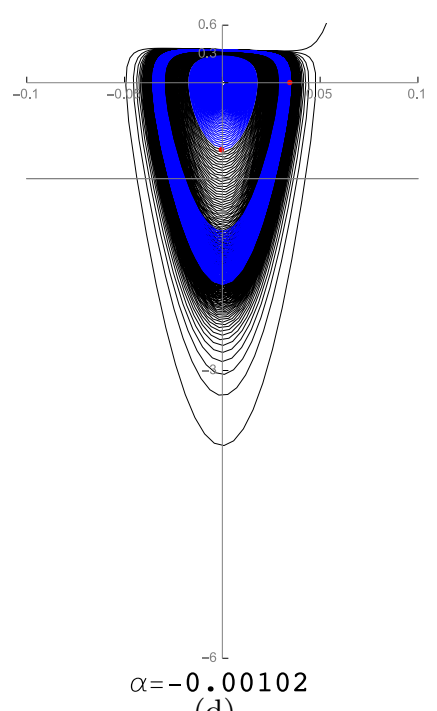

(d)

Initial condition

Negative time

Positive Time

Figure 34: Some trajectories of the regularized system $Z_{\varepsilon}^{\alpha}$ of Example 4.6 for different values of the parameter $\alpha$ and $\varepsilon=0.001$.

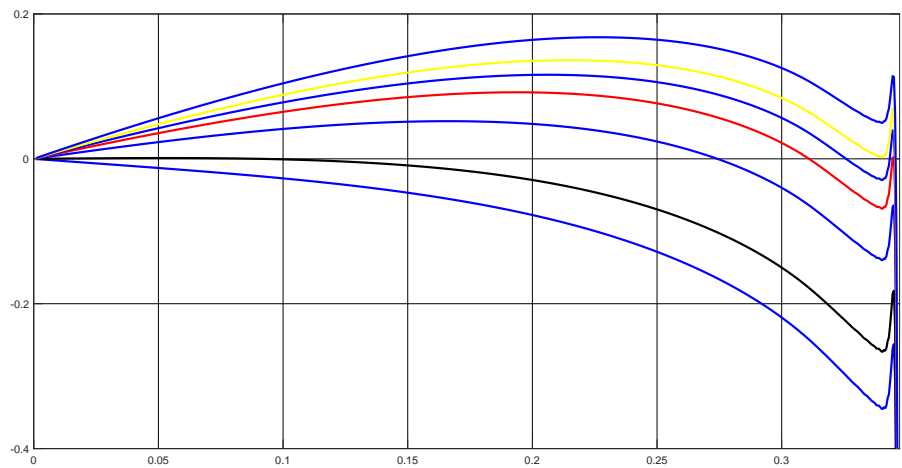

Figure 35: The Melnikov function for example 4.6 $\delta=\delta_{\mathcal{H}}$ in black is subcritical, $\delta=\delta_{\mathcal{C}}$ in red, $\delta=\delta_{\tilde{\mathcal{S}}}$ in yellow. $M(v, \delta)$ has no zeros for $\delta<\delta_{\tilde{\mathcal{S}}}$, two zeros for $\delta_{\tilde{\mathcal{S}}} \leq \delta \leq \delta_{\mathcal{C}}$, one zero with negative slope for $\delta_{\mathcal{C}}<\delta<\delta_{\mathcal{H}}$ and no zeros for $\delta>\delta_{\mathcal{H}}$.

Since for $\alpha=-0.001018$ we have two periodic orbits and for $\alpha=-0.0102$ we have no periodic orbits (see figures 34(c) and Fig. 34(d) it follows that the value of $\alpha_{\tilde{\mathcal{S}}}\left(\varepsilon_{0}\right)$ given by Proposition 4.11 
belongs to $I_{\tilde{\mathcal{S}}}=(-0.0102,-0.01018)$. Moreover, in these pictures, we see that the size of the smaller stable periodic orbit increases while the size of the big unstable periodic orbit decreases. In Figure 35 one can see the Melnikov function for different values of $\delta$ when $\alpha=\delta \varepsilon$.

\subsubsection{The saddle case: $(\operatorname{det} Z)_{x}(0)>0$}

When $(\operatorname{det} Z)_{x}(\mathbf{0})>0$, for any $\alpha$ and $\varepsilon>0$ sufficiently small, by Lemma 3.3 and Proposition 3.7 the regularized vector field $Z_{\varepsilon}^{\alpha}$ has a critical point $P(\alpha, \varepsilon)$ which is a saddle.

Analogously to the visible-visible case, although the point $P(\alpha, \varepsilon)$ maintains the same character for all $\alpha, \varepsilon$, a "bifurcation" on the Fenichel manifolds when $\alpha$ crosses the $\alpha=0$ value occurs.

Using the results about the critical manifolds in subsubsection 3.2.2, for each fixed $\alpha \neq 0$ and any compact set of the critical manifolds $\Lambda_{0}^{\alpha, s}$ and $\Lambda_{0}^{\alpha, u}$ excluding the tangency points $\left(T_{X}^{\alpha}, 1\right)$ and $\left(T_{Y}^{\alpha},-1\right)$, for $0<\varepsilon<\varepsilon_{0}(\alpha)$, there exist two normally hyperbolic invariant manifold $\Lambda_{\varepsilon}^{\alpha, s}$ and $\Lambda_{\varepsilon}^{\alpha, u}$ which are $\varepsilon$-close to $\Lambda_{0}^{\alpha, s}$ and $\Lambda_{0}^{\alpha, u}$, respectively (see Fig. 14(a) . Moreover,

- for $\alpha<0, \Lambda_{\varepsilon}^{\alpha, s}$ is the unstable manifold of the saddle point $P(\alpha, \varepsilon)$,

- for $\alpha>0, \Lambda_{\varepsilon}^{\alpha, u}$ is the stable manifold of the saddle point $P(\alpha, \varepsilon)$.

Observe that for $x<T_{X}^{\alpha, \varepsilon}$ the vector $X^{\alpha}(x, 1)$ points inward to the regularization zone and points outwards to the regularization zone for $x>T_{X}^{\alpha, \varepsilon}$. Analogously, for $x<T_{Y}^{\alpha, \varepsilon}$ the vector $Y^{\alpha}(x,-1)$ points inwards to the regularization zone for $x<T_{Y}^{\alpha, \varepsilon}$ and outwards to the regularization zone for $x>T_{Y}^{\alpha, \varepsilon}$.
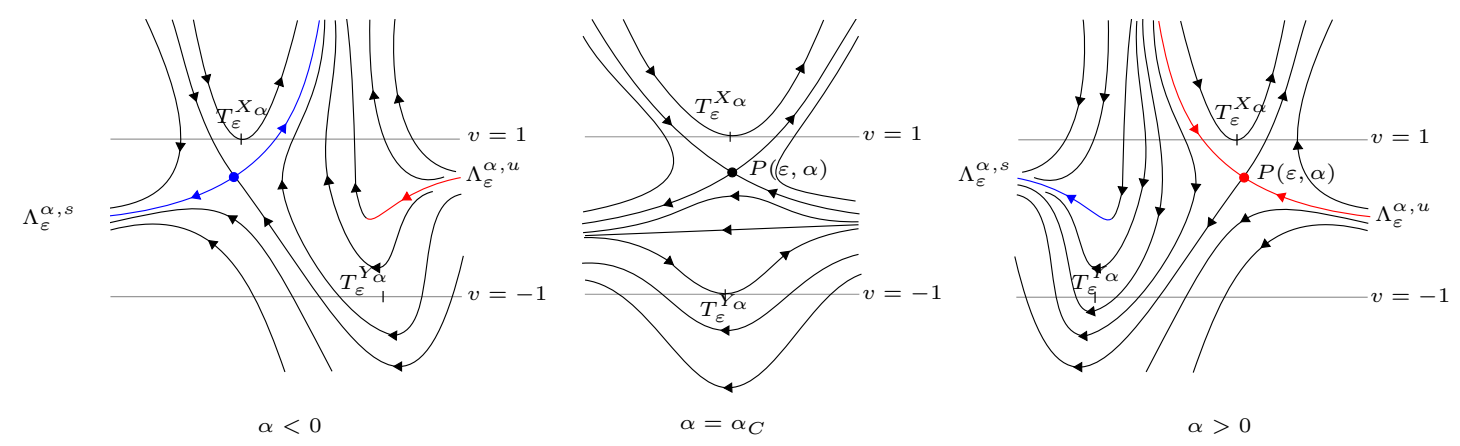

Figure 36: The phase portrait of $Z_{\varepsilon}^{\alpha}$ for $\varepsilon>0$ and different $\alpha$.

The above information and the fact that the dynamics over the Fenichel Manifold $\Lambda_{\varepsilon}^{\alpha}$ is the same of the critical manifold $\Lambda_{0}^{\alpha}$, it follows that

- for $\alpha<0$, the stable Fenichel manifold $\Lambda_{\varepsilon}^{\alpha, s}$ intersects the section $\{v=1\}$ on the right of the tangency point $T_{X}^{\alpha, \varepsilon}$. The unstable Fenichel manifold $\Lambda_{\varepsilon}^{\alpha, u}$ can intersect or not the section $v=-1$, if this intersection occurs it is located to the right of the tangency point $T_{Y}^{\alpha, \varepsilon}$,

- for $\alpha>0$, the unstable Fenichel manifold $\Lambda_{\varepsilon}^{\alpha, u}$ intersects the section $\{v=1\}$ on the left of the tangency point $T_{X}^{\alpha, \varepsilon}$. The stable Fenichel manifold $\Lambda_{\varepsilon}^{\alpha, s}$ can intersect or not the section $v=-1$, if this intersection occurs it is located to the left of the tangency point $T_{Y}^{\alpha, \varepsilon}$.

The phase portrait of $Z_{\varepsilon}^{\alpha}$ for $\alpha \neq 0$ is given in Figure 36 .

Remark 4.14. Observe that even if the Canard trajectory also exists when $(\operatorname{det} Z)_{x}(\mathbf{0})>0$ it does not play a special role in this case. However, it is illustrated in Figure 36.

\section{Melnikov Method}

This section will be devoted to study the existence and bifurcations of periodic orbits when $(\operatorname{det} Z)_{x}(\mathbf{0})<0$ and therefore the fixed point is a focus, using the Melnikov method. We will prove 
Proposition 5.1 and later, in subsection 7.1 we will proof Proposition 4.5, using the results in this section. In fact, one could easily recover the results of Theorems 4.3, 4.4 for the invisible-invisible case, and give the proof of Proposition 4.5 and also Theorems 4.7 and 4.8 for the visible-invisible case using classical perturbation theory, also known as Melnikov theory. We will see that the Melnikov function contains all the information about the periodic orbits of the system while their size in the $x$-direction is "small" respect to the parameter $\varepsilon$.

The first observation is that examples $4.1,4.2,4.3,4.4,4.5$ and 4.6 show that the periodic orbit which arises in the Hopf bifurcation and the possible saddle-node bifurcations of the system occur in a region of the phase space where $x=O(\sqrt{\varepsilon})$.

To analyze the system in this region when $\alpha=\delta \varepsilon$ we perform the change $u=\sqrt{\varepsilon} x$ in system (3.5) and a suitable scaling of time $t=\sqrt{\varepsilon} \tau$. Calling $\gamma=\sqrt{\varepsilon}$ the system reads:

$$
\begin{aligned}
& \frac{d u}{d \tau}=F^{1}(0, v ; 0,0)+\gamma F_{x}^{1}(0, v ; 0,0) u+O\left(\gamma^{2}\right) \\
& \frac{d v}{d \tau}=F_{x}^{2}(0, v ; 0,0) u+\gamma\left(\frac{1}{2} F_{x x}^{2}(0, v ; 0,0) u^{2}+F_{\varepsilon}^{2}(0, v ; 0,0)+\delta \tilde{F}^{2}(0, v ; 0,0)\right)+\mathcal{O}\left(\gamma^{2}\right)
\end{aligned}
$$

Where, fixing $M>0$, the $\mathcal{O}\left(\gamma^{2}\right)$ terms are bounded by $K \gamma^{2}$ for $|u| \leq M$, and $|\gamma v| \leq M$, and $K=K(M)$.

The main observation is that for $\gamma=0$ the system is integrable and the function

$$
H(u, v)=\frac{u^{2}}{2}+V(v), \quad V(v)=-\int_{v^{*}}^{v} \frac{F^{1}(0, r ; 0,0)}{F_{x}^{2}(0, r ; 0,0)} d r,
$$

where $\left(0, v^{*}\right)$ is a critical point and $v^{*}$ is given in $(3.12)$, is a first integral of the system.

As $(\operatorname{det} Z)_{x}(\mathbf{0})<0$, one has that $V\left(v^{*}\right)=0, V^{\prime}\left(v^{*}\right)=\frac{F^{1}\left(0, v^{*} ; 0,0\right)}{F_{x}^{2}\left(0, v^{*} ; 0,0\right)}=0$ and

$$
V^{\prime \prime}\left(v^{*}\right)=-\frac{\varphi^{\prime}\left(v^{*}\right)\left(\left(X^{1}-Y^{1}\right)(\mathbf{0})\right)^{2}}{2(\operatorname{det} Z)_{x}(\mathbf{0})}>0
$$

Therefore the point $\left(0, v^{*}\right)$ is a non-linear center surrounded by periodic solutions. We want to check which of these solutions persists when $\gamma \neq 0$ small enough, depending of the value of $\delta$. For Hamiltonian systems, one can apply the classical Melnikov method to show that, generically, fixing $\delta$, one periodic orbit can persist after the perturbation if some open condition is satisfied. For the system at hand, even if it is not Hamiltonian, it is autonomous and therefore one can change time to get a Hamiltonian system and then apply the classical theory for the Hamiltonian case (see, for instance [HG83; Wig03, [Tes12]).

Nevertheless, to make the paper more self contained, we will do this simple computation here without changes of time. Consider the section

$$
\Theta=\left\{(0, v), v \geq v^{*}\right\}
$$

and the Poincaré return map $\pi: \Theta \rightarrow \Theta$, given by $\pi\left(0, v_{0}\right)=\left(0, v_{1}\right)$ where $v_{1}=v(T)$ and $T=T\left(v_{0}\right)$ is the time such that the orbit $(u(t), v(t))$ with initial condition $(u(0), v(0))=\left(0, v_{0}\right)$, satisfies $u(T)=0$ and $v(T) \geq v^{*}$. Our goal is to give an asymptotic formula for $v_{1}-v_{0}$. The main observation is that we know a priori that $v_{1}-v_{0}=O(\gamma)$, because for $\gamma=0$ all the orbits are periodic. Therefore, we observe that:

$$
H\left(0, v_{1}\right)-H\left(0, v_{0}\right)=V\left(v_{1}\right)-V\left(v_{0}\right)=V^{\prime}\left(v_{0}\right)\left(v_{1}-v_{0}\right)+O\left(\gamma^{2}\right)
$$

and consequently:

$$
v_{1}-v_{0}=\frac{H\left(0, v_{1}\right)-H\left(0, v_{0}\right)}{V^{\prime}\left(v_{0}\right)}+O\left(\gamma^{2}\right)
$$

Using that $H(u, v)$ is a first integral of system (5.1) for $\gamma=0$ and the Fundamental Theorem of Calculus applied to the function $f(t)=H(u(t), v(t))$, we have that

$$
H\left(0, v_{1}\right)-H\left(0, v_{0}\right)=\int_{0}^{T} \frac{\partial H}{\partial u} \frac{d u}{d t}+\frac{\partial H}{\partial v} \frac{d v}{d t}(u(t), v(t)) d t
$$




$$
\begin{aligned}
& =\gamma \int_{0}^{T}\left(F_{x}^{1}(0, v(t) ; 0,0) u(t)^{2}+V^{\prime}(v(t))\left[\frac{1}{2} F_{x x}^{2}(0, v(t) ; 0,0) u(t)^{2}\right.\right. \\
& \left.\left.+\quad F_{\varepsilon}^{2}(0, v(t) ; 0,0)+\delta \tilde{F}^{2}(0, v(t) ; 0,0)\right]\right) d t+O\left(\gamma^{2}\right) .
\end{aligned}
$$

If we take the initial value $v_{0} \in\left[v^{*}, V\right]$ for any fixed $V>v^{*}$ in invisible-invisible case or $V<\bar{v}$ in the visible-invisible case, one can ensure that, if $\gamma$ is small enough, the solution $u(t)=u_{p}(t)+\mathcal{O}(\gamma)$, $v(t)=v_{p}(t)+\mathcal{O}(\gamma), T=T_{0}+\mathcal{O}(\gamma)$, where $\left(u_{p}(t), v_{p}(t)\right)$ is the periodic solution of the unperturbed system with initial condition $\left(0, v_{0}\right)$ and $T_{0}=T_{0}\left(v_{0}\right)$ its period. Then, using $(5.2)$ we obtain:

$$
v_{1}-v_{0}=\gamma M\left(v_{0}, \delta\right)+\mathcal{O}\left(\gamma^{2}\right)
$$

where

$$
\begin{aligned}
M\left(v_{0}, \delta\right) & =\frac{1}{V^{\prime}\left(v_{0}\right)} \int_{0}^{T_{0}}\left(F_{x}^{1}\left(0, v_{p}(t) ; 0,0\right) u_{p}(t)^{2}+V^{\prime}\left(v_{p}(t)\right)\left[\frac{1}{2} F_{x x}^{2}\left(0, v_{p}(t) ; 0,0\right) u_{p}(t)^{2}\right.\right. \\
& \left.\left.+F_{\varepsilon}^{2}\left(0, v_{p}(t) ; 0,0\right)+\delta \tilde{F}^{2}\left(0, v_{p}(t) ; 0,0\right)\right]\right) d t
\end{aligned}
$$

The function $M\left(v_{0}, \delta\right)$ is known as the Melnikov function. To simplify its expression let's $0 \leq \bar{T}_{0} \leq T_{0}$ be the time where the orbit $\left(u_{p}(t), v_{p}(t)\right)$ intersects $u=0$ for the first time at a point $\left(0, \bar{v}_{0}\right)$, with $\bar{v}_{0}<v^{*}$. Splitting the integral from 0 to $T_{0}$ into two integrals from 0 to $\bar{T}_{0}$ and from $\bar{T}_{0}$ to $T_{0}$, and changing variables in both integrals to $v=v_{p}(t)$, and using that $H\left(u_{p}(t), v_{p}(t)\right)=H\left(0, v_{0}\right)=V\left(v_{0}\right)$, one obtains:

$$
\begin{aligned}
M\left(v_{0}, \delta\right) & =\frac{-2}{V^{\prime}\left(v_{0}\right)} \int_{\bar{v}_{0}}^{v_{0}}\left\{2 F_{x}^{1}(0, v ; 0,0)\left(V\left(v_{0}\right)-V(v)\right)+V^{\prime}(v)\left(F_{x x}^{2}(0 . v ; 0,0)\left(V\left(v_{0}\right)-V(v)\right)\right.\right. \\
& \left.\left.+F_{\varepsilon}^{2}(0, v ; 0,0)+\delta \tilde{F}^{2}(0, v ; 0,0)\right)\right\} \frac{d v}{F_{x}^{2}(0, v ; 0,0) \sqrt{2\left(V\left(v_{0}\right)-V(v)\right)}}
\end{aligned}
$$

where $\bar{v}_{0}$ satisfies $V\left(v_{0}\right)=V\left(\bar{v}_{0}\right)$. Now, integrating by parts the second term in the integral:

$$
\begin{aligned}
M\left(v_{0}, \delta\right) & =\frac{-2}{V^{\prime}\left(v_{0}\right)} \int_{\bar{v}_{0}}^{v_{0}}\left[\frac{F_{x}^{1}(0, v ; 0,0)}{F_{x}^{2}(0, v ; 0,0)}+\frac{\partial}{\partial v}\left\{\frac { 1 } { F _ { x } ^ { 2 } ( 0 , v ; 0 , 0 ) } \left(F_{x x}^{2}(0, v ; 0,0)\left(V\left(v_{0}\right)-V(v)\right)\right.\right.\right. \\
& \left.\left.\left.+F_{\varepsilon}^{2}(0, v ; 0,0)+\delta \tilde{F}^{2}(0, v ; 0,0)\right)\right\}\right] \sqrt{2\left(V\left(v_{0}\right)-V(v)\right)} d v \\
& =\frac{-2}{V^{\prime}\left(v_{0}\right)} \int_{\bar{v}_{0}}^{v_{0}} f\left(v, v_{0}, \delta\right) \sqrt{2\left(V\left(v_{0}\right)-V(v)\right)} d v
\end{aligned}
$$

where:

$$
\begin{aligned}
f\left(v, v_{0}, \delta\right)= & =\frac{F_{x}^{1}(0, v ; 0,0)}{F_{x}^{2}(0, v ; 0,0)}+\frac{\partial}{\partial v}\left\{\frac { 1 } { F _ { x } ^ { 2 } ( 0 , v ; 0 , 0 ) } \left(F_{x x}^{2}(0, v ; 0,0)\left(V\left(v_{0}\right)-V(v)\right)\right.\right. \\
& \left.\left.+F_{\varepsilon}^{2}(0, v ; 0,0)+\delta \tilde{F}^{2}(0, v ; 0,0)\right)\right\} .
\end{aligned}
$$

Proposition 5.1. The Melnikov function $M(v, \delta)$ satisfies:

- $M\left(v^{*}, \delta\right)=0, \forall \delta$;

- $\frac{\partial M}{\partial v}\left(v^{*}, \delta_{\mathcal{H}}\right)=0$;

- In the invisible-invisible case $\frac{\partial^{2}}{\partial v \partial \delta} M\left(v^{*}, \delta_{\mathcal{H}}\right)>0$;

- In the visible-invisible case $\frac{\partial^{2}}{\partial v \partial \delta} M\left(v^{*}, \delta_{\mathcal{H}}\right)<0$;

- If $\frac{\partial^{2}}{\partial v^{2}} M\left(v^{*}, \delta_{\mathcal{H}}\right)>0$ the Hopf bifurcation is subcritical (the Lyapunov coefficient $\ell_{1}>0$ ); 
- If $\frac{\partial^{2}}{\partial v^{2}} M\left(v^{*}, \delta_{\mathcal{H}}\right)<0$ the Hopf bifurcation is supercritical (the Lyapunov coefficient $\left.\ell_{1}<0\right)$.

Proof. It is clear that the integral vanishes at $v^{*}$ for any $\delta$ because in this case $\bar{v}_{0}=v_{0}=v^{*}$, but as $V^{\prime}\left(v^{*}\right)=0$ we need more information about the integral at $v^{*}$. For this reason we split the integral between $\bar{v}_{0}$ and $v^{*}$ and between $v^{*}$ and $v_{0}$. In the first integral we perform the change $v=v^{*}+t\left(\bar{v}_{0}-v^{*}\right)$ and in the second one $v=v^{*}+t\left(v_{0}-v^{*}\right)$ obtaining:

$$
\begin{aligned}
& \int_{\bar{v}_{0}}^{v_{0}} f\left(v, v_{0}, \delta\right) \sqrt{2\left(V\left(v_{0}\right)-V(v)\right)} d v \\
= & \int_{\bar{v}_{0}}^{v^{*}} f\left(v, v_{0}, \delta\right) \sqrt{2\left(V\left(v_{0}\right)-V(v)\right)} d v+\int_{\bar{v}^{*}}^{v_{0}} f\left(v, v_{0}, \delta\right) \sqrt{2\left(V\left(v_{0}\right)-V(v)\right)} d v \\
= & -\left(\bar{v}_{0}-v^{*}\right) \int_{0}^{1} f\left(v^{*}+t\left(\bar{v}_{0}-v^{*}\right), v_{0}, \delta\right) \sqrt{2\left(V\left(v_{0}\right)-V\left(v^{*}+t\left(\bar{v}_{0}-v^{*}\right)\right)\right.} d t \\
+ & \left(v_{0}-v^{*}\right) \int_{0}^{1} f\left(v^{*}+t\left(v_{0}-v^{*}\right), v_{0}, \delta\right) \sqrt{2\left(V\left(v_{0}\right)-V\left(v^{*}+t\left(v_{0}-v^{*}\right)\right)\right)} d t=
\end{aligned}
$$

Next step is to use

$$
V(v)=\left(v-v^{*}\right)^{2} \tilde{V}(v), \tilde{V}\left(v^{*}\right)=\frac{V^{\prime \prime}\left(v^{*}\right)}{2}
$$

obtaining:

$$
\begin{aligned}
& =\left(\bar{v}_{0}-v^{*}\right)^{2} \int_{0}^{1} f\left(v^{*}+t\left(\bar{v}_{0}-v^{*}\right), v_{0}, \delta\right) \sqrt{2\left(\tilde{V}\left(\bar{v}_{0}\right)-t^{2} \tilde{V}\left(v^{*}+t\left(\bar{v}_{0}-v^{*}\right)\right)\right.} d t \\
& +\left(v_{0}-v^{*}\right)^{2} \int_{0}^{1} f\left(v^{*}+t\left(v_{0}-v^{*}\right), v_{0}, \delta\right) \sqrt{2\left(\tilde{V}\left(v_{0}\right)-t^{2} \tilde{V}\left(v^{*}+t\left(v_{0}-v^{*}\right)\right)\right)} d t
\end{aligned}
$$

using these computations and that $\bar{v}_{0}-v^{*}=-\left(v_{0}-v^{*}\right)+O\left(v_{0}-v^{*}\right)^{2}$, one obtains:

$$
\begin{aligned}
M\left(v^{*}, \delta\right) & =0, \\
\frac{\partial M}{\partial v}\left(v^{*}, \delta\right) & =-\frac{\pi}{2 \sqrt{V^{\prime \prime}\left(v^{*}\right)}} f\left(v^{*}, v^{*}, \delta\right) .
\end{aligned}
$$

Now, using the expression of $f$ one can see that this derivative vanishes if $\delta=\delta_{H}$ given in (3.16) Observe that, since $v^{*}$ is a zero of $M(\cdot, \delta)$ for any $\delta$ and a critical point of $M\left(\cdot, \delta_{\mathcal{H}}\right)$, the stability of the critical point $\left(0, v^{*}\right)$ is given by the second derivative $\frac{\partial^{2}}{\partial v^{2}} M\left(v^{*}, \delta_{\mathcal{H}}\right)$. More precisely, this value corresponds to the Lyapunov coefficient of the Hopf bifurcation, which is subcritical when $v^{*}$ is a minimum and it is supercritical when it is a maximum of of $M\left(\cdot, \delta_{\mathcal{H}}\right)$.

Moreover, using that the function $f\left(v, v_{0}, \delta\right)$, and therefore the function $M\left(v_{0}, \delta\right)$ are lineal with respect to $\delta$ one easily obtains:

$$
\frac{\partial^{2}}{\partial v \partial \delta} M\left(v^{*}, \delta_{\mathcal{H}}\right)=\frac{\pi \varphi^{\prime}\left(v^{*}\right)}{4\left((\operatorname{det} Z)_{x}(\mathbf{0})\right)^{2} \sqrt{V^{\prime \prime}\left(v^{*}\right)}}\left(X_{x}^{2} \tilde{Y}^{2}-Y_{x}^{2} \tilde{X}^{2}\right)(\mathbf{0})
$$

which is, by Definition 2.3 and (2.19), positive if both folds are invisible and negative if the folds have opposite visibility.

Once we know the basic properties of the function $M$ we can prove of Proposition 4.5.

- First, as $\mu_{Z}>0$, for any $\delta$, there exists $V_{0}>v^{*}$ independent of $\delta$, and $M(v, \delta)<0$ for $v>V_{0}$.

- As the bifurcation is subcritical, $\frac{\partial^{2}}{\partial v^{2}} M\left(v^{*}, \delta_{\mathcal{H}}\right)>0$ and therefore the point $v=v^{*}$ is a minimum of $M\left(v, \delta_{\mathcal{H}}\right)$ and $M\left(v, \delta_{\mathcal{H}}\right)>0$ for all $v^{*}<v<V_{1}$, and $\left(0, v^{*}\right)$ is unstable at the Hopf bifurcation.

- As in the invisible-invisible case $\frac{\partial^{2}}{\partial v \partial \delta} M\left(v^{*}, \delta_{\mathcal{H}}\right)>0$ one has that 
- If $\delta<\delta_{\mathcal{H}}, \frac{\partial M}{\partial v}\left(v^{*}, \delta\right)<0$, and consequently $M\left(v^{*}, \delta\right)<0$ for $v>v^{*}$ close enough to $v^{*}$, which implies that the critical point of the system is stable.

- If $\delta>\delta_{\mathcal{H}}, \frac{\partial M}{\partial v}\left(v^{*}, \delta\right)>0$, and consequently $M\left(v^{*}, \delta\right)>0$ for $v>v^{*}$ close enough to $v^{*}$, which implies that the critical point of the system is unstable.

All this information together ensures that the function $M(v, \delta)$ satisfies:

- For $\delta>\delta_{\mathcal{H}}, M(v, \delta)>0$, for any $v^{*}<v<V_{1}$, therefore $Z_{\varepsilon}^{\alpha}$ has no periodic orbits near the critical point $P(\alpha, \varepsilon)$. Nevertheless, as $M(v, \delta)<0$ for $v>V_{0}$, the function $M(\cdot, \delta)$ has a zero $v^{s}$, corresponding to an attracting periodic orbit $\Gamma_{\varepsilon}^{\alpha, s}$.

- For $\delta<\delta_{\mathcal{H}}, M(v, \delta)<0$, for $v>v^{*}$ near $v^{*}$, but is positive near $V_{1}$, therefore it has a unique zero $v^{u}=v^{u}(\delta)$ near $v^{*}$ satisfying $\frac{\partial}{\partial v} M\left(v^{u}(\delta), \delta\right)>0$, therefore, by the implicit function theorem, $Z_{\varepsilon}^{\alpha}$ has an repelling periodic orbit $\Delta_{\varepsilon}^{\alpha, u}$. In addition, as $M(v, \delta)$ is negative near $V_{0}$, it has a zero $v^{s}$, corresponding to an attracting periodic orbit $\Gamma_{\varepsilon}^{\alpha, s}$. Therefore, $M(v, \delta)$ has a maximum $v_{M}(\delta) \in\left(v^{u}, v^{s}\right)$.

- In addition, as $M$ has also a minimum between $v^{*}$ and $v^{u}$, we can assure that its second derivative vanishes, at least, in one point. If we assume that $\frac{\partial^{3}}{\partial v^{3}} M\left(v_{\mathcal{S}}, \delta_{\mathcal{S}}\right) \neq 0$, we can ensure that there are no more zeros of $M$ besides $v^{*}, v^{u}, v^{s}$.

This guarantees the existence of a pair $\left(v_{\mathcal{S}}, \delta_{\mathcal{S}}\right)$ with $v^{*}<v_{\mathcal{S}}=v_{M}\left(\delta_{\mathcal{S}}\right)$ and $\delta_{\mathcal{S}}<\delta_{\mathcal{H}}$ such that $M\left(v_{\mathcal{S}}, \delta_{\mathcal{S}}\right)=0, \frac{\partial}{\partial v} M\left(v_{\mathcal{S}}, \delta_{\mathcal{S}}\right)=0$, giving raise to a saddle-node bifurcation of periodic orbits.

The reasoning for the visible-invisible case is analogous, using that, for $\delta>\delta_{\mathcal{C}}$ the Melnikov function is also negative for $v$ near $\bar{v}$.

Even if the saddle-node bifurcation can not be computed analytically, it is worth to mention that we can use the Melnikov function to find it numerically solving the system of equations:

$$
M(v, \delta)=0, \quad \frac{\partial M}{\partial v}(v, \delta)=0 .
$$

Observe that $M$ is linear in $\delta$ and therefore one can easily reduce this system to the problem of looking for zeros of a function of one variable. This can be a useful computational tool to find the saddle-node bifurcations in the regularization for a concrete system.

\section{Acknowledgments}

C. Bonet-Reves and Tere M. Seara have been partially supported by the Spanish MINECOFEDER Grants MTM2015-65715-P and MDM-2014-0445, and the Catalan Grant 2014SGR504. Tere M-Seara is also supported by the Russian Scientific Foundation grant 14-41-00044 and the European Marie Curie Action FP7-PEOPLE-2012-IRSES: BREUDS.

J. Larrosa has been supported by FAPESP grants 2011/22529-8 and 2014/13970-0 and the European Marie Curie Action FP7-PEOPLE-2012-IRSES: BREUDS.

\section{Appendix}

\subsection{Proof of Proposition 4.2}

To prove Proposition 4.2 one need the following lemmas.

Lemma 7.1. Let $\phi_{+}^{\alpha, \varepsilon}$ the map which goes to the section $v=1$ to the section $v=-1$ and $\phi_{-}^{\alpha, \varepsilon}$ the map which goes to the section $v=-1$ to the section $v=+1$, then

(a) There exists $x^{+}(\alpha, \varepsilon)$ such that for $x>x^{+}(\alpha, \varepsilon)$ the map $\phi_{+}^{\alpha, \varepsilon}$ is well defined and $\phi_{+}^{\alpha, \varepsilon}(x)=$ $x+g^{+}(x ; \alpha) \varepsilon+\mathcal{O}\left(\varepsilon^{2}\right)$ where $g^{+}(x ; \alpha)$ is given in (7.6). 
(b) There exists $x^{-}(\alpha, \varepsilon)$ such that for $x<x^{-}(\alpha, \varepsilon)$ the map $\phi_{-}^{\alpha, \varepsilon}$ is well defined and $\phi_{-}^{\alpha, \varepsilon}(x)=$ $x+g^{-}(x ; \alpha) \varepsilon+\mathcal{O}\left(\varepsilon^{2}\right)$ where $g^{-}(x ; \alpha)=-g^{+}(x ; \alpha)$.

Proof. We prove item $(a)$, the reasoning to prove item $(b)$ is analogous.

Since the origin is an invisible fold point of $X$, fixing $v \in(-1,1)$, it follows that there exist $\alpha_{0}$ and $\varepsilon_{0}$ and a map $x(\alpha, \varepsilon v)$ such that $X^{\alpha 2}(x(\alpha, \varepsilon v), \varepsilon v)=0$ and $X^{\alpha 1} \cdot X_{x}^{\alpha 2}(x(\alpha, \varepsilon v), \varepsilon v)<0$ for every $|\alpha|<\alpha_{0}$ and $\varepsilon<\varepsilon_{0}$. For each $\alpha$ and $\varepsilon$ fixed, set

$$
x_{X}^{+}(\alpha, \varepsilon)=\max _{v \in[-1,1]} x(\alpha, \varepsilon v) .
$$

Observe that for $x>x_{X}^{+}(\alpha, \varepsilon)$ we have $X^{\alpha 2}(x, \varepsilon v)<0$ for all $v \in[-1,1]$. Using the same arguments, one can define $x_{Y}^{+}(\alpha, \varepsilon)$ in an analogous way.

Define

$$
x^{+}(\alpha, \varepsilon)=\max \left\{x_{X}^{+}(\alpha, \varepsilon), x_{Y}^{+}(\alpha, \varepsilon)\right\} .
$$

Therefore, for $x>x^{+}(\alpha, \varepsilon)$ we have that $X^{\alpha 2}(x, \varepsilon v)$ and $Y^{\alpha 2}(x, \varepsilon v)$ are smaller than zero, simultaneously. This implies that the $Z_{\varepsilon}^{\alpha}$ trajectory of any initial condition $x>x^{+}(\alpha, \varepsilon)$ crosses the regularization zone, since in this region

$$
\dot{v}=\frac{1}{\varepsilon} F^{2}(x, v ; \alpha, \varepsilon)=(1+\varphi(v)) X^{\alpha 2}(x, \varepsilon v)+(1-\varphi(v)) Y^{\alpha 2}(x, \varepsilon v)<0, \quad \forall v \in[-1,1] .
$$

Now we are able to compute the map $\phi_{+}^{\alpha \varepsilon}$. Consider the equation of the orbits of system $(3.7)$

$$
\frac{d x}{d v}=\varepsilon \frac{F^{1}(x, v ; \alpha, \varepsilon)}{F^{2}(x, v ; \alpha, \varepsilon)}
$$

Let $x(v ; \varepsilon)$ be the solution of $(7.3)$ satisfying $x(1, \varepsilon)=x_{0}>x^{+}(\alpha, \varepsilon)$. Taylor expanding this solution we obtain:

$$
x(v ; \varepsilon)=x_{0}+\varepsilon \int_{1}^{v} \frac{F^{1}\left(x_{0}, s ; \alpha, 0\right)}{F^{2}\left(x_{0}, s ; \alpha, 0\right)} d s+\mathcal{O}\left(\varepsilon^{2}\right) .
$$

Then the intersection between the $Z_{\varepsilon}^{\alpha}$ trajectory departing from $(x, 1)$ with $x>x^{+}(\alpha, \varepsilon)$ with the section $v=-1$ is given by

$$
\phi_{+}^{\alpha \varepsilon}(x)=x+g^{+}(x ; \alpha) \varepsilon+\mathcal{O}\left(\varepsilon^{2}\right)
$$

where

$$
g^{+}(x ; \alpha)=\int_{1}^{-1} \frac{F^{1}(x, s ; \alpha, 0)}{F^{2}(x, s ; \alpha, 0)} d s .
$$

Observe that the function $g^{+}$is regular respect to $\alpha: g^{+}(x ; \alpha)=g^{+}(x ; 0)+\mathcal{O}(\alpha)$.

Lemma 7.2. For $\alpha, \varepsilon>0$ sufficiently small there exists a domain $\Theta_{\varepsilon}^{\alpha}$, given in (7.11), where the generalized first return map $\phi_{\varepsilon}^{\alpha}$ is well defined and satisfies

$$
\phi_{\varepsilon}^{\alpha}=\phi^{\alpha}+\mathcal{O}(\varepsilon)=\phi_{Z}+\mathcal{O}(\alpha, \varepsilon)
$$

Proof. To fix ideas, lets assume that $\mu_{Z}>0$. The first step is to construct a first return map $\phi_{\varepsilon}^{\alpha}$ (see Figure 37) defined in a section $\Theta_{\varepsilon}^{\alpha} \subset\left\{(x, 1): x<T_{X}^{\alpha, \varepsilon}\right\}$ as follows.

Using Proposition 2.12 applied to the vector field $X^{\alpha}(x, \varepsilon v)$, we define the Poincaré map $\phi_{X}^{\alpha, \varepsilon}$ in the section $v=1$ for $x<T_{X}^{\alpha, \varepsilon}$ by

$$
\phi_{X}^{\alpha, \varepsilon}(x)=2 T_{X}^{\alpha, \varepsilon}-x+\beta_{X}^{\alpha, \varepsilon}\left(x-T_{X}^{\alpha, \varepsilon}\right)^{2}+\mathcal{O}\left(x-T_{X}^{\alpha, \varepsilon}\right)^{3} .
$$

Analogously, we have the map $\phi_{Y}^{\alpha, \varepsilon}$ defined at the section $v=-1$ for $x>T_{Y}^{\alpha, \varepsilon}$, given by

$$
\phi_{Y}^{\alpha, \varepsilon}(x)=2 T_{Y}^{\alpha, \varepsilon}-x+\beta_{Y}^{\alpha, \varepsilon}\left(x-T_{Y}^{\alpha, \varepsilon}\right)^{2}+\mathcal{O}\left(x-T_{Y}^{\alpha, \varepsilon}\right)^{3} .
$$




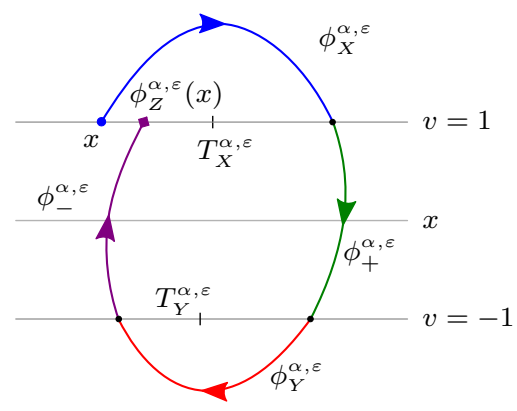

Figure 37: The generalized first return map $\phi_{X}^{\alpha \varepsilon}$

Where $\beta_{X, Y}^{\alpha, \varepsilon}$ and $T_{X, Y}^{\alpha, \varepsilon}$ are given by formulas (2.11) and (4.1) respectively. Moreover,

$$
T_{X, Y}^{\alpha, \varepsilon}=T_{X, Y}^{\alpha}+\mathcal{O}(\varepsilon) \text { and } \beta_{X, Y}^{\alpha, \varepsilon}=\beta_{X, Y}^{\alpha}+\mathcal{O}(\varepsilon)
$$

Therefore, we conclude that $\phi_{X, Y}^{\alpha, \varepsilon}=\phi_{X, Y}^{\alpha}+\mathcal{O}(\varepsilon)$, where $\phi_{X, Y}^{\alpha}$ are the Poincaré maps defined for the vector fields $X^{\alpha}, Y^{\alpha}$.

In this way, we obtain a generalized first return map $\phi_{\varepsilon}^{\alpha}$ defined in $\Theta_{\alpha, \varepsilon}$

$$
\phi_{\varepsilon}^{\alpha}=\phi_{-}^{\alpha, \varepsilon} \circ \phi_{Y}^{\alpha, \varepsilon} \circ \phi_{+}^{\alpha, \varepsilon} \circ \phi_{X}^{\alpha, \varepsilon}=\phi^{\alpha}+\mathcal{O}(\varepsilon)=\phi_{Z}+\mathcal{O}(\alpha, \varepsilon)
$$

where $\phi^{\alpha}$ is given in (2.26) and

$$
\Theta_{\alpha, \varepsilon}=\left\{(x, 1): x<\min \left\{\left(\phi_{X}^{\alpha, \varepsilon}\right)^{-1}\left(x^{+}(\alpha, \varepsilon)\right),\left(\phi_{Y}^{\alpha, \varepsilon} \circ \phi_{+}^{\alpha, \varepsilon} \circ \phi_{X}^{\alpha, \varepsilon}\right)^{-1}\left(x^{-}(\alpha, \varepsilon)\right)\right\}\right\} .
$$

Proof of Proposition 4.2. (a) By Corolary 3.8, for $\alpha>\alpha_{\mathcal{H}}$, and $\varepsilon$ small enough, the system $Z_{\varepsilon}^{\alpha}$ has an unstable focus. As $\mu_{Z}>0$ the map $\phi_{Z}$ is attracting, therefore using (7.7), $\phi_{\varepsilon}^{\alpha}$ is attracting for $a \leq x \leq b<0$. Therefore, the Poincaré-Bendixson Theorem guarantees the existence of a stable orbit $\Gamma_{\varepsilon}^{\alpha}$ for $(\alpha, \varepsilon)$ on the right of the curve $\mathcal{H}$.

(b1) By Proposition 2.18, if $\alpha>0$, the map $\phi^{\alpha}$ has a hyperbolic fixed point $F(\alpha)$ given in (2.28). Then the result is a consequence of the Implicit Function Theorem.

(b2) When $\alpha<0$ the map $\phi^{\alpha}$ has no fixed points and, by continuity, there are no fixed points for $\phi_{\varepsilon}^{\alpha}$ when $\varepsilon>0$ is sufficiently small.

(c1) Using the properties of the Melnikov function given in Proposition 5.1 we have:

- $M\left(v^{*}, \delta\right)=0$.

- As $\mu_{Z}>0$, for any $\delta$, there exists $V_{0}>v^{*}$ independent of $\delta$, and $M(v, \delta)<0$ for $v>V_{0}$.

- As in the invisible-invisible case $\frac{\partial^{2}}{\partial v \partial \delta} M\left(v^{*}, \delta_{\mathcal{H}}\right)>0$ one has that

- If $\delta<\delta_{\mathcal{H}}, \frac{\partial M}{\partial v}\left(v^{*}, \delta\right)<0$, and consequently $M\left(v^{*}, \delta\right)<0$ for $v>v^{*}$ close enough to $v^{*}$, which implies that the critical point of the system is stable.

- If $\delta>\delta_{\mathcal{H}}, \frac{\partial M}{\partial v}\left(v^{*}, \delta\right)>0$, and consequently $M\left(v^{*}, \delta\right)>0$ for $v>v^{*}$ close enough to $v^{*}$, which implies that the critical point of the system is unstable.

(c2) For $\delta>\delta_{\mathcal{H}}$ Bolzano Theorem assures that the Melnikov function has a zero $v^{*}<v^{s}=v^{s}(\delta)<$ $V_{0}$ satisfying $\frac{\partial}{\partial v} M\left(v^{s}(\delta)\right) \leq 0$ corresponding to the attracting periodic orbit $\Gamma_{\varepsilon}^{\alpha, s}$. Moreover, $\Gamma_{\varepsilon}^{\alpha, s}$ is locally unique if $\frac{\partial}{\partial v} M\left(v^{s}, \delta_{\mathcal{H}}\right)<0$.

(c3) If we assume that $M(v, \delta)$ is strictly concave, no more zeros of $M(v, \delta)$ exist for $\delta>\delta_{\mathcal{H}}$ and the periodic orbit $\Gamma_{\varepsilon}^{\alpha, s}$ is unique. Analogously, for $\delta<\delta_{\mathcal{H}}$ the function $M(v, \delta)<0$ for $v^{*}<v$. 


\subsection{Proof of Proposition 4.6}

During this section we restrict ourselves to any compact set containing the folds and the results will be valid for $\varepsilon$ small enough depending of this fixed compact.

First, as numerical simulations indicate that there is a maximal Canard trajectory when $\alpha=$ $\mathcal{O}(\varepsilon)$, we set $\alpha=\delta \varepsilon$. Therefore, system (3.5) becomes

$$
\begin{aligned}
\dot{x} & =F^{1}(x, v ; \delta \varepsilon, \varepsilon) \\
\varepsilon \dot{v} & =F^{2}(x, v ; \delta \varepsilon, \varepsilon)
\end{aligned}
$$

As $\alpha=\delta \varepsilon$, the critical manifolds of this system are the same as the ones for the regularization of the vector field $Z$. Then, as we saw in subsubsection 3.2.2 there are two critical manifolds $\Lambda_{0}^{s, u}$ given by:

$$
\Lambda_{0}^{s}=\left\{(x, v), v=m_{0}(x), x<0\right\}, \Lambda_{0}^{u}=\left\{(x, v), v=m_{0}(x), x>0\right\}
$$

where

$$
m_{0}(x)=-\varphi^{-1}\left(\frac{X^{2}+Y^{2}}{X^{2}-Y^{2}}(x, 0)\right),
$$

which are normally hyperbolic (attracting and repelling respectively) and we restrict them to $|x|>\kappa$ for a small but fixed $\kappa>0$. Applying Fenichel theorem, we know the existence of two normally hyperbolic invariant manifolds

$$
\Lambda_{\varepsilon}^{s}=\left\{(x, v), v=m^{s}(x ; \varepsilon), x<-\kappa\right\}, \Lambda_{\varepsilon}^{u}=\left\{(x, v), v=m^{u}(x ; \varepsilon), x>\kappa\right\} .
$$

The idea is now, to "extend" these manifolds until they reach $x=0$ and to see if they can coincide for some value of $\delta$ giving rise to the so-called maximal Canards.

We first look for the asymptotic expansion of the functions $m^{s, u}(x ; \varepsilon)$ defining these manifolds:

$$
m^{s, u}(x ; \varepsilon)=m_{0}(x)+m_{1}(x) \varepsilon+m_{2}(x) \varepsilon^{2}+\mathcal{O}\left(\varepsilon^{3}\right) .
$$

Using that $m^{s, u}(x ; \varepsilon)$ satisfy the equation of the orbits:

$$
\varepsilon \frac{d v}{d x}=\frac{F^{2}(x, v ; \delta \varepsilon, \varepsilon)}{F^{1}(x, v ; \delta \varepsilon, \varepsilon)}
$$

we can obtain analytical expressions for $m_{i}(x)$ for $i=0,1, \cdots$, with $m_{0}(x)$ given in Equation (7.13), and it is easy to check that they behave as:

$$
m_{0}(x)=\mathcal{O}(1), m_{1}(x)=\mathcal{O}\left(\frac{1}{x}\right), m_{2}(x)=\mathcal{O}\left(\frac{1}{x^{3}}\right)
$$

Therefore while $|x|<\sqrt{\varepsilon}$ the series (7.14) is asymptotic. Next proposition gives the behavior of the stable Fenichel manifold $m^{s}(x ; \varepsilon)$ shown in these expansions. Analogously, one can prove the same result for the unstable Fenichel manifold $m^{u}(x ; \varepsilon)$ reversing time.

Proposition 7.3. Take any $0<\lambda<\frac{1}{2}$. Fix $x_{0}>\kappa$. Then, there exists $M>0$ big enough, $\sigma=\sigma(M)>0$ small enough, and $\varepsilon_{0}=\varepsilon_{0}(M, \sigma)>0$ such that, for $0<\varepsilon<\varepsilon_{0}$, any solution of system (3.5) which enters the set

$$
B=\left\{(x, v):-x_{0} \leq x \leq-\varepsilon^{\lambda},\left|v-m_{0}(x)\right| \leq \frac{M \varepsilon}{|x|}\right\}
$$

leaves it through the boundary $x=-\varepsilon^{\lambda}$.

Proof. The proof is based in the fact that the vector field $\tilde{Z}_{\varepsilon}^{\alpha}(3.5)$ points inwards through the boundaries

$$
B^{ \pm}=\left\{(x, v): \quad-x_{0} \leq x \leq-\varepsilon^{\lambda}, v=m_{0}(x) \mp \frac{M \varepsilon}{x}\right\} .
$$


This is straightforward and can be seen in full details in [RS14]. For instance, to see that the vector field $\tilde{Z}_{\varepsilon}^{\alpha}$ points inwards $B$ through $B^{+}$, we must see that

$$
\left\langle\left(-m_{0}^{\prime}(x)-\frac{M \varepsilon}{x^{2}}, 1\right), \tilde{Z}_{\varepsilon}^{\alpha}\right\rangle<0, \quad-x_{0} \leq x \leq-\varepsilon^{\lambda}
$$

and for this purpose it will be enough to see that, in fact, the negative sign of the coefficient of the $\varepsilon$ order of this expression determines the sign of it.

Next step is to enlarge the domain of existence of these stable and unstable Fenichel manifolds. We take a value $x^{*}=-\varepsilon^{\lambda}$ for the stable case (or $x^{*}=\varepsilon^{\lambda}$ for the unstable case), with $0<\lambda<1 / 2$. We know that

$$
\left|m^{s, u}\left(x^{*} ; \varepsilon\right)-m_{0}\left(x^{*}\right)\right| \leq M \varepsilon^{1-\lambda} .
$$

It is clear that (7.14) looses its asymptotic character when $|x|=\varepsilon^{\frac{1}{2}}$. This suggests the change $x=\varepsilon^{\frac{1}{2}} r$. Now the Taylor expansion of the Fenichel manifold in this new variable justifies the change $v=\bar{v}+\varepsilon^{\frac{1}{2}} s$, where $\bar{v}=m_{0}(0)$ (see Equation (3.26).

Finally, setting $\gamma=\varepsilon^{\frac{1}{2}}$, we study the continuation of the Fenichel manifolds for small values of $x$, performing the following change to system (3.5).

$$
\left\{\begin{array}{l}
x=\gamma r \\
v=\bar{v}+\gamma s
\end{array}\right.
$$

With this change and re-scaling time $t=\gamma \tau$, system (7.12) becomes

$$
\left\{\begin{array}{l}
\dot{r}=M_{0}+\mathcal{O}(\gamma), \\
\dot{s}=M_{1}+M_{2} \delta+M_{3} r^{2}+M_{4} r s+\mathcal{O}(\gamma),
\end{array}\right.
$$

where $\mathcal{O}(\gamma)$ are terms bounded by $K \gamma$, where $K>0$ is independent of $\gamma$, for $r^{*}<r \leq 0$, where $r^{*}=\frac{x^{*}}{\sqrt{\varepsilon}}=-\gamma^{2 \lambda-1}$ and the constants are given by

$$
\begin{aligned}
& M_{0}=\left(X^{1}+Y^{1}+\varphi(\bar{v})\left(X^{1}-Y^{1}\right)\right)(\mathbf{0}), \\
& M_{1}=\bar{v}\left(X_{y}^{2}+Y_{y}^{2}+\varphi(\bar{v})\left(X_{y}^{2}-Y_{y}^{2}\right)\right)(\mathbf{0}), \\
& M_{2}=\left(\tilde{X}^{2}+\tilde{Y}^{2}+\varphi(\bar{v})\left(\tilde{X}^{2}-\tilde{Y}^{2}\right)\right)(\mathbf{0}), \\
& M_{3}=\frac{1}{2}\left(X_{x x}^{2}+Y_{x x}^{2}+\varphi(\bar{v})\left(X_{x x}^{2}-Y_{x x}^{2}\right)\right)(\mathbf{0}), \\
& M_{4}=\varphi^{\prime}(\bar{v})\left(X_{x}^{2}-Y_{x}^{2}\right)(\mathbf{0}) .
\end{aligned}
$$

Putting $\gamma=0$ in system (7.19) we obtain

$$
\left\{\begin{array}{l}
\dot{r}=M_{0}, \\
\dot{s}=M_{1}+M_{2} \delta+M_{3} r^{2}+M_{4} r s,
\end{array}\right.
$$

which gives us the so called inner equation

$$
\frac{d s}{d r}=N_{1}+N_{2} \delta+N_{3} r^{2}+N_{4} r s
$$

where $N_{i}=\frac{M_{i}}{M_{0}}$ for $i=1,2,3,4$.

Observe that since $M_{0}=\frac{-(\operatorname{det} Z)_{x}(\mathbf{0})}{\left(X_{x}^{2}-Y_{x}^{2}\right)(\mathbf{0})}>0$, then $N_{4}=\varphi^{\prime}(\bar{v}) \frac{\left(X_{x}^{2}-Y_{x}^{2}\right)^{2}(\mathbf{0})}{-(\operatorname{det} Z)_{x}(\mathbf{0})}>0$. 
From now, one must find a solution of system (7.19) which coincides with the Fenichel manifold at the point $x^{*}=\gamma r^{*}$. Let us recall that $\varepsilon=\gamma^{2}$ and that $r^{*}=-\gamma^{2 \lambda-1}$ for the stable case and $r^{*}=\gamma^{2 \lambda-1}$ for the unstable case.

In the new variables, the Fenichel manifolds satisfy

$$
s^{s, u}\left(r^{*} ; \gamma\right)=\frac{m^{s, u}\left(x^{*}\right)-\bar{v}}{\gamma}=m_{0}^{\prime}(0) r^{*}+O\left(\gamma^{1-2 \lambda}, \gamma^{4 \lambda-1}\right),
$$

and this suggests to look for solutions $s_{i}^{\mp}(r)$ of the inner equation (7.22) satisfying:

$$
\left|s_{0}^{\mp}(r)-m_{0}^{\prime}(0) r\right| \text { bounded as } r \rightarrow \mp \infty .
$$

As $N_{4}>0$, these particular solutions are

$$
\begin{aligned}
& s_{0}^{-}(r)=e^{\frac{1}{2} N_{4} r^{2}} \int_{-\infty}^{r} e^{-\frac{1}{2} N_{4} t^{2}}\left(N_{1}+N_{2} \delta+N_{3} t^{2}\right) d t, \\
& s_{0}^{+}(r)=-e^{\frac{1}{2} N_{4} r^{2}} \int_{r}^{\infty} e^{-\frac{1}{2} N_{4} t^{2}}\left(N_{1}+N_{2} \delta+N_{3} t^{2}\right) d t .
\end{aligned}
$$

Integrating by parts and using the expression for $M_{3}$ and $M_{4}$ given in (7.20) and the expression for $m_{0}(x)$ given in (7.13), we get:

$$
\begin{aligned}
& s_{0}^{-}(r)=m_{0}^{\prime}(0) r+\left(N_{1}+N_{2} \delta+\frac{N_{3}}{N_{4}}\right) \int_{-\infty}^{r} e^{\frac{1}{2} N_{4}\left(r^{2}-t^{2}\right)} d t, \\
& s_{0}^{+}(r)=m_{0}^{\prime}(0) r-\left(N_{1}+N_{2} \delta+\frac{N_{3}}{N_{4}}\right) \int_{r}^{\infty} e^{\frac{1}{2} N_{4}\left(r^{2}-t^{2}\right)} d t .
\end{aligned}
$$

Using the L'Hôpital Rule one can easily see that $s_{0}^{ \pm}(r)-m_{0}^{\prime}(0) r$ tend to zero when $r \rightarrow \pm \infty$. More concretely:

$$
s_{0}^{\mp}(r)-m_{0}^{\prime}(0) r=\mathcal{O}\left(\frac{1}{r}\right), r \rightarrow \pm \infty .
$$

Analogously to what we did in the study of the stable Fenichel manifold in the region $-x_{0}<$ $x<-\varepsilon^{\lambda}$ (and the unstable one for $\varepsilon^{\lambda}<x<x_{0}$ ), we seek solutions of (7.19) in the form

$$
s^{u, s}(r, \gamma)=s_{0}^{ \pm}(r)+\gamma s_{1}^{ \pm}(r)+\cdots
$$

Substituting this expression in (7.19), we obtain that the successive $s_{i}^{ \pm}$satisfy linear equations with the same homogeneous part as the one satisfied by $s_{0}^{ \pm}(r)$, only differing on the non homogeneous term which depends recursively of $s_{i-1}^{ \pm}$. Obviously, if we want to follow the Fenichel manifolds, we must select for $s_{i}^{ \pm}$the solution with no exponential term. So, as we did for $s_{0}^{ \pm}$, the L'Hôpital Rule shows that

$$
s_{1}^{ \pm}(r)=\mathcal{O}\left(r^{2}\right)
$$

This suggests, like in Proposition 7.3, the definition of a new block $\mathcal{B}$ for the equation (7.19). Also, to see that effectively the continuation of the Fenichel manifold is well approximated by $s_{0}^{\mp}$ for $r^{*} \leq r \leq 0$ we have the following proposition for the behavior of the stable part. Analogously, reversing time, one can prove the same result for the unstable one.

Proposition 7.4. Take any $\frac{1}{4}<\lambda<\frac{1}{2}$. Then, there exists $r_{0}>0, K>0$ and $\gamma_{0}=\gamma_{0}\left(r_{0}, K\right)$, such that for $\gamma \leq \gamma_{0}$, any solution of system (7.19) which enters the set

$$
\mathcal{B}=\left\{(r, s): r^{*} \leq r \leq 0,\left|s-s_{0}^{-}(r)\right| \leq K \gamma M(r)\right\}
$$

where $r^{*}=-\gamma^{2 \lambda-1}$ and the function $M(r)$ is defined by

$$
M(r)=\left\{\begin{array}{ll}
r^{2}, & -\infty \leq r \leq-r_{0}<0 \\
r_{0}^{2}, & -r_{0} \leq r<0
\end{array} .\right.
$$

leaves it through the boundary $r=0$. 
Proof. We proceed as in Proposition 7.3 to see that the vector field (7.19) points inwards through the boundaries

$$
\mathcal{B}^{ \pm}=\left\{(r, s): \quad r^{*} \leq r \leq 0, s=s_{0}^{-}(r) \pm K \gamma M(r)\right\} .
$$

This is straightforward and details can be found in [RS14]. Only to remark that $r_{0}$ can be large, but now the vector field (7.19) is regular in $\gamma$, so the behavior of the Fenichel manifolds from $r_{0}$ to the origin is guaranteed to be $\mathcal{O}(\gamma)$.

To see that the Fenichel manifold enters the block $\mathcal{B}$ we use estimates (7.23) and (7.26) and taking $\frac{1}{4}<\lambda<\frac{1}{3}$ we obtain the result.

Using $s_{0}^{\mp}$, we can continue the Fenichel manifolds until we reach $r=0$ :

$$
v^{s, u}(x, \varepsilon)=\bar{v}+\gamma s^{u, s}(r ; \gamma)=\bar{v}+\gamma s_{0}^{ \pm}(r)+\mathcal{O}\left(\gamma^{2}\right), r=\frac{x}{\gamma}, \varepsilon=\gamma^{2},
$$

and the intersection with $x=0$ is given by:

$$
v^{s, u}(0, \varepsilon)=\bar{v}+\gamma s_{0}^{ \pm}(0)+\mathcal{O}\left(\gamma^{2}\right) .
$$

And we obtain:

$$
\begin{aligned}
v^{s}(0, \varepsilon)-v^{u}(0, \varepsilon) & =\gamma\left(s_{0}^{-}(0)-s_{0}^{+}(0)\right)+\mathcal{O}\left(\gamma^{2}\right)=\gamma\left(N_{1}+N_{2} \delta+\frac{N_{3}}{N_{4}}\right) \int_{-\infty}^{\infty} e^{-\frac{1}{2} N_{4} t^{2}}+\mathcal{O}\left(\gamma^{2}\right) \\
& =\gamma\left(N_{1}+N_{2} \delta+\frac{N_{3}}{N_{4}}\right) \sqrt{\frac{2 \pi}{N_{4}}}+\mathcal{O}\left(\gamma^{2}\right)
\end{aligned}
$$

Calling $\xi(\delta, \gamma)=\frac{1}{\gamma}\left(v^{s}-v^{u}\right)$ one has that $\xi\left(\delta_{\mathcal{C}}, 0\right)=0$, where:

$$
\delta_{\mathcal{C}}=-\frac{N_{3}+N_{1} N_{4}}{N_{2} N_{4}}=-\frac{M_{0} M_{3}+M_{1} M_{4}}{M_{2} M_{4}}
$$

where $M_{i}, i=0,1,2,3,4$ are given in $(7.20)$. Now, using that $\frac{\partial \xi}{\partial \delta}\left(\delta_{\mathcal{C}}, 0\right)=N_{2} \sqrt{\frac{2 \pi}{N_{4}}} \neq 0$ (see Remark 7.5 one can apply the Implicit Function Theorem, obtaining a curve

$$
\delta_{\mathcal{C}}(\gamma)=\delta_{\mathcal{C}}+\mathcal{O}(\gamma),
$$

such that over this curve the trajectories $s^{s}(r ; \gamma)$ and $s^{u}(r ; \gamma)$ coincide. Moreover, for $\delta=\delta_{\mathcal{C}}$, one has, for $r=\mathcal{O}(1)$ (and $x=\mathcal{O}(\sqrt{\varepsilon})$ ):

$$
s_{0}^{\mp}(r)=m_{0}^{\prime}(0) r, \quad v^{s, u}(x)=m_{0}(0)+m_{0}^{\prime}(0) x+\mathcal{O}(\varepsilon) .
$$

Coming back to the original variables $(x, v)$ and recalling that $\alpha=\delta \varepsilon$, we have a curve

$$
\alpha_{\mathcal{C}}(\varepsilon)=\delta_{\mathcal{C}} \varepsilon+\mathcal{O}\left(\varepsilon^{\frac{3}{2}}\right)
$$

where $\Lambda_{\varepsilon}^{\alpha, u}$ and $\Lambda_{\varepsilon}^{\alpha, s}$ coincide.

In conclusion, there exists a curve $\mathcal{C}$ given by

$$
\mathcal{C}=\left\{(\alpha, \varepsilon): \alpha=\alpha_{\mathcal{C}}(\varepsilon)=-\frac{M_{0} M_{3}+M_{1} M_{4}}{M_{2} M_{4}} \varepsilon+\mathcal{O}\left(\varepsilon^{\frac{3}{2}}\right)\right\},
$$

where $\Lambda_{\varepsilon}^{\alpha, u}=\Lambda_{\varepsilon}^{\alpha, s}$ giving rise to a Canard solution. Moreover, as $s_{0}(0)=0$, at this value one has that:

$$
v^{s, u}(0, \varepsilon)=\bar{v}+\mathcal{O}(\varepsilon) .
$$

Finally, observe that, for any $\delta$ we obtain:

$$
v^{s}-v^{u}=\gamma C\left(\delta-\delta_{\mathcal{C}}\right)+\mathcal{O}\left(\gamma^{2}\right) .
$$

where $C=\sqrt{\frac{2 \pi}{N_{4}}} N_{2}$. 
Remark 7.5. Observe that the denominator of $\delta_{\mathcal{C}}$ given by $M_{2} M_{4}=2 \varphi^{\prime}(\bar{v})\left(\tilde{Y}^{2} X_{x}^{2}-\tilde{X}^{2} Y_{x}^{2}\right)(\mathbf{0}) \neq 0$ due to the transversality condition (see (2.19)) imposed to the unfolding $Z^{\alpha}$.

Remark 7.6. In the case $(\operatorname{det} Z)_{x}(\mathbf{0})>0$, as $N_{4}<0$, all the solutions of the inner equation have the correct asymptotic behavior as $r \rightarrow \pm \infty$, nevertheless when $\delta=\delta_{\mathcal{C}}$ the solutions $s_{0}^{\mp}(r)=m_{0}^{\prime}(0) r$ can be seen as the "Canard" solution.

\subsection{Proof of Theorem 4.10}

In this section we will prove that, for the case of a linear regularization $\varphi$ of the visible-invisible fold-fold singularity with $(\operatorname{det} Z)_{x}<0$ one can transform the system to a general slow-fast system studied in KS01a; KS01b Kue10 and apply their results for the existence of a maximal canard. We will recover all the values of $\delta_{\mathcal{H}}, \delta_{\mathcal{C}}$ computed in this paper, as well as the first Lyapunov coefficient at the Hopf bifurcation, and their relations.

In $\mathrm{KS} 01 \mathrm{a}$; KS01b], the authors proved the existence of a maximal Canard for the following general system

$$
\left\{\begin{array}{l}
\dot{x}=R^{1}(x, y, \varepsilon, \lambda)=-y h_{1}(x, y, \varepsilon, \lambda)+x^{2} h_{2}(x, y, \varepsilon, \lambda)+\varepsilon h_{3}(x, y, \varepsilon, \lambda), \\
\dot{y}=\varepsilon R^{2}(x, y, \varepsilon, \lambda)=\varepsilon\left(x h_{4}(x, y, \varepsilon, \lambda)-\lambda h_{5}(x, y, \varepsilon, \lambda)+y h_{6}(x, y, \varepsilon, \lambda)\right),
\end{array}\right.
$$

with $h_{3}(x, y, \varepsilon, \lambda)=\mathcal{O}(x, y, \varepsilon, \lambda)$ and $h_{j}(x, y, \varepsilon, \lambda)=1+\mathcal{O}(x, y, \varepsilon, \lambda)$ for $j=1,2,4,5$. Moreover, let

$$
\begin{array}{rlrl}
\gamma_{1} & =\frac{\partial}{\partial x} h_{3}(0,0,0,0), \quad \gamma_{2} & =\frac{\partial}{\partial x} h_{1}(0,0,0,0), \quad \gamma_{3}=\frac{\partial}{\partial x} h_{2}(0,0,0,0), \\
\gamma_{4} & =\frac{\partial}{\partial x} h_{4}(0,0,0,0), \quad \gamma_{5}=h_{6}(0,0,0) .
\end{array}
$$

Considering the system (7.32), they obtain:

Theorem 7.7 (Krupa-Szmolyan Theorem). For $0<\varepsilon<\varepsilon_{0},|\lambda|<\lambda_{0}, \varepsilon_{0}, \lambda_{0}$ sufficiently small and under the previous assumptions, there exists a unique critical point $P(\lambda, \varepsilon)$ of system (7.32) in a neighborhood of the origin. The equilibrium point undergoes to a Hopf bifurcation at $\lambda_{H}$ with

$$
\lambda_{H}=-\frac{\gamma_{1}+\gamma_{5}}{2} \varepsilon+\mathcal{O}\left(\varepsilon^{2}\right)
$$

The slow manifolds $C_{\varepsilon, l}$ and $C_{\varepsilon, r}$ intersect/coincide at a maximal Canard at $\lambda_{c}$ for

$$
\lambda_{c}=-\left(\frac{\gamma_{1}+\gamma_{5}}{2}+\frac{A}{8}\right) \varepsilon+\mathcal{O}\left(\varepsilon^{3 / 2}\right)
$$

where

$$
A=-\gamma_{2}+3 \gamma_{3}-2 \gamma_{4}-2 \gamma_{5} .
$$

The equilibrium $P(\lambda, \varepsilon)$ is stable for $\lambda<\lambda_{H}$ and unstable for $\lambda>\lambda_{H}$. The Hopf bifurcation is non degenerated for $A \neq 0$, supercritical for $A<0$ and subcritical for $A>0$.

Putting $\lambda=\kappa \varepsilon$ in (7.32), the next lemma is straightforward:

Lemma 7.8. In a suitable neighborhood of the origin, one has that:

- the critical manifold $C_{0}$ of system (7.32) can be written as the graph of

$$
f(x)=x^{2}+\left(\gamma_{3}-\gamma_{2}\right) x^{3}+\mathcal{O}\left(x^{4}\right)
$$


- considering the coordinate change

$$
\begin{cases}u & =x, \\ \varepsilon w & =y-f(x),\end{cases}
$$

system (7.32), becomes

$$
\left\{\begin{aligned}
\dot{u} & =\gamma_{1} u-w\left(1+\gamma_{2} u\right)+\tilde{R}^{1}(u, w, \varepsilon) \\
\varepsilon \dot{w} \quad & =u-\kappa \varepsilon+\left(\gamma_{5}+\gamma_{4}-2 \gamma_{1}\right) u^{2}+\gamma_{5} \varepsilon w+2 u w \\
& +\left(3 \gamma_{3}-\gamma_{2}\right) u^{2} w+\tilde{R}^{2}(u, w, \varepsilon)
\end{aligned}\right.
$$

with

$$
\begin{aligned}
\tilde{R}^{1}(u, w, \varepsilon) & =u^{2} K_{4}(u)+\varepsilon^{2} K_{2}(u, \varepsilon)+u^{2} w K_{5}(u)+\varepsilon w K_{3}(u, \varepsilon)+\varepsilon w^{2} K_{1}(u, \varepsilon w, \varepsilon), \\
\tilde{R}^{2}(u, w, \varepsilon) & =u^{3} K_{12}(u)+u^{3} w K_{13}(u)+f^{\prime}(u)\left(\varepsilon^{2} K_{2}(u, \varepsilon)+\varepsilon w K_{3}(u, \varepsilon)\right. \\
& \left.+\varepsilon w^{2} K_{1}(u, \varepsilon w, \varepsilon)\right)+\varepsilon^{2} K_{7}(u, \varepsilon)+\varepsilon w K_{11}(u)+\varepsilon^{2} w K_{8}(u, \varepsilon) \\
& +(\varepsilon w)^{2} K_{6}(u, \varepsilon w, \varepsilon) .
\end{aligned}
$$

Where the functions $K_{i}$ are smooth bounded functions.

Remark 7.9. Observe that system (7.38) is a slow-fast system and its critical manifold is given by

$$
\tilde{C}_{0}=\{(u, w): u=0\} \cup\left\{(u, w): w=-\frac{1}{2}+\left(-\frac{\gamma_{5}+\gamma_{4}-2 \gamma_{1}}{2}+\frac{3 \gamma_{3}-\gamma_{2}}{4}\right) u+\mathcal{O}\left(u^{2}\right)\right\} .
$$

Now suppose that $\varphi(v)=v$ for $v \in(-1,1)$ and let $Z_{\varepsilon}^{\alpha}$ be the $\varphi$-regularization of $Z^{\alpha}$ and that $\alpha=\delta \varepsilon$. The regularized system $Z_{\varepsilon}^{\alpha}$ in coordinates $(x, v)$ with $y=\varepsilon v$ has the form

$$
\begin{cases}\dot{x} & =F^{1}(x, v ; \delta \varepsilon, \varepsilon) \\ \varepsilon \dot{v} & =F^{2}(x, v ; \delta \varepsilon, \varepsilon) .\end{cases}
$$

where:

$$
F^{i}(x, v ; \alpha, \varepsilon)=\left(X^{\alpha i}+Y^{\alpha i}\right)(x, \varepsilon v)+v\left(X^{\alpha i}-Y^{\alpha i}\right)(x, \varepsilon v), i=1,2 .,|v| \leq 1
$$

Recall that for $\varepsilon=0$ system (7.39) has a critical point at $P(0,0)=\left(0, v^{*}\right)$ with

$$
v^{*}=-\frac{X^{1}+Y^{1}}{X^{1}-Y^{1}}(\mathbf{0}) .
$$

The next proposition shows that there exists a coordinate change, such that system (7.39) is equivalent to system $(7.32)$ for $\varepsilon \neq 0$.

Proposition 7.10. There exists a change of coordinates:

$$
(x, v) \rightarrow(u, w)
$$

such that transforms system (7.39) into:

$$
\left\{\begin{aligned}
\dot{u} & =\frac{A_{1}}{\sqrt{-A_{3}}} u-w\left(1+\frac{2 A_{2} \sqrt{-A_{3}}}{A_{6}} u\right)+\tilde{S}^{1}(u, w, \varepsilon), \\
\varepsilon \dot{w} & =u+\frac{A_{6}}{2 A_{3} \sqrt{-A_{3}}}\left(A_{4}+A_{6} \delta\right) \varepsilon+\frac{1}{\sqrt{-A_{3}}}\left(A_{7}+A_{8} \delta\right) \varepsilon w+2 u w \\
& -\frac{2 A_{9}}{A_{6} \sqrt{-A_{3}}} u^{2}+\frac{4 A_{10} \sqrt{-A_{3}}}{A_{6}^{2}} u^{2} w+\tilde{S}^{2}(u, w, \varepsilon),
\end{aligned}\right.
$$


where $A_{i}, i=1, \cdots, 10$ are given in expressions (7.43) to (7.52) and

$$
\begin{aligned}
\tilde{S}^{1}(u, w, \varepsilon) & =u^{2} T_{4}(u)+\varepsilon T_{2}(u, \varepsilon)+u^{2} w T_{5}(u)+\varepsilon w T_{3}(u, \varepsilon)+\varepsilon w^{2} T_{1}(u, w, \varepsilon), \\
\tilde{S}^{2}(u, w, \varepsilon) & =u^{3} T_{9}(u)+\varepsilon u T_{10}(u)+\varepsilon^{2} T_{7}(u, \varepsilon)+u^{3} w T_{11}(u)+\varepsilon u w T_{12}(u) \\
& +\varepsilon w^{2} T_{8}(w, \varepsilon)+\varepsilon w^{2} T_{6}(u, w, \varepsilon)
\end{aligned}
$$

Where the functions $T_{i}$ are smooth bounded functions.

Proof. We begin with a coordinate change which moves the critical point $P(0,0)$ to the origin, given by

$$
\begin{cases}\bar{u} & =x \\ \bar{v} & =\left(X^{1}+Y^{1}\right)(\mathbf{0})+v\left(X^{1}-Y^{1}\right)(\mathbf{0}) .\end{cases}
$$

obtaining the system

$$
\left\{\begin{aligned}
\dot{\bar{u}} & =A_{1} \bar{u}+\bar{v}\left(1+A_{2} \bar{u}\right)+\bar{u}^{2} T_{4}(\bar{u})+\varepsilon T_{2}(\bar{u}, \varepsilon)+\bar{u}^{2} \bar{v} T_{5}(\bar{u}) \\
& +\varepsilon \bar{v} T_{3}(\bar{u}, \varepsilon)+\varepsilon \bar{v}^{2} T_{1}(\bar{u}, \bar{v}, \varepsilon), \\
\varepsilon \dot{\bar{v}} & =A_{3} \bar{u}+\left(A_{4}+A_{5} \delta\right) \varepsilon+A_{6} \bar{u} \bar{v}+\left(A_{7}+A_{8} \delta\right) \varepsilon \bar{v}+A_{9} \bar{u}^{2} \\
& +A_{10} \bar{u}^{2} \bar{v}+\bar{u}^{3} T_{9}(\bar{u})+\varepsilon \bar{u} T_{10}(\bar{u})+\varepsilon^{2} T_{7}(\bar{u}, \varepsilon)+\bar{u}^{3} \bar{v} T_{11}(\bar{u}) \\
& +\varepsilon \bar{u} \bar{v} T_{12}(\bar{u})+\varepsilon \bar{v}^{2} T_{8}(\bar{u}, \varepsilon)+\varepsilon \bar{v}^{2} T_{6}(\bar{u}, \bar{v}, \varepsilon),
\end{aligned}\right.
$$

where the constants $A_{i} i=1, \ldots, 10$ are given by

$$
\begin{aligned}
A_{1} & \left.=\left(X_{x}^{1}+Y_{x}^{1}\right)(\mathbf{0})+v^{*}\left(X_{x}^{1}-Y_{x}^{1}\right)(\mathbf{0})\right), \\
A_{2} & =\frac{\left(X_{x}^{1}+Y_{x}^{1}\right)(\mathbf{0})}{\left(X^{1}-Y^{1}\right)(\mathbf{0})}, \\
A_{3} & =2(\operatorname{det} Z)_{x}(\mathbf{0}), \\
A_{4} & =2(\operatorname{det} Z)_{y}(\mathbf{0}) v^{*}, \\
A_{5} & =2\left(\tilde{Y}^{2} X^{1}-\tilde{X}^{2} Y^{1}\right)(\mathbf{0}), \\
A_{6} & =\left(X_{x}^{2}-Y_{x}^{2}\right)(\mathbf{0}), \\
A_{7} & =\frac{2(\operatorname{det} Z)_{y}(\mathbf{0})}{\left(X^{1}-Y^{1}\right)(\mathbf{0})}+\left(X_{y}^{2}-Y_{y}^{2}\right)(\mathbf{0}) v^{*}, \\
A_{8} & =\left(\tilde{X}^{2}-\tilde{Y}^{2}\right)(\mathbf{0}), \\
A_{9} & =\frac{1}{2}\left(X^{1}-Y^{1}\right)(\mathbf{0})\left(\left(X_{x x}^{2}+Y_{x x}^{2}\right)(\mathbf{0})+v^{*}\left(X_{x x}^{2}-Y_{x x}^{2}\right)(\mathbf{0})\right), \\
A_{10} & =\frac{1}{2}\left(X_{x x}^{2}-Y_{x x}^{2}\right)(\mathbf{0}) .
\end{aligned}
$$

Now, as $A_{3}<0$, we can perform the scaling

$$
\left\{\begin{array}{l}
\bar{u}=\frac{2 \sqrt{-A_{3}}}{A_{6}} u, \\
\bar{v}=\frac{2 A_{3}}{A_{6}} w, \\
\tau=\left(\sqrt{-A_{3}}\right) t,
\end{array}\right.
$$

system (7.42) becomes the desired one, given in (7.40)

Using Lemma 7.8 and Proposition 7.10 we can apply Theorem 7.7 to system (7.40), which correspond to the regularized system $Z_{\varepsilon}^{\alpha}$. Now we can finally prove Theorem 4.10 
Proof. Proof of Theorem 4.10

By Lemma 7.8 and Proposition 7.10 we have that system (7.32) and the regularized System $(7.42)$ can be identified with the following relations

$$
\begin{aligned}
\gamma_{1} & =\frac{A_{1}}{\sqrt{-A_{3}}}, \\
\gamma_{2} & =\frac{2 A_{2} \sqrt{-A_{3}}}{A_{6}}, \\
\gamma_{5} & =\frac{1}{\sqrt{-A_{3}}}\left(A_{7}+A_{8} \delta\right), \\
\gamma_{4} & =-\frac{2 A_{9}}{A_{6} \sqrt{-A_{3}}}-\gamma_{5}+2 \gamma_{1}, \\
3 \gamma_{3} & =\frac{4 A_{10} \sqrt{-A_{3}}}{A_{6}^{2}}+\gamma_{2}, \\
\kappa & =-\frac{A_{6}}{2 A_{3} \sqrt{-A_{3}}}\left(A_{4}+A_{5} \delta\right) .
\end{aligned}
$$

Recall that in our case, the parameter $\lambda$ from Theorem 7.7 is $\lambda=\kappa \varepsilon$, thus the critical point undergoes to a Hopf bifurcation at $\lambda_{H}=\kappa_{H} \varepsilon$.

$$
\lambda_{H}=\kappa_{H} \varepsilon=-\frac{\gamma_{1}+\gamma_{5}}{2} \varepsilon+\mathcal{O}\left(\varepsilon^{2}\right)
$$

Then the value of $\kappa$ in which that Hopf bifurcation occurs is

$$
\kappa_{H}=-\frac{\gamma_{1}+\gamma_{5}}{2}+\mathcal{O}(\varepsilon)
$$

Moreover, combining expressions (7.59) and (7.60), it follows that

$$
-\frac{1}{2 \sqrt{-A_{3}}}\left(\frac{A_{4} A_{6}}{A_{3}}+\frac{A_{5} A_{6}}{A_{3}} \delta_{H}\right)=-\frac{1}{2 \sqrt{-A_{3}}}\left(A_{1}+A_{7}+A_{8} \delta_{H}\right)+\mathcal{O}(\varepsilon) .
$$

Isolating $\delta_{H}$ in Equation (7.61) we have

$$
\delta_{H}=\frac{1}{\frac{A_{5} A_{6}}{A_{3}}-A_{8}}\left(A_{1}+A_{7}-\frac{A_{4} A_{6}}{A_{3}}\right)+\mathcal{O}(\varepsilon) .
$$

where a straightforward computation gives us

$$
\begin{aligned}
& A_{1}+A_{7}-\frac{A_{4} A_{6}}{A_{3}}=M(Z), \\
& \frac{A_{5} A_{6}}{A_{3}}-A_{8}=-N(Z, \tilde{Z}),
\end{aligned}
$$

where $M(Z)$ and $N(Z, \tilde{Z})$ are the constants computed in Proposition 3.5. From (7.63) and (7.64) it follows that

$$
\delta_{H}=-\frac{M(Z)}{N(Z, \tilde{Z})}+\mathcal{O}(\varepsilon),
$$

which coincides with the Hopf bifurcation value computed in Proposition 3.7 setting $\alpha=\delta \varepsilon$.

From now on we are going to compute the Canard value $\delta_{c}$. From Theorem 7.7 the Canard happens for

$$
\kappa_{\mathcal{C}}=-\left(\frac{\gamma_{1}+\gamma_{5}}{2}+\frac{A}{8}\right)+\mathcal{O}(\sqrt{\varepsilon})
$$


Using equations (7.54) to (7.58), we have the following expression for $A$

$$
A=-\gamma_{2}+3 \gamma_{3}-2 \gamma_{4}-2 \gamma_{5}=\frac{4}{\sqrt{-A_{3}}}\left(-\frac{A_{10} A_{3}}{A_{6}^{2}}+\frac{A_{9}}{A_{6}}-A_{1}\right) .
$$

Using the same argument as above, Theorem 7.7 implies that

$$
\begin{aligned}
-\frac{1}{2 \sqrt{-A_{3}}}\left(\frac{A_{4} A_{6}}{A_{3}}+\frac{A_{5} A_{6}}{A_{3}} \delta_{C}\right) & =-\frac{1}{2 \sqrt{-A_{3}}}\left(A_{1}+A_{7}+A_{8} \delta_{C}-\frac{A_{10} A_{3}}{A_{6}^{2}}+\frac{A_{9}}{A_{6}}-A_{1}\right) \\
& +\mathcal{O}(\sqrt{\varepsilon}) .
\end{aligned}
$$

Isolating $\delta_{c}$ and using the previous computations, we obtain

$$
\delta_{c}=-\frac{M(Z)}{N(Z, \tilde{Z})}+\bar{A}+\mathcal{O}(\sqrt{\varepsilon})
$$

with

$$
\bar{A}=-\frac{1}{N(Z, \tilde{Z})}\left(-\frac{A_{10} A_{3}}{A_{6}^{2}}+\frac{A_{9}}{A_{6}}-A_{1}\right) .
$$

Observe that, in this particular case, $-N(Z, \tilde{Z})>0$ then $\operatorname{sgn}(A)=\operatorname{sgn}(\bar{A})$. Therefore, $\delta_{c}>\delta_{H}$, if $A>0$ and $\delta_{c}<\delta_{H}$, if $A<0$.

Since $\alpha=\delta \varepsilon$, this proposition gives us the existence of a curve $\mathcal{C}$ given by

$$
\mathcal{C}=\left\{(\alpha, \varepsilon): \alpha(\varepsilon)=\delta_{C} \varepsilon+\mathcal{O}\left(\varepsilon^{\frac{3}{2}}\right)\right\}
$$

Observe that the $\delta_{H}$ obtained in Theorem 4.10 coincide with the value we had obtained in general in Proposition 3.7, if we set $\varphi(v)=v$. Moreover, a straightforward computation shows us that the $\delta_{C}$ given by this proposition also coincide with the value obtained in Proposition 4.6 for the Canard trajectory when $\varphi(v)=v$. 


\section{References}

[BST06] Claudio A. Buzzi, Paulo R. da Silva, and Marco A. Teixeira. "A singular approach to discontinous vector fields on the plane". In: Journal of Differential Equations 231 (2006). The geometry of differential equations and dynamical systems, pp. 633-655.

[Eck83] Wiktor Eckhaus. "Relaxation oscillations including a standard chase on French ducks". In: Asymptotic analysis, II. Vol. 985. Lecture Notes in Math. Springer, Berlin, 1983, pp. 449-494. DOI: 10 . 1007 / BFb0062381. URL: http : / / dx . doi . org/10 . 1007 / BFb0062381.

[Fil03] A. F. Filippov. "Differential equations with discontinuous right-hand sides, and differential inclusions". In: Nonlinear analysis and nonlinear differential equations (Russian). FizMatLit, Moscow, 2003, pp. 265-288.

[GST11] M. Guardia, T. M. Seara, and M. A. Teixeira. "Generic bifurcations of low codimension of planar Filippov systems". In: J. Differential Equations 250.4 (2011), pp. 1967-2023. ISSN: 0022-0396. DOI: $10.1016 / \mathrm{j} \cdot \mathrm{jde} .2010 .11 .016$. URL: http://dx.doi.org/10. $1016 / \mathrm{j} \cdot \mathrm{jde} .2010 .11 .016$

[HG83] P. HOLMES and J. GUCKENHEIMER. Nonlinear oscillations, dynamical systems, and bifurcations of vector fields. Vol. Applied mathematical sciences, v. 42.0387908196. Springer, New York, 1983.

[KH15a] K. Uldall Kristiansen and S. J. Hogan. "On the use of blowup to study regularizations of singularities of piecewise smooth dynamical systems in $\mathbb{R}^{3}$ ". In: SIAM J. Appl. Dyn. Syst. 14.1 (2015), pp. 382-422. ISSN: 1536-0040. DOI: 10.1137/140980995. URL: http: //dx.doi.org/10.1137/140980995.

[KH15b] K. Uldall Kristiansen and S. J. Hogan. "Regularizations of two-fold bifurcations in planar piecewise smooth systems using blowup". In: SIAM J. Appl. Dyn. Syst. 14.4 (2015), pp. 1731-1786. ISSN: 1536-0040. DOI: 10.1137/15M1009731. URL: http://dx.doi.org/ 10.1137/15M1009731.

[KRG03] Yu. A. Kuznetsov, S. Rinaldi, and A. Gragnani. "One-parameter bifurcations in planar Filippov systems". In: Internat. J. Bifur. Chaos Appl. Sci. Engrg. 13.8 (2003), pp. 21572188. ISSN: 0218-1274. DOI: 10.1142/S0218127403007874. URL: http://dx.doi.org/ $10.1142 / \mathrm{S} 0218127403007874$.

[KS01a] M. Krupa and P. Szmolyan. "Extending geometric singular perturbation theory to nonhyperbolic points - fold and canard points in two dimensions". In: SIAM J. Math. Anal. 33.2 (2001), 286-314 (electronic). ISSN: 0036-1410. DOI: 10.1137/S0036141099360919. URL: http://dx.doi.org/10.1137/S0036141099360919.

[KS01b] M. Krupa and P. Szmolyan. "Relaxation oscillation and canard explosion". In: J. Differential Equations 174.2 (2001), pp. 312-368. ISSN: 0022-0396. DOI: 10.1006/jdeq. 2000.3929. URL: http://dx.doi.org/10.1006/jdeq.2000.3929.

[Kue10] Christian Kuehn. "From first Lyapunov coefficients to maximal canards". In: Internat. J. Bifur. Chaos Appl. Sci. Engrg. 20.5 (2010), pp. 1467-1475. ISSN: 0218-1274. DOI: 10. 1142/S0218127410026617. URL: http://dx.doi.org/10.1142/S0218127410026617.

[Lar15] Juliana Fernandes Larrosa. "Generic bifurcation in planar Filippov systems". PhD thesis. Universidade Estadual de Campinas, 2015.

[MR80] E. F. Mishchenko and N. Kh. Rozov. Differential equations with small parameters and relaxation oscillations. Vol. 13. Mathematical Concepts and Methods in Science and Engineering. Translated from the Russian by F. M. C. Goodspeed. New York: Plenum Press, 1980, pp. x+228. ISBN: 0-306-39254-4. 
[RS14] Carles Bonet Revés and Tere M. Seara. "Regularization of sliding global bifurcations derived from the local fold singularity of Filippov systems". In: (Feb. 2014). eprint: 1402.5237. URL: http://arxiv.org/abs/1402.5237.

[ST98] J. Sotomayor and M. A. Teixeira. "Regularization of discontinuous vector fields". In: International Conference on Differential Equations (Lisboa, 1995). World Sci. Publ., River Edge, NJ, 1998, pp. 207-223.

[Tei81] Marco Antonio Teixeira. "Generic singularities of discontinuous vector fields". In: An. Acad. Brasil. Ciênc. 53.2 (1981), pp. 257-260. ISSN: 001-3765.

[Tes12] Gerald Teschl. Ordinary differential equations and dynamical systems. Vol. 140. American Mathematical Soc., 2012.

[TS12] Marco Antonio Teixeira and Paulo Ricardo da Silva. "Regularization and singular perturbation techniques for non-smooth systems". In: Phys. D 241.22 (2012), pp. 19481955. ISSN: 0167-2789. DOI: $10.1016 / \mathrm{j}$.physd.2011.06.022. URL: http://dx.doi . org $/ 10.1016 / j \cdot$ physd.2011.06.022.

[Wig03] Stephen Wiggins. Introduction to Applied Nonlinear Dynamical Systems and Chaos. 2nd. Springer, 2003. ISBN: 0387001778. 